\title{
Space Weather: Physics, Effects and Predictability
}

\author{
A. K. Singh $\cdot$ Devendraa Siingh $\cdot$ R. P. Singh
}

Received: 30 November 2009/ Accepted: 22 September 2010/Published online: 17 October 2010

(C) Springer Science+Business Media B.V. 2010

\begin{abstract}
The time varying conditions in the near-Earth space environment that may affect space-borne or ground-based technological systems and may endanger human health or life are referred to as space weather. Space weather effects arise from the dynamic and highly variable conditions in the geospace environment starting from explosive events on the Sun (solar flares), Coronal Mass Ejections near the Sun in the interplanetary medium, and various energetic effects in the magnetosphere-ionosphere-atmosphere system. As the utilization of space has become part of our everyday lives, and as our lives have become increasingly dependent on technological systems vulnerable to the space weather influences, the understanding and prediction of hazards posed by these active solar events have grown in importance. In this paper, we review the processes of the Sun-Earth interactions, the dynamic conditions within the magnetosphere, and the predictability of space weather effects on radio waves, satellites and ground-based technological systems today.
\end{abstract}

Keywords Solar wind - Magnetosphere - Sun-Earth interactions - Space weather · Radio communications · Predictability of space weather

\section{Introduction}

The dynamic and highly variable conditions in the geospace environment including those on the Sun, in the interplanetary medium and in the ionosphere-magnetosphere system to the ground are referred as space weather (Baker 1998; Singh and Singh 2003). Solar activity, especially Coronal Mass Ejections (CMEs), solar flares and energetic particles are the major

\footnotetext{
A. K. Singh $(\bowtie)$

Physics Department, University of Lucknow, Lucknow 226007, India

e-mail: aksphys@gmail.com

D. Siingh

Indian Institute of Tropical Meteorology, Pune 411-008, India

R. P. Singh

Space Physics Laboratory, Physics Department, Banaras Hindu University, Varanasi 221005, India
} 
factors controlling space weather. Space weather is greatly influenced by the speed and density of the solar wind and the interplanetary magnetic field (IMF) carried by the solar wind plasma. A variety of physical phenomena are associated with space weather, including geomagnetic storms, energization of the Van Allen radiation belts, geomagnetic activity, ionospheric disturbances and scintillations, aurorae, and geomagnetically induced currents at the Earth's surface. Recently, Lilensten and Belehaki (2009) have defined the term "space weather" as "the physical and phenomenological state of natural space environments. The associated discipline aims, through observation, monitoring, analysis and modeling, at understanding and predicting the state of the Sun, the interplanetary and planetary environments, and the solar and non-solar driven perturbations that affect them, and also forecasting and nowcasting the potential impacts on biological and technological systems".

The shock waves associated with CMEs are important drivers of space weather as they compress the magnetosphere and trigger geomagnetic storms (McAllister and Crooker 1997; Farrugia et al. 1997; Li et al. 2001; Cargill and Harra 2007; Cargill 2009). Solar energetic particles (SEPs), accelerated by the solar flares and the associated CME shocks, are also important aspects of space weather as they may damage electronics onboard spacecraft and may affect human health aboard satellites and aircraft (Valach et al. 2009).

The solar wind interacting with the Earth's magnetic field shapes the magnetosphere and provides a key link between the solar atmosphere and the Earth's environment. The energy transferred by the solar wind to the Earth's atmosphere is very much smaller than both the solar radiation energy and energies involved in the Earth's atmosphere; however, the near-Earth space environment reacts in a nonlinear way to the solar wind and may cause damage to a sophisticated "high tech" civilization.

Space weather research addresses physical processes in space ranging from solar phenomena to their impact on interplanetary space, geospace and at the surface of the Earth. Physical understanding of involved chain of processes is based on a combination of observation, data analysis and interpretation, theoretical and empirical modeling and numerical simulations. The important motivation behind space weather research is its impact on technological systems in space, in the air and on the ground. Operational models for nowcasting and forecasting space weather effects have been developed and updated from time to time (Forbes et al. 2006; Forsyth et al. 2006; Baker et al. 2007). Watermann et al. (2009a, b) have reviewed important models, which demonstrate the advance of our physical understanding of solar events as they evolve on their way from the Sun to the Earth.

The overall objective of this paper is to discuss the present understanding of the physics, the effects and the predictability of key drivers of space weather. The general scheme of the paper is as follows. In Sect. 2, we consider the physical nature of solar activity responsible for space weather such as CMEs, solar flares, energetic particles including galactic cosmic rays, and the solar wind. Their interactions and manifestations in the interplanetary space, magnetosphere and ionosphere are also discussed. Section 3 is focused on space weather effects and their relations to the space environment. Section 4 deals with the predictability of space weather. Finally, a summary of the paper is given in Sect. 5 .

\section{Physics of Space Weather}

The first step of understanding of the Sun-Earth connection was made by Edmund Halley following the spectacular auroral display in Europe in March 1716; he suggested that charged particles moving along the Earth's magnetic field lines were the cause of the 
aurora (Halley 1692). Following that, Celsius and Hiorter in 1747 discovered the temporal coincidence between compass needle variations and bright auroral displays (Celsius and Hiorter 1747). The geomagnetic activity connection to solar processes was established by the mid-nineteenth century. That active, bright auroras and geomagnetic disturbances followed a solar flare was found by Carrington in 1859; long term observations showed 11-year variability both in sunspot numbers and the occurrence frequency of magnetic disturbances and auroras (Carrington 1859).

The time scales interesting for studies of space weather processes are determined both by the intrinsic time scales of the processes themselves and also by the lead time over which predictions can be made. Solar and magnetospheric processes have several time scales, ranging from solar cycle ( $\sim 11$ and 22 years) and longer to 27 days (recurrent solar activity associated with the solar rotation period), days (magnetic storms), hours (magnetospheric substorms), and even minutes and seconds (charged particle acceleration events, plasma instability growth times). On the other hand, before an event can be predicted, some indication of its cause must already have been observed. Table 1 depicts the domain-oriented catalogue of the systems affected by space weather, their effects and their measurable parameters, in tabular form (Koskinen et al. 2001). Single Event Effect (SEE) refers to soft (nondestructive) or hard (destructive) damage caused to electronic equipment onboard a spacecraft or aircraft by high energy charged particles, which may be in the form of either or both an induced transient pulse causing a soft error in logic or support circuitry and a transient current leading to soft/hard damage to the power supply (Crosby et al. 2006). Electrostatic Discharge (ESD) refers to the spark on a satellite which occurs when its surfaces or interior elements become highly charged relative to the space plasma or to neighbouring satellite components.

\subsection{Sun and Solar Activity}

The Sun is the epicentre of space weather events. It affects the Earth and its environment in a variety of ways and on many different time scales. Space between the Sun and the planets is not empty as was contemplated up to the 1950s. It is filled by tenuous magnetised plasma, which is a mixture of ions and electrons flowing away from the Sun. In fact, the Sun's outer atmosphere is so hot that not even the Sun's gravity can prevent it from continuously evaporating. The escaping plasma carries the solar magnetic field along with it (Parker 1957), out to the boundary of the heliosphere where its dominance finally ends (Schwenn 2006a, b) in interstellar space. Solar activity includes phenomena related to solar flares and CMEs, and their recurrence in time and space.

Solar activity varies with the well known 11-year cycle, which introduces an 11-year activity cycle into geomagnetic records. As the orientation of the magnetic dipole axis relative to the Sun-Earth line changes over the course of the year, the activity is largest during the equinoxes when the dipole is tilted along the Earth's orbital track and the projection of the IMF to the geomagnetic field maximizes (Russell and McPherron 1973). Similarly the activity level is at minimum during the solstices when the projection of the IMF onto the Earth's field is on average a minimum. Figure 1 illustrates the close relationship of the long-term solar activity (characterized by the monthly mean of the sunspot number, shown in green) and the geomagnetic activity using the planetary magnetic $A_{P}$ index (shown in blue). The left panels show the long term solar cycle variability and geomagnetic activity for seven solar cycles. The top right panel illustrates how the geomagnetic activity maximizes during the declining phase of the solar cycle. 


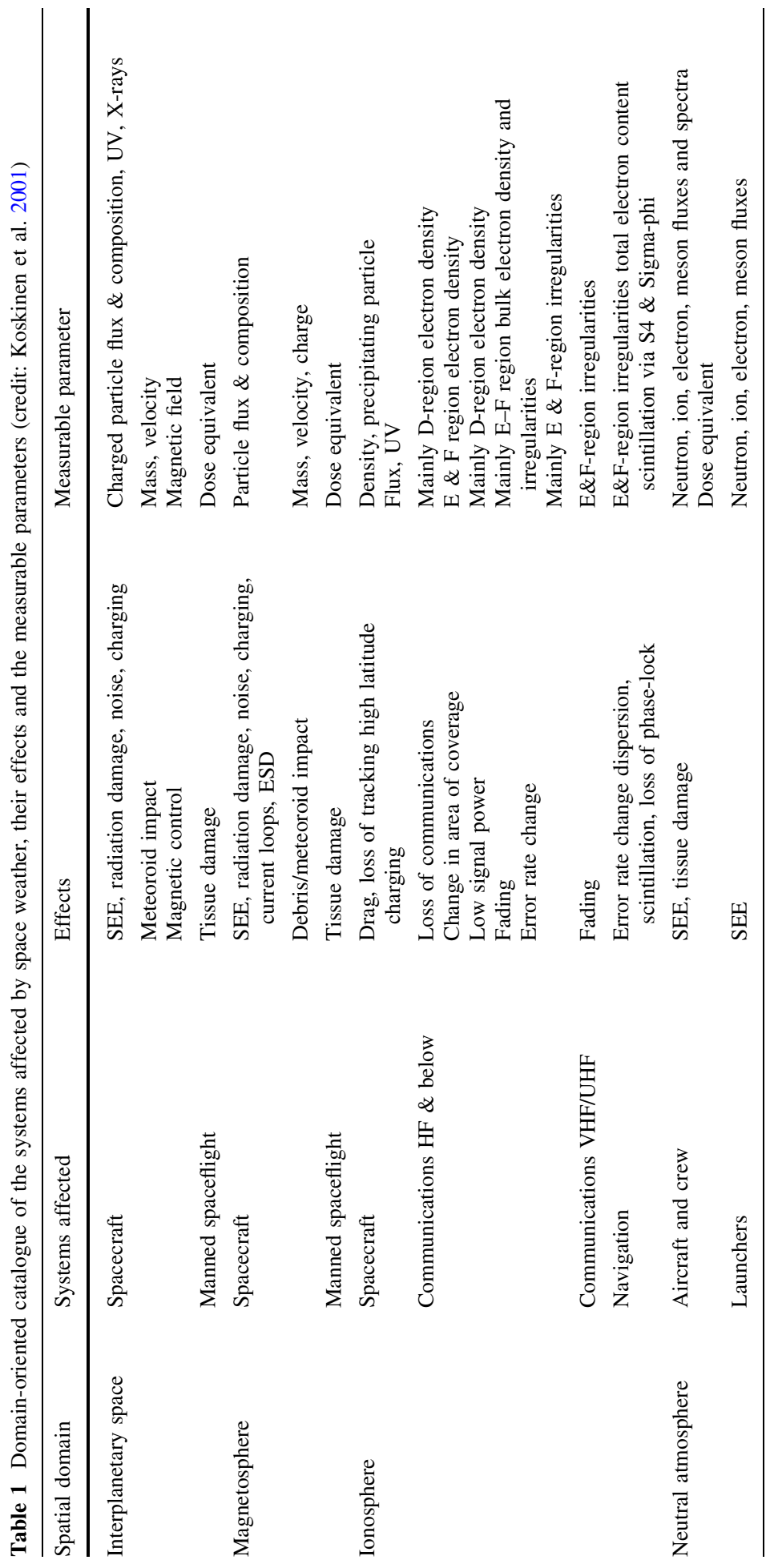




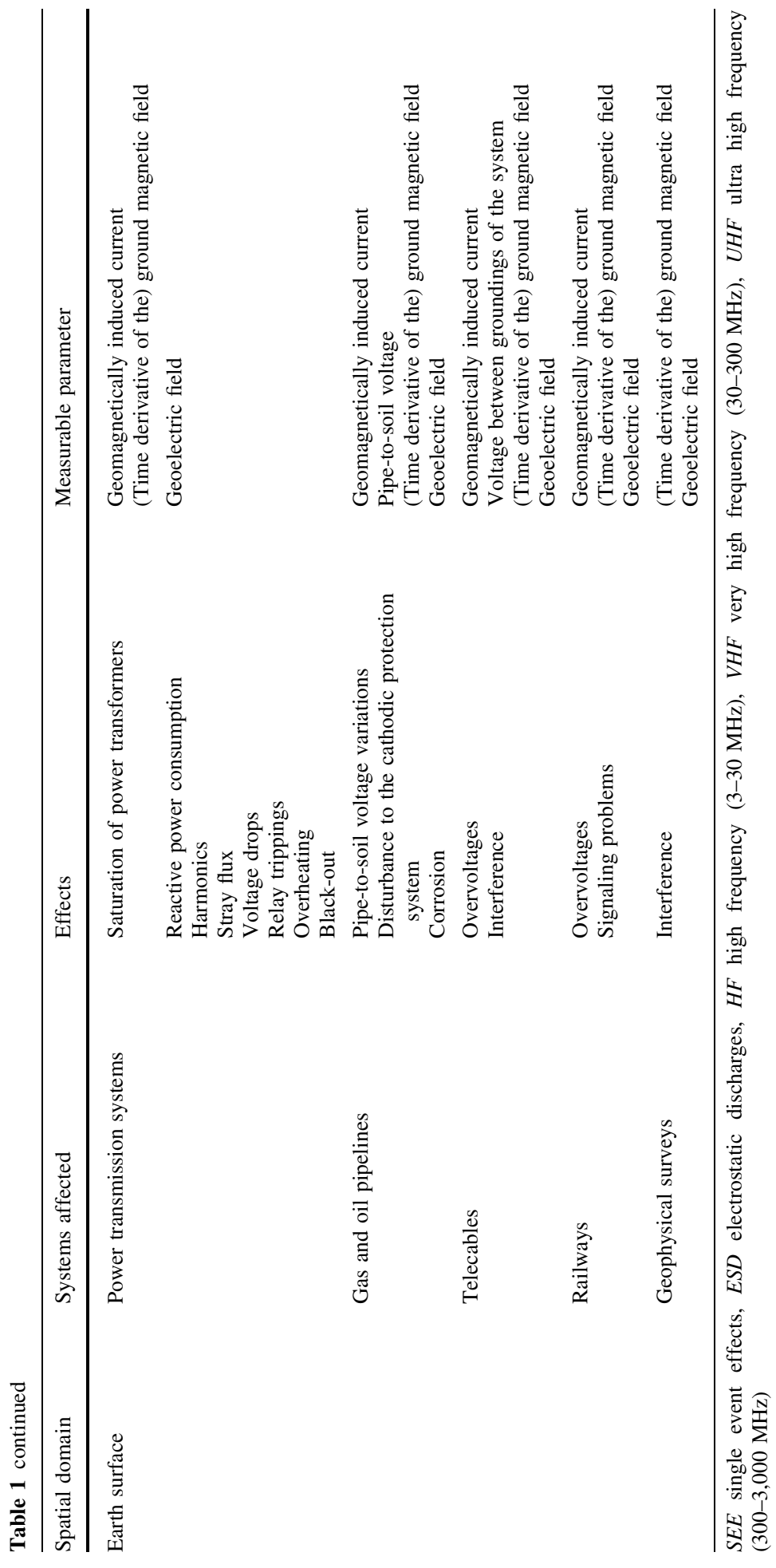



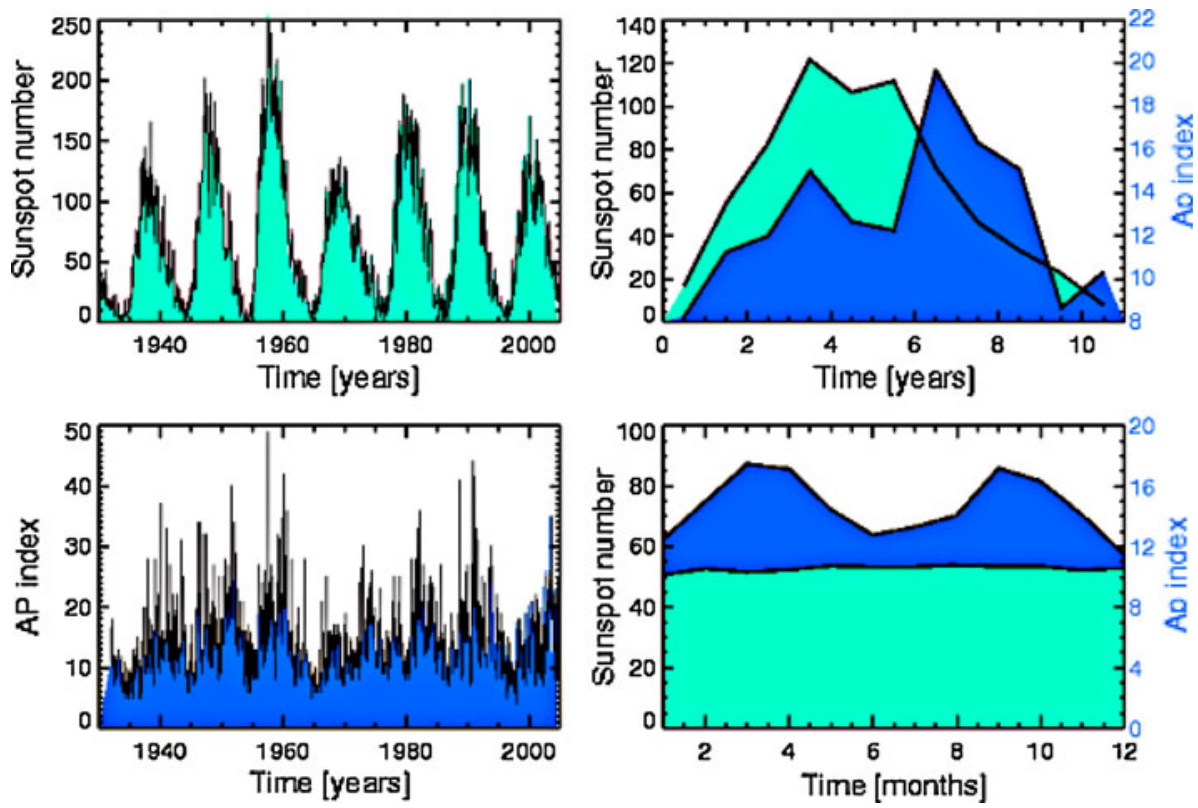

Fig. 1 Illustration of solar activity and geomagnetic activity (credit: Schwenn 2006b; http://www.ava. fmi.fi)

The bottom right panel shows the semiannual variations obtained by averaging the monthly values in the left hand plots (Schwenn 2006b).

\subsubsection{Coronal Mass Ejections (CMEs)}

Since Carrington's serendipitous solar flare observation in 1859 , it was established that geomagnetic storms were linked to solar activity. More than a 100 years later, a different category of highly energetic transient events on the Sun was discovered (Brückner 1974; Gosling et al. 1974), in the course of which huge amounts of gas are violently ejected from the Sun into interplanetary space. Such transients originate from the coronal region of the Sun and are commonly referred as coronal mass ejections (CMEs). They had not been observed earlier, because the corona is much less bright than the photosphere, and may only be directly observed during a solar eclipse. CMEs expel vast clouds of solar magnetic flux and plasma into interplanetary space and their pronounced significance for the Earth was soon revealed (Hildner 1977; Wagner 1984; Bastian et al. 1998, 2001; Gopalswamy 2004). The structure of a CME contains a leading density front followed by a dense core formed of solar prominence material and then a plasma void. Magnetic flux-rope structures are often embedded in the cavity.

CMEs are characterized by their speed, angular width, and central position angle in the sky plane. Their speeds are often fairly constant over the first few solar radii (St Cyr et al. 2000) and range between 300 and 3,000 $\mathrm{km} \mathrm{s}^{-1}$ (Bothmer and Zhukov 2006). CMEs have mass and kinetic energy in range $5 \times 10^{12}-5 \times 10^{13} \mathrm{~kg}$ and $10^{23}-10^{24} \mathrm{~J}$, respectively, and they have angular widths between $24^{\circ}-72^{\circ}$ (Bothmer and Zhukov 2006). The source regions of CMEs can be bipolar regions of the Sun's photospheric magnetic field, both active and quiescent (Cremades and Bothmer 2004; Tripathi et al. 2004). The onsets of 
large and fast CMEs are usually accompanied with flaring electromagnetic radiation at X-ray, EUV and sometimes even $\gamma$-ray wavelengths (Aschwanden 2004). The occurrence rate of CMEs vary with solar activity; during low solar activity it is less than one event per day and during high solar activity period it can exceed 10 or more (Bothmer and Zhukov 2006). CME observations by coronagraphs can be adequately described through magnetic flux-rope models (Low 2001; Thernisien et al. 2006).

The interplanetary manifestation of CMEs (termed ICMEs) propagates outward from the Sun, often at a velocity higher than the usual background velocity of the solar wind (Schwenn 2006a, b). If the velocity difference exceeds the solar wind acoustic velocity, the fast ICME builds up a plasma shock front (Bothmer and Schwenn. 1998) which, when hitting the Earth's magnetosphere, can trigger a geomagnetic storm. Figure 2a shows a CME event on February 27, 2000, observed by the Large Angle Spectroscopic Coronograph (LASCO C3 coronagraph) on the SOHO spacecraft (Schwenn et al. 2005). The instruments aboard SOHO have recorded more than $10^{3} \mathrm{CMEs}$ during the 23rd solar cycle (1996-2008) with unprecedented spatial and temporal resolutions; the observed CMEs are of various sizes, angular widths and speeds (Messerotti et al. 2009). Figure $2 b$ shows a schematic of the three dimensional structure of an ICME including the CME plasma, the magnetic cloud, an upstream shock, the signature of bidirectional suprathermal electrons (BDEs) and the interplanetary magnetic field (IMF) (Zurbuchen and Richardson 2006).

ICMEs that have enhanced slowly rotating magnetic fields $(>10 \mathrm{nT})$, low proton temperatures and low plasma $\beta$ (ratio of the thermal and magnetic field energies) are termed "magnetic clouds" (MCs). An important unsolved question about MCs is whether they have a root in the Sun or are disconnected from the solar surface (Akasofu 2007). Observations of BDEs fluxes favour the root hypothesis (Burlaga 1991) while the huge dimensions of MCs deduced from Ulysses measurements favour the disconnection hypothesis (Watermann et al. 2009a, b). Electrons having their origin at the Sun and flowing in opposite directions along magnetic field loops within ICMEs are called bidirectional electrons. BDEs are one of the widely-used signatures for identifying ICMEs (Gosling et al. 1987). CMEs mostly originate near the heliographic equator (St Cyr et al. 2000), except in a few cases which are seen at very high latitudes. These CMEs might have originated at mid-latitudes, but in projection appear poleward (Burkepile et al. 2004; Cremades and Bothmer 2004). Studies show that, during the solar minimum, CME occurrence peaks at the solar equator, but their source regions are centered in two belts on around $25^{\circ}$ North or South heliographic latitude (Cremades et al. 2006). This means that these CMEs must have been deflected towards the solar equator by the nearby coronal holes (Cremades et al. 2006). Coronal holes are the regions of low density solar corona, and they are broadly classified as polar, isolated or transient holes. The deflection of CMEs is proportional to the proximity and the size of nearby holes.

ICMEs are the primary cause of severe space weather at the Earth; hence their early detection and tracking from near the Sun to the Earth becomes important in forecasting geomagnetic storms. The Solar Mass Ejection Imager (SMEI), a sophisticated white-light imager (Eyles et al. 2003; Jackson et al. 2004) is currently operating on the US Coriolis satellite launched in January 2003 (Webb et al. 2006). Coriolis is a Sun synchronous satellite in a dawn-dusk circular polar orbit along the Earth's terminator at an altitude of $840 \mathrm{~km}$ and an inclination of $98^{\circ}$ relative to the equatorial plane. The SMEI has been obtaining images of the nearly full sky every $102 \mathrm{~min}$ (Webb et al. 2009). The white-light images of ICMEs contain a bright leading loop-like structure followed by a dark cavity and a bright core of denser material. Observations from SMEI show that ICMEs are directed towards the Earth with corresponding source regions on the visible solar disk. 
Fig. 2 a The coronal mass ejection on February 27, 2000, observed by the LASCO C3 coronograph. The Sun is drawn to scale. The image is a difference between two exposures taken about $10 \mathrm{~h}$ apart from each other (credit: Schwenn et al. 2005). b Schematic of the threedimensional structure of an ICME and upstream shock, relating magnetic field, plasma and bidirectional beams of suprathermal $(\geq 100 \mathrm{eV})$ electrons (BDEs) signature (credit: Zurbuchen and Richardson 2006)

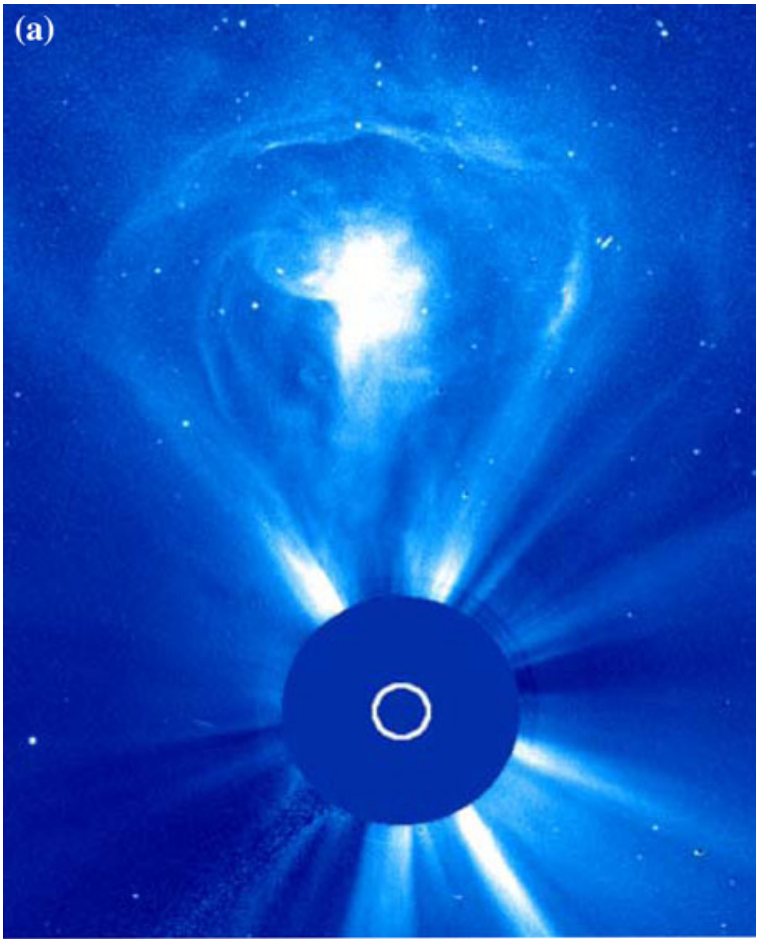

(b)

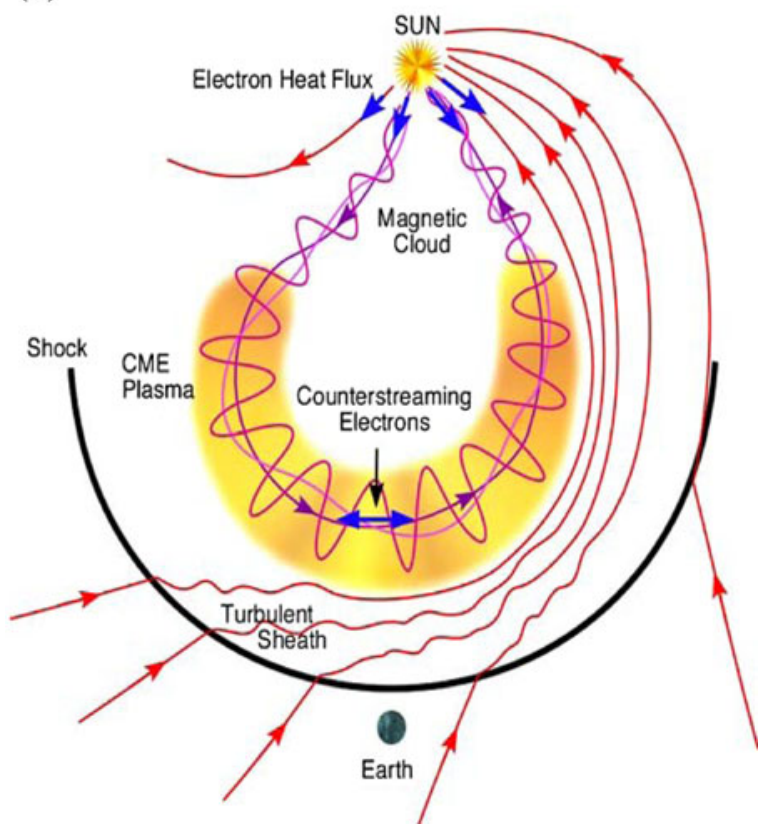


SMEI is less sensitive to CMEs erupting off the dark-side of the Sun because of Thomson scattering at these large elongations and frequent multiple CMEs can merge at large distances from the Sun. Direct observations of CMEs all along the Sun-Earth line from the lower corona to the Earth's magnetosphere are being carried out from the Sun-Earth Connection Coronal and Heliospheric Investigation (SECCHI) onboard the two NASA-STEREO spacecraft launched on October 25, 2006 (Howard et al. 2008; Kaiser et al. 2008; Davies et al. 2009).

The observations have provided new insights into the three dimensional structure of CMEs and their evolution in the heliosphere (Davis et al. 2009). The results analyzed have shown that the STEREO mission can give a warning of a CME impacting the magnetosphere at least $24 \mathrm{~h}$ in advance (Davis et al. 2009).

The Solar Dynamics Observatory (SDO) has been launched on February 11, 2010 into an inclined geosynchronous orbit to understand the causes of solar variability and its impacts on the Earth. The inclined orbit allows continuous observations of the Sun. The scientific experiments aboard are the Atmospheric Imaging Assembly (AIA), the EUV Variability Experiment (EVE) and the Helioseismic and Magnetic Imager (HMI). These three instruments observe the Sun simultaneously, make the required measurements to understand the mechanisms involved in the quasi-periodic solar cycle (11-year) activity and its relation with the Sun's EUV spectral irradiance, the state of active region magnetic flux topology before and after the occurrence of solar flares, CMEs and filament eruptions. These studies are aimed at making accurate and reliable forecasts of space weather to understand the mechanisms of the processes involved (http://sdo.gsfc.nasa.gov). Figure 3 shows one of the first images collected from SDO's AIA instrument (Klotz 2010), showing a CME filament launching from the Sun in the ultraviolet (false color). The SDO will spot eruptions as they occur, giving forecasters an approximately 4-days warning time. SDO images the Sun every $0.75 \mathrm{~s}$ and transmits about 1.5 terabytes of data per day at a resolution 10 times better than today's high resolution television (Klotz 2010).

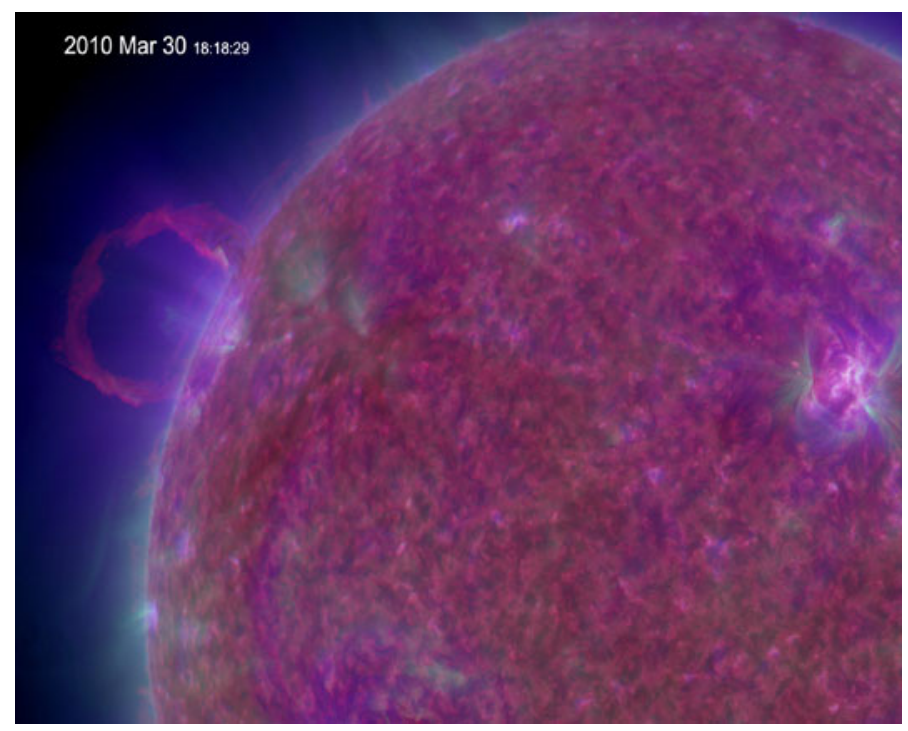

Fig. 3 One of the first images collected from the Solar Dynamics Observatory's Atmospheric Imaging Assembly (AIA) instrument was a close-up, false-color ultraviolet view of a coronal mass ejection filament launching from the Sun (credit: Klotz 2010) 
The current CME initiation models are based on magnetic flux rope structures being either present before the eruption takes place or created during the eruption as a consequence of magnetic reconnection (Roussev and Sokolov 2006; Forbes et al. 2006). They are based on the fact that (a) helical structures in images of erupting prominences and CMEs are observed, (b) correlations between ICMEs and MCs are observed, and (c) CMEs and erupting prominences possess some amount of twisted magnetic field. The initial flux rope is assumed to be suspended in the corona by a balance between magnetic pressure and tension forces. A highly twisted field is required to produce the eruption. Numerical simulation shows the evolution of a twisted magnetic flux-rope from below the photosphere into the corona (Amari et al. 2004; Fan and Gibson 2007). If a flux-rope does not exist prior to the eruption, then it is formed in the course of eruption by magnetic reconnection (Miki'c and Linker 1994; Jacobs et al. 2006). Shearing motions continuing for a long time energize the magnetic field and might lead to the formation of a flux-rope (Jacobs et al. 2006) by reconnecting the opposite polarity feet of a sheared magnetic arcade. In the case of a multipolar (complex) magnetic topology, the breakout model for CME eruption is used (DeVore and Antiochos 2008; Lynch et al. 2008), in which the eruption is triggered by magnetic reconnection taking place in the current sheet located above the sheared arcade. Observational studies of the CME source region show the presence of both bipolar and quadrapolar topology ( $\mathrm{Li}$ and Luhmann 2006; Ugarte-Urra et al. 2007), although bipolar topology is more common.

Numerical simulations (Priest et al. 1994; Priest and Forbes 2000, 2002) show that slow, intermediate and fast mode shocks should form ahead of CMEs with speeds of 200-300, 300-900 and greater than $900 \mathrm{~km} \mathrm{~s}^{-1}$, respectively. The existence of fast mode shocks in the solar corona is strongly supported by the observation of rapidly drifting (in frequency) solar radio bursts and their association with fast $\left(>400 \mathrm{~km} \mathrm{~s}^{-1}\right)$ CMEs (Gopalswamy 2004).

The coherent interplanetary magnetic field (IMF) structure, the strongly varying field and plasma density in the sheath region preceding the CME proper, the fast solar wind speed as well as the interplanetary shock itself are all effective drivers of geomagnetic activity (Farrugia et al. 1997). A strongly southward magnetic field inside the CME proper tends to drive high magnetospheric ring current activity. The more variable fields and densities in the sheath region sandwiched between ICMEs and associated shocks drive the strongest activity at high geomagnetic latitudes, the auroral regions (Huttunen and Koskinen 2004).

\subsubsection{Introduction to Solar Flares and Their Effects}

A solar flare can be defined as the sudden and explosive release of energy $\left(\sim 10^{19}-10^{25} \mathrm{~J}\right)$ from a localized active region of the Sun, mainly in the form of electromagnetic radiation across the entire spectrum. The heated plasma producing radiation resides in the closed loops created during the eruption process (Gopalswamy 2009). Solar flares and coronal mass ejections (CMEs) are the result of the sudden release of magnetic stresses accumulated for some period of time in the lower solar atmosphere (R'egnier and Priest 2007). The magnetic field created in the convection zone, having complex topology, ascends through the photosphere and emerges into the corona. The coronal field changes reflect the energy stored as a result of currents injected through the photosphere (Green et al. 2002; Schrijver 2007). The stressed magnetic fields appearing in the corona do not relax instantaneously into an unstressed state, but wait to relax until a specific condition has been met (Schrijver 2007). However, the required specific condition is not yet identified. 
It is not known exactly how the energy is released or how the complex magnetic configuration becomes unstable and the flare is triggered. Even it is unclear what happens after the energy is released and what fraction of energy goes into heating, particle acceleration, electromagnetic radiation, and mass motion, etc. It is also important to know the magnetic configuration in the pre-flare phase (Svestka 1976; Gosling 1993).

Based on topology, solar flares are classified as a compact or simple-loop flares and two- ribbon flares (Messerotti et al. 2009). Compact flares occur in single loops whose shape and volume do not change significantly during the flare and which do not emit charged particles. Flares that occur in magnetic arcades and show two regions of particle emission on both sides of a magnetic inversion line in the lower atmosphere are called two parallel flare/ribbon flares. The solar flare observed during the Halloween event of October and November 2003 was a parallel ribbon flare (Crosby et al. 2006).

Different kinds of radiation emanate from different parts of the flare and are released at different times of the flare process (Schwenn 2006a). The first visible signature of a flare appears in soft X-rays $(0.1-10 \mathrm{~nm})$ at energies up to a few tens of $\mathrm{keV}$ originating from the sudden heating of coronal plasma. A few minutes after the soft X-ray bursts, non-thermal hard X-rays with energies of tens of $\mathrm{keV}$ to a few $\mathrm{MeV}$ are observed in many, but not all, flares (Garcia 2004).

The intensity of soft X-rays in the $0.1-0.8 \mathrm{~nm}$ band as measured on the GOES spacecraft has been used for flare classification. Solar flares are classified as A, B, C, M or $\mathrm{X}$ according to the peak flux (in $\mathrm{Wm}^{-2}$ ). Each class has a peak flux ten times greater than the preceding one, with $\mathrm{X}$ class flares having a peak flux of order $10^{-4} \mathrm{Wm}^{-2}$. Within a class there is a linear scale from 1 to 9 . The X-ray flare of the Halloween storm of October 28, 2003 is shown in Fig. 4. The storm was classified as X17.2, corresponding to the measured power of $1.72 \mathrm{~mW} \mathrm{~m}^{-2}$ (Crosby et al. 2006; http://www.sec.noaa.gov/sxi/ index.html).

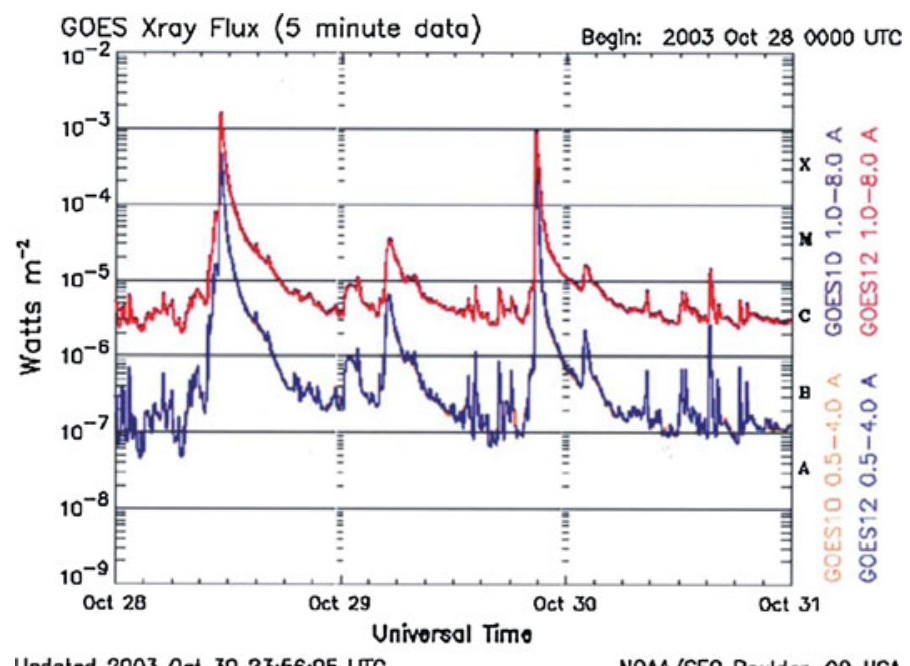

Fig. 4 Global solar X-ray flux observed from 1.5 to $12.4 \mathrm{keV}(1-8.0 \AA)$ and $3.1-24.8 \mathrm{keV}(0.5-4.0 \AA)$ for the time period 28-31 October 2003. The large X17.2 X-ray flare occurred on 28 October 2003 at 11:10 UT (credit: Crosby et al. 2006; http://www.sec.noaa.gov/Data/alldata.html) 
Apart from the soft X-rays, the extreme ultra violet EUV (10-125 nm) emissions are among the most geoeffective parameters of the solar electromagnetic spectrum. In the EUV range, the total flux density is a very substantial factor that changes the state of the Earth's ionosphere. The energy content of the other components of flare phenomena such as the solar protons and electrons, solar wind and CMEs are substantially lower (Avakyan 2008). The EUV emissions and visible light are used to explore the flare transition layer that is abruptly heated up (Noyes and Withbore 1972). The Lyman- $\alpha$ line (121.6 nm) and the other members of the Lyman series of hydrogen, namely Lyman- $\beta$ and Lyman- $\gamma$ (102.6 and $97.2 \mathrm{~nm}$ ), come from the upper chromosphere, whereas the Balmer series of hydrogen $(\mathrm{H} \alpha, 656.3 \mathrm{~nm})$ becomes dominant from lower in the solar atmosphere (Rottman 1999). Protons accelerated during the flare process can penetrate deep into the solar atmosphere and may excite gamma rays and white-light emissions. The required proton energy is larger than $25 \mathrm{keV}$. The same energetic electron population in the presence of strong chromospheric magnetic fields radiates microwave bursts through the process of synchrotron radiation. Very good time correlation between the hard X-ray bursts and impulsive microwave radiation (frequency $>1 \mathrm{GHz}$ ) has been observed (Pick et al. 1990; Holman 2003). The observed outward moving type IV radio bursts (frequency $<200 \mathrm{MHz}$ ) have been considered to be caused by electrons trapped in a closed magnetic cloud ejected from the flare site (Kahler 1992). However, this interpretation is still under debate.

The flare accelerated electrons and protons are carried out into the heliosphere and they are accompanied by radio wave emissions in the frequency range from a few $\mathrm{kHz}$ to $\mathrm{MHz}$, known as type III radio bursts. Many strong solar flares are also accompanied by type II radio bursts $(20 \mathrm{MHz}<$ frequency $<$ several hundred $\mathrm{MHz}$ ) (Cane and Erickson 2005). Kilometric type II radio bursts have also been observed, but there is controversy about their origin. Cliver et al $(1999,2004)$ argue that both the metric type II and kilometric type II bursts are produced by CME-driven shocks, whereas many others consider them to be produced by flare-driven shocks (Gopalswamy et al. 1998; Cane and Erickson 2005).

The various types of radiation produced during the solar flare process may substantially change the radiation environment of the Earth. The EUV radiation (in particular the Lyman- $\alpha$ radiation of hydrogen) is absorbed in the Earth's atmosphere and causes its instantaneous heating. The consequent expansion may lead to sudden increase of the drag of Earth-orbiting satellites and thereby lower their orbits (Lean 1989, 1991). The Halloween storm on October 28, 2003 had spectacular enhancements of solar X-rays (flares), solar energetic particles and there was a large geomagnetic storm (Lopez et al. 2004). Baker et al. (2004) showed that the period of October-November 2003 was a period of exceptionally high radiation belt variability and that the plasmasphere was reduced to less than half its normal size. The presence of X-rays and gamma radiation may damage our technological systems in space, and may harm the health of humans in space.

The efforts to forecast solar flares are usually based on different conceptual procedures (Jensen et al. 2004; Barnes et al. 2007; Falconer et al. 2007; Georgoulis and Rust 2007; Contarino et al. 2009; Colak and Qahwaji 2009). Messerotti et al (2009) have briefly reviewed the forecasting methods. The recently developed Automated Solar Activity Prediction (ASAP) is an upgrade of the prediction system of Qahwaji and Colak (2007) which is based on machine learning and sunspot measurements. ASAP is a hybrid system composed of two neural networks, which provide both the flaring probability of each sunspot group and the relevant flare intensity. Sunspot data are used from the SOHO/MDI Continuum and Magnetogram images. The success rate of prediction of a flare is $\sim 70 \%$. 


\subsubsection{Solar Radio Emissions}

Solar radio noise and bursts were discovered during the early research on radar at the time of the Second World War (Southworth 1945; Hey 1946). Solar radio bursts produced unexpected jamming of this new technology that was then under rapid development. Solar radio bursts associated with solar activity can have much larger intensities than normal solar radio emissions, and cause considerable excess noise in a wireless cell site that might be immersed in it (Barron et al. 1985). The noise produced by a radio burst depends on its frequency. Lanzerotti et al. (2005) showed that radio bursts with frequency $\sim 1 \mathrm{GHz}$ and amplitude $\sim 10^{3} \mathrm{SFU}$ (Solar Flux Unit, $1 \mathrm{SFU}=10^{-22} \mathrm{~W} / \mathrm{m}^{2}$ ) produce a noise power $>3 \mathrm{~dB}$. Thus a solar radio burst could disrupt wireless base stations that were pointed in the solar direction at the time. Lanzerotti et al. (2005) have pointed out that, statistically, a solar radio burst $\left(f \sim 1 \mathrm{GHz}\right.$ and greater) with amplitude $>10^{3}$ SFU may occur every few days during solar maximum conditions. The duration of bursty microstructures can also affect wireless base stations (Lanzerotti et al. 2005). Short term variations often occur within solar radio bursts; they may be many tens of $\mathrm{dB}$ larger than the underlying solar burst intensities upon which they are superimposed (Isliker and Benz 1994). The subject matter of solar radio bursts acting as direct sources of space weather effects by interfering with wireless systems have been widely studied (Goodman 2005; Gary et al. 2005; Lanzerotti 2007; Messerotti 2008).

Solar radio bursts can cause the loss of signal lock in a Global Positioning System (GPS) receiver and may lead to positioning and navigation failures (Klobuchar et al. 1999; Chen et al. 2005; Cerruti et al. 2006). Afraimovich et al. (2007, 2008) investigated the failure of the GPS for more than 10 min during a solar flare on December 6, 2006 during solar minimum and showed that the associated solar radio burst has a flux density of $10^{5}$ SFU, with a peak flux density of $10^{6}$ SFU. Kennewell (1989) pointed out the effect of solar radio bursts on downlink satellite communications. Because geostationary satellites are aligned with the Sun at the equinoxes, satellite communications can be disrupted by the increase of radio noise caused by solar radio bursts (Messerotti et al. 2009). Solar radio bursts exhibit a wide range of spectral shapes and intensity levels, knowledge of which is essential for determining the nature and severity of bursts on specific technologies such as radar, satellite-to-ground communications, or civilian wireless communications (Burton et al. 1975). Research on solar radio phenomena remains an active and productive field (Daly et al. 1996; Gary and Keller 2004; Mann 2005) because they provide relevant information on the source processes of solar weather. The applications of solar radio physics to space weather are key ones.

\subsubsection{Energetic Particles}

The radiation environment of the Earth's atmosphere is very dynamic and consists of several components of ionizing radiation: (a) galactic cosmic rays (GCRs); (b) solar energetic particles (SEPs); and (c) radiation belt particles. The origin, flux density, energy distribution, spatial and temporal variations of each of these particle populations significantly differ from each other. The energy of these particles ranges from a few keV to some $\mathrm{GeV}$. The fastest SEPs move with a velocity of more than half the speed of light, and they arrive at the Earth a few minutes after the light flash due to the solar flare (Svestka and Simon 1976; Tascione 1994; Vainio et al. 2009).

Galactic cosmic rays reach their maximum intensity when the Sun is least active and are at a minimum intensity during solar maximum. In contrast, during maximum solar activity 
an increased number of CMEs and solar flares produce high energy solar particles (O'Sullivan 2007). The Van Allen radiation belt contains electrons and protons with energies from several $\mathrm{keV}$ up to several hundreds of $\mathrm{MeV}$ (Walt 1994); their distribution in time and space depends on solar wind and other parameters. Thus, energetic charged particles (GCRs, SEPs and radiation belt) in the vicinity of the Earth vary with altitude, latitude and stage of solar cycle (Vainio et al. 2009).

The highest energy particles can even penetrate the skins of space probes inside and outside the Earth's magnetosphere, and damage spacecraft subsystems and scientific instrumentation in the payload (McKenna-Lawlor 2008). Communications with spacecraft can also be affected due to plasma induced surface changing and to energetic charged particle induced internal charging.

2.1.4.1 Galactic Cosmic Rays The primary particles in cosmic rays (Primary Cosmic Rays) are mostly protons from distant astrophysical objects. The energy range of cosmic ray (CR) primaries is from $\sim 1 \mathrm{GeV}$ upto $\sim 10^{20} \mathrm{eV}$ or more (Kudela 2009). The differential flux of primary cosmic rays varies between $10^{-28}$ and $10^{4}\left(\mathrm{~m}^{2} \mathrm{st} \mathrm{s} \mathrm{GeV}\right)^{-1}$ for the energy range $10^{20}-10^{8} \mathrm{eV}$ and it can be approximated by a power law decrease (Cronin et al. 1997). Cosmic rays of up to $\sim 10^{15} \mathrm{eV}$ are considered to be of galactic origin (Gaisser 1990). Studies indicate that supernova remnants are galactic CR accelerators up to energies close to the first knee $\left(\sim 3 \times 10^{15} \mathrm{eV}\right)$ in the energy spectrum of CRs (Funk 2008). The secondary cosmic rays (air showers) are produced in the Earth's atmosphere by the interaction of a primary cosmic ray with an atom of the atmospheric gas (Amsler et al. 2008).

The Cosmic Ray Isotope Spectrometer (CRIS) instrument on the ACE (Advanced Composition Explorer) spacecraft measures in interplanetary space the CRs in the energy range from $\sim 50$ to $\sim 500 \mathrm{MeV} /$ nucleon, with isotopic resolution for elements with $Z=2$ to $Z=30$ (Stone et al. 1998). George et al. (2009) compared the composition of galactic cosmic rays with the solar system constituents and reported similar relative abundances except at $Z=3-5,9-10$, and 19-25. The mass composition of CRs at ultra high energy is still under debate (Kudela 2009) and precise measurements of the CR composition in the energy interval $10^{16} \mathrm{eV}<E<10^{19} \mathrm{eV}$ is required to determine the transition from galactic to extra-galactic CR components.

The galactic cosmic rays (GCRs) enter the outer heliosphere (the region of the space where the Sun's magnetic field dominates) where the particles interact with the ambient solar wind plasma and the interplanetary magnetic field (IMF). The motions of these very energetic charged particles are there controlled by the IMF and by magnetospheric fields in the vicinity of the planets. The IMF in the heliosphere modulates the GCR flux at energies below a few hundred $\mathrm{MeV}$ per nucleon. The solar energetic particle population is modified by acceleration at the Sun and by plasma discontinuities in interplanetary space (Crosby 2007; Klecker 2009). Thus the GCR flux in the near-Earth space environment is controlled by solar magnetic activity and follows an 11 year cycle. The GCR activity is anti-correlated with the main solar activity indices (such as sunspot number) (McCracken et al. 2004). The GCR flux also responds to solar wind variation on both long and short time scales (Kudela 2009; Siingh and Singh 2010). In the heliosphere, plasma turbulence is not well characterized and hence the transport of charged particles parallel and perpendicular to the mean magnetic fields are not well understood. Even the form of mean magnetic field in the heliosphere is not well known (Fahr et al. 1986). To address these problems a large 
set of in situ measurements from large distances (up to several hundreds of AU) and with good coverage of heliolatitudes and longitudes is needed.

The CR time profile observed at the Earth's surface depends on many effects of interplanetary, geomagnetic and atmospheric origin. The routine monitoring of CRs using an ionization chamber started in January 1932 at Hafelekar, Austria, and CR intensity decreases during some geomagnetic storms by $\sim 1 \%$ was reported (Messerschmidt 1933). This effect was called a Forbush decrease (FD). Two types of FDs are observed; the nonrecurrent type is associated with CMEs and the $\sim 27$ day recurrent type is associated with corotating high speed solar wind streams (Lockwood 1971; Iucci et al. 1979). The relation between geomagnetic activity and FDs is not one-to-one (Kudela and Brenkus 2004). Verma et al. (2009) showed that all large FDs are associated with CMEs, the majority of them are "halo" CMEs coming directly towards the Earth and that most FDs are associated with interplanetary shocks. However, the correct descriptions of the effect of individual CMEs on cosmic ray modulation and on cosmic ray fluxes varying on short time scales are not known.

The Earth's magnetosphere acts as a shield against GCRs and SEPs (the latter are also referred to as solar cosmic rays). The transmission of CRs through the magnetosphere is quantified by the geomagnetic cut-off rigidity, which represents the lowest rigidity. The rigidity of a charged particle is defined as its momentum per unit charge. All particles with the same rigidity follow a track with the same curvature in a given magnetic field. This means that for each point in the magnetosphere and each direction from that point, there exist rigidity thresholds below which the cosmic rays are not able to reach this point. The threshold value is called the geomagnetic cut-off rigidity (Cooke et al. 1991). The cut-off rigidity also depends on whether a geomagnetic storm is in progress (Tyasto et al. 2008; Kudela et al. 2008; Desorgher et al. 2009). Desorgher et al. (2009) obtained magnetic field models during major magnetic storms using neutron monitor data and they also studied the effect of individual magnetospheric currents on variations in cut-off rigidity.

Cosmic rays produce ionization in the lower atmosphere which increases with increasing latitude and which decreases with increasing solar activity. Reliable quantitative models of CR ionization are based on an analytical approximation of the atmospheric cascade (O'Brien 2005) and on a full Monte Carlo simulation of the atmospheric cascade, including all the secondaries (Desorgher et al. 2005; Velinov and Mishev 2007). The additional ionization produced by GCRs enhances the conductivity of the lower atmosphere and hence may enhance the thunderstorm current which charges the global atmospheric electric circuit by facilitating enhanced charge transfer between the thunderstorms to the Earth (Singh et al. 2004; Siingh et al. 2007; Siingh and Singh 2010).

GCRs affect global electric circuit (GEC). Rycroft et al. (2007) discussed a GEC model driven by thunderstorms and electrified shower clouds and showed that each contributed almost equally to maintaining the ionospheric potential. Recently, Rycroft and Odzimek (2010) have discussed the effects of lightning and sprites on the ionospheric potential, and threshold effects on sprite initiation, obtained using an analog model of the global atmospheric electric circuit. The effects of GCRs on aerosol, lightning, ozone, cloud coverage and global temperature have been reviewed by Siingh and Singh (2010). Finally, it is suggested that a model of the GEC, including stratospheric aerosols, cloud cover, upward lightning, cosmic rays and the solar wind as inputs, needs to be developed.

There are two ways that GCRs can affect space weather systems: (a) the high energy charged particles produce direct changes in materials/systems exposed to them both in space and above the surface of the Earth, (b) the anisotropic distribution of GCRs incident on the Earth's atmosphere affects atmospheric processes differently, which manifest 
themselves as latitudinal/longitudinal dependencies. Cosmic ray anisotropies may also be used as a tool for remote sensing of magnetic field changes in the heliosphere (Kudela et al. 2000).

Galactic cosmic rays having energies greater than $500 \mathrm{MeV}$ can be used for short term alert systems for major geomagnetic storms. This is based on the fact that the Larmor radii of GCRs in the interplanetary medium are sufficiently large that they are affected by large scale interplanetary magnetic field inhomogenities originating at the Sun well before the disturbances reach the Earth's orbit and geomagnetic disturbances occur (Marusek 2007; Siingh and Singh 2010).

2.1.4.2 Solar Energetic Particles The significant sources of Solar Energetic Particle (SEPs) fluxes in the interplanetary medium are solar flares and shock waves driven by CMEs. The typical distribution of energy in a solar flare is about half for the plasma, one quarter for electromagnetic radiation and one quarter for high energy charged particles (Kudela 2009). Before 1990, it was presumed that SEP events were mostly driven by solar flares. Based on observational results in the early 1990s, it was realized that large SEP events are driven by CME shock waves rather than by solar flares (Reames et al. 1996 and references therein). The impulsive SEP events are attributed to solar flares, whereas the large gradual SEP events result from the continuous acceleration of particles at CME driven shocks (Gosling et al. 2005). The terms impulsive and gradual are applied to distinguish the time scales of SEPs (Reames 1999). The gradual events are due to erupting filaments as a part of a CME and are dominated by protons. Density structures of the extended corona and pre-existing energetic particles influence the production of SEPs (Gopalswamy et al. 2004; Kahler and Vourlidas 2005).

An example of SEP events during the 2003 Halloween events is shown in Fig. 5. The intensities of electrons and protons as measured by the GOES/SAMPEX/ACE satellites in several energy bands are shown for the period from October 26 to November 7, 2003 (Mewaldt et al. 2005; Schwenn 2006b). The major five SEP events along with X-ray flares (indicated by dotted vertical lines) are shown. The largest SEP event attained its peak intensity during shock passage of the Earth on October 29. It is associated with the X17.2 flare and a halo CME about a day earlier (Schwenn 2006b). The maximum proton energy was probably larger than $1 \mathrm{GeV}$. Energetic protons penetrated the SOHO spacecraft and caused the temporary malfunction of several CCD cameras (Schwenn 2006b).

Figure 6 shows the times of occurrence of high energy $(>30 \mathrm{MeV})$ proton events with large fluences $\left(>10^{9}\right.$ proton $\mathrm{cm}^{-2}$ ) during the period 1855-2001 (McCracken et al. 2001; Cliver and Svalgaard 2004). The events during the period 1855-1950 were obtained using the nitrate composition in ice-cores (McCracken et al. 2001). Ionospheric and satellite data were used to extend the list up to 2001. From the figure it is seen that the biggest event of 1859 had a fluence of $18.8 \times 10^{9}$ proton $\mathrm{cm}^{-2}$, whereas the next largest event occurred in 1895 with a fluence of $11.1 \times 10^{9}$ proton $\mathrm{cm}^{-2}$. The third largest event occurred in November 1960, with a fluence $\sim 9.7 \times 10^{9}$ proton $\mathrm{cm}^{-2}$.

After acceleration at flare sites and shock waves in the corona and in interplanetary space, the energy of protons in SEPs reaches from a few $\mathrm{keV}$ to $\mathrm{GeV}$. The observation of the peak intensities at low energies within a Corotating Interaction Region (CIR) at places that are not magnetically connected to the shocks (Richardson and Zwickl 1984) and no observation of a turnover of the spectra below a few tens of $\mathrm{keV} /$ nucleon suggest that the ions are locally accelerated either by a stochastic (Schwadron et al. 1996) or a compressional 

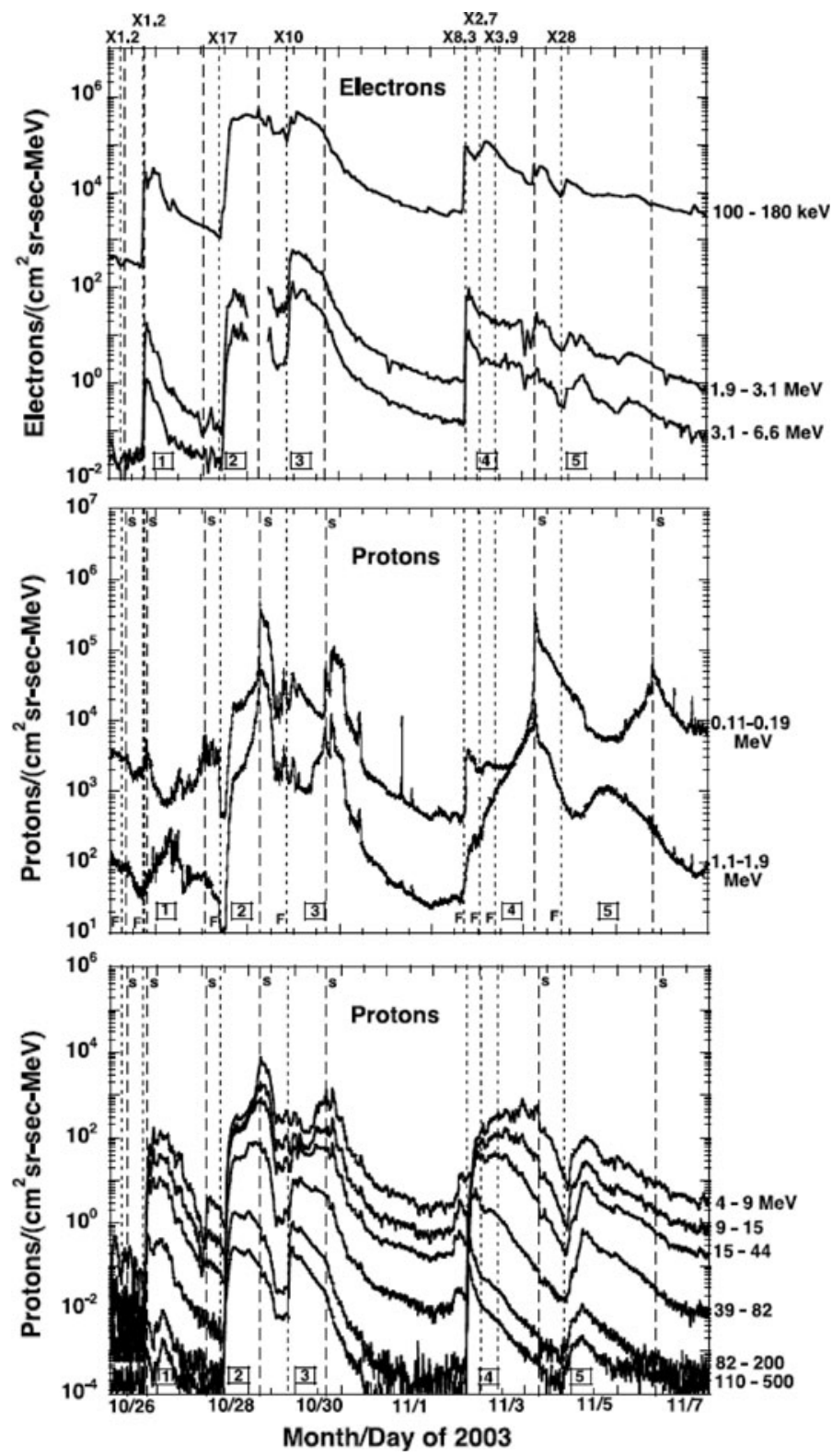

Fig. 5 Time history of energetic protons and electrons during the period from October 26, 2003 to November 7, 2003. The top panel shows electron data from EPAM/ACE (top trace) and PET/SAMPEX $(1.9-6.6 \mathrm{MeV})$. The SAMPEX points are averaged over separate polar passes, including only data obtained at invariant latitudes $>75^{\circ}$. It is possible that the intensities shown near the end of 29 October through 30 October (SEP event 3) are overestimated because of background contributions. The middle panel shows low-energy proton data from ACE/EPAM and the bottom panel includes protons measured by GOES-11 in six different energy intervals. The occurrence of X-class flares (obtained from NOAA) are indicated by dotted vertical lines with the intensity labeled above each line. Interplanetary shocks are indicated by dashed vertical lines labeled by an "s". Major proton events during this interval are labeled 1 to 5 (credit: Mewaldt et al. 2005; Schwenn 2006b) 
Fig. 6 The times of occurrence of all $>30 \mathrm{MeV}$ proton events with fluences exceeding $10^{9}$ proton $\mathrm{cm}^{-2}$ between 1855 and 2001. Pre-1950 values are based on nitrate composition in ice cores and later values are based on ionospheric measurements and direct satellite observations (credit: McCracken et al. 2001; Cliver and Svalgaard 2004)

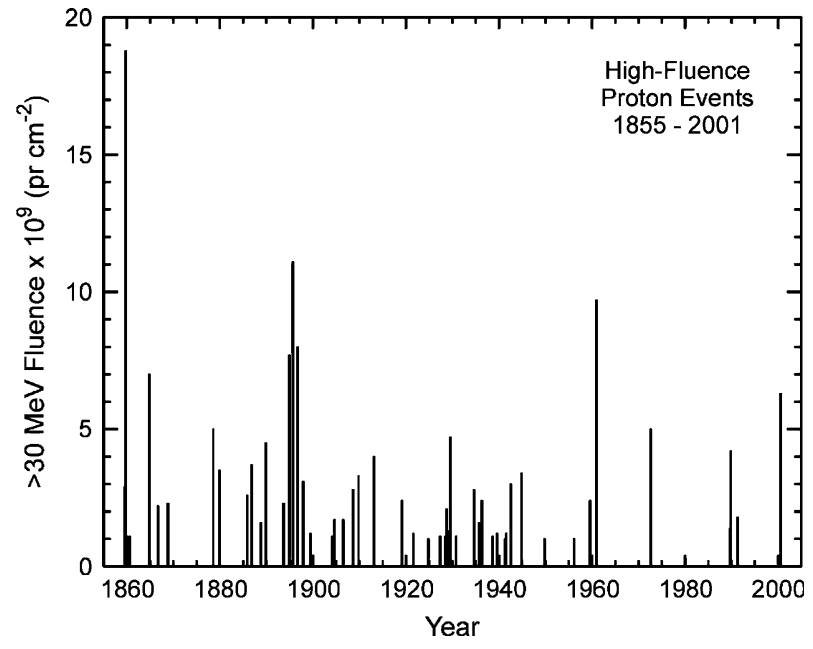

(Giacalone et al. 2002) mechanism. Recently, Shih et al. (2009) and Krucker et al. (2009) have discussed charged particle acceleration in solar flares.

The impulsive SEP events often have major enhancements in ${ }^{3} \mathrm{He} /{ }^{4} \mathrm{He}$ and enhanced heavy ion abundances because of resonant wave-particle interactions at the flare site (Balasubrahmanyan and Serlemitsos 1974). The results from several space missions have also shown enhancements of ${ }^{3} \mathrm{He}$ in the particle population accelerated at interplanetary shocks (Klecker 2009). Heavy ions are also observed during large events at high energies, and high charge states of Fe are observed in gradual events (Ryan et al. 2000). These anomalies may contain information about the acceleration and transport processes, and they require further studies.

SEP events also contain electrons and neutrons (Lin 1980). The energy spectra of electrons extends from a few $\mathrm{keV}$ to $100 \mathrm{MeV}$ (Moses et al. 1989). The energy spectra are usually represented by a power law spectrum with indices of 3 and higher (Lin et al. 1982). At low energies below a few tens of $\mathrm{keV}$, the fluxes are variable and show several spectral breaks and kinks (Lin 1985). Evenson et al. (1984) showed that most events with high fluxes of 5-50 MeV electrons appear to have been produced by flares which also produced observable fluxes of gamma-rays. In some cases electron fluxes with energies above $30 \mathrm{keV}$ are observed with a time delay of $30 \mathrm{~min}$ (Klassen et al. 2002). There is controversy about their origin (Haggerty and Roelof 2002). Klassen et al. (2005) analyzed the October 28, 2003, flare and reported three separate stages of injection. They remarked that "The association of two delayed injections with solar events is not well understood". Thus, the origin, acceleration and motion of electrons along and across the magnetic field need further investigation.

Biermann et al. (1951) suggested the possibility that solar flare accelerated protons with energies greater than $100 \mathrm{MeV}$ can interact with other atoms of the solar atmosphere and may cause nuclear reactions that release gamma-rays and relativistic neutrons. Chupp et al. $(1982,1987)$ reported the first direct detection of gamma-rays and neutrons on the NASA Seventh Orbiting Solar Observatory (OSO-7) following an impulsive solar flare. The released neutrons and gamma ray lines are related. The neutron spectra depend on the parameters of proton spectra and acceleration mechanism of protons. Hence, the observation of gamma ray lines can be used to study acceleration mechanism of protons and 
proton spectra. Even the abundance of elements can be studied. For example, the capture on protons produces the $2.223 \mathrm{MeV}$ gamma ray lines. The capture on ${ }^{3} \mathrm{He}$ gives no detectable radiation but it affects the time profile of the $2.223 \mathrm{MeV}$ line and can set constraints on the ${ }^{3} \mathrm{He} / \mathrm{H}$ ratio in the photosphere (Ramaty et al. 1983; Chupp 1988; Ramaty et al. 1996).

Relativistic solar neutrons reach the Earth unhindered and these are also received by ground-based neutron monitors. However, it is difficult to differentiate them from those neutrons that are generated from the interaction of relativistic SEP fluxes with the Earth's atmosphere. The enhanced neutron fluxes reaching the ground, also called ground level events (GLEs), are observed by a network of neutron monitors installed all over the globe (Bieber et al. 2004). During the 2003 Halloween events three GLEs were observed (Bieber et al. 2005). Vashenyuk et al. (2009) analyzed 32 large GLEs observed during the period 1956-2006 using data from the world wide network of neutron monitors. The analysis revealed two distinct relativistic proton populations: the early pulse like intensity increase with an exponential energy spectrum, and the late gradual increase with a softer energy spectrum of power law form. The component with the power law spectrum agrees with the time of maximum flux obtained from direct solar proton measurements on spacecraft and balloons (Vashenyuk et al. 2009).

Models based on evolving shock properties, wave-particle interactions and particle transport processes have been developed (Lee 2005; Vainio and Laitinen 2007, 2008; Ng and Reames 2008; Vainio et al. 2009) which could reproduce some features of shock associated SEP events. Characteristic features such as the start time of the event, peak flux, fluence and the corresponding energy spectrum, etc., are not predicted. This is due to the complexity of the physical processes involved in the generation, acceleration and transport of SEPs in space (Laurenza et al. 2009). The analysis and interpretation of data from the SDO and many other spacecraft will improve the situation.

2.1.4.3 Earth's Radiation Belts The Earth's radiation belts, commonly known as the Van Allen belts, are composed mainly of electrons and protons with energies greater than several tens of $\mathrm{keV}$ and up to several hundreds of MeV (Van Allen et al. 1958; Van Allen and Frank 1959). There are two radiation belts, the inner and outer belts. Charged particles in the radiation belts are trapped by the geomagnetic field, which is assumed to be dipolar. High energy particles are locally accelerated by magnetospheric processes, produced through the trapping of SEPs during geomagnetic activity. The elemental composition of ions in the outer radiation belt is similar to that of the solar wind up to energies of several tens of MeV. The ratio of Oxygen / Carbon is close to the value observed in the solar wind. This result does not favor an ionospheric source (Hovestadt et al. 1978). The inner belt is produced via the cosmic ray albedo neutron decay (CRAND) process (Singer 1958; Lazutin and Logachev 2009); a fraction of neutrons produced during the interaction of high energy cosmic rays with the Earth's atmosphere is backscattered and decay into protons and electrons (White 1973).

The inner and outer belts are separated by a slot region at $L=2.2-3.5$ during quiet periods, which shifts to lower $L$-values during disturbed periods (Kuznetsov et al. 2005). The peak flux of protons occurs near $L=1.3$, with lower energy fluxes peaking at higher $L$-shells. The proton belt $(L<1.7)$ is quite stable (Blanc et al. 1999). The protons are lost by Coulomb collisions with electrons in the plasmasphere, by charge exchange with hydrogen atoms, and by collision with atmospheric atoms. 
Secular changes in the geomagnetic field over hundreds of years may gradually increase the proton density by a factor of $\sim 10$ due to contracting drift shells (Selesnick et al. 2007). The proton flux displays a solar cycle variation which is in anticorrelation with the solar flux (Miyoshi et al. 2000). High solar activity causes heating and expansion of the upper atmosphere, leading to an increase in the collision rate and to proton losses. In the outer region $(L>3)$, variations in the proton flux have been observed during the interaction of an interplanetary shock with the Earth's magnetosphere. Shock related compression of the magnetosphere could accelerate protons of solar origin up to tens of $\mathrm{MeV}$ within tens of seconds (Blake et al. 1992; Hudson et al. 1995; Lorentzen et al. 2002; Looper et al. 2005).

The NASA models of radiation belts based on many satellite measurements of protons from 0.1 to $400 \mathrm{MeV}$ and electrons from 0.1 to $7 \mathrm{MeV}$ over a wide range of $L$-values are available on the website: http://modelweb.gsfc.nasa.gov/models/-trap.html.

The electrons in the outer belt are mostly of solar wind origin and they are accelerated inside the magnetosphere. Several possible mechanisms have been proposed ( $\mathrm{Li}$ and Temerin 2001; Friedel et al. 2002; Horne 2002). Betatron and Fermi acceleration as a result of transport across the magnetic field (radial diffusion towards the Earth) becomes much more efficient when ULF waves at frequencies of a few $\mathrm{Hz}$ are present (Elkington et al. 1999). This is corroborated by the high correlation between the occurrence of ULF waves, flux increases and the speed of solar wind (Mathie and Mann 2000; Horne et al. 2005). Recent observations show the peak particle density near $L=4.5$ and not in the outer region as required by inward radial transport (Iles et al. 2006; Chan et al. 2007; Shprits et al. 2007). This suggests the presence of a local acceleration mechanism (Horne 2007; Shprits et al. 2007).

The local acceleration can be achieved through the resonant wave-particle acceleration between energetic electrons and whistler mode waves (Horne and Thorne 1998; Horne et al. 2003a, b, 2005, 2006; Trakhtengerts and Rycroft 2008). Out of the various whistler mode waves present in the magnetosphere, chorus waves are preferred because (a) chorus waves are most intense between $L=4-6$ from midnight to the early afternoon MLT sector, corresponding to the peak in the flux density of the outer radiation belt (Meredith et al. 2001, 2003), (b) wave power increases with magnetic activity in a region where the ratio of the electron plasma frequency to the gyrofrequency is low, which is also a condition for the efficient diffusion of electrons to higher energies (Horne et al. 2003a), and (c) wave acceleration predicts an energy dependent "flat top" pitch angle distribution, which has also been observed during a magnetic storm (Horne et al. 2003b). During the resonant wave-particle interaction, the chorus waves grow at the cost of low energy $(\sim 10 \mathrm{keV})$ and small pitch angle electrons. At the same time waves also interact with much higher energy electrons at large pitch angles and accelerate them (Horne 2007; Shprits et al. 2006, 2007). Thus there is a transfer of energy from a large number of low energy electrons at small pitch angles to accelerate a fraction of the population at large pitch angle to higher energy.

Resonant wave-particle interactions also scatter particles into the loss cone (Trakhtengerts and Rycroft 2008), leading the loss of particles from the interaction region into the atmosphere. The waves involved can be plasmaspheric hiss (Abel and Thorne 1998; Meredith et al. 2006), electromagnetic ion cyclotron waves (Albert 2003; Summers and Thorne 2003; Glauert and Horne 2005), lightning generated whistlers and whistler mode waves from ground based radio transmitters (Abel and Thorne 1998).

The energetic electrons and protons gyrating along geomagnetic field lines penetrate deeper in to the atmosphere in the South Atlantic Anomaly region due to the weakness of the geomagnetic field there, and they pose a hazard to satellites in LEO (Low Earth Orbit) at that location. Precipitating energetic electrons stimulate the high frequency whistler 
mode emissions via the incoherent Cerenkov radiation process (Rothkaehl and Parrot 2005). Precipitating energetic electrons also generate hard X-rays through bremsstrahlung radiation (Feldman et al. 1996; Bucík et al. 2006). A LEO satellite detected enhanced (by about $28 \mathrm{~dB}$ ) high frequency emissions over the Euro-Asia region (Klos et al. 1997); these are thought to be caused by high energy $(0.5-1.5 \mathrm{MeV})$ precipitating charged particles from the radiation belt (Rothkaehl and Klos 2003). Precipitation of high energy particles may disturb the topside ionosphere and lead to enhanced turbulence in the ionospheric plasma (Rothkaehl and Parrot 2005).

Further, precipitating electrons cause localized ionization and changes of lower ionospheric plasma conductivity (Demirkol et al. 1999; Rodger et al. 2001). The feedback of magnetospheric Alfven waves and varying ionospheric conductivity leads to the creation of complex magnetosphere-ionosphere current systems (Pokhotelov et al. 2004). Kuznetsov et al. (2007) studied the distribution of both electrons and protons during geomagnetic storms between August 2001 and August 2003 by CORONAS-F measurements and showed that the charged particle fluxes decreased abruptly in the outer belt during the main phase of the storm and the slot region is filled. They found that a new belt is built up, and the lowest $L$-value to which the belt is shifted is given by $\sim\left|D_{\mathrm{st}}\right|^{1 / 4}$ (Tverskaya et al. 2003), where $D_{\text {st }}$ is the storm time ring current index. During the recovery phase the outer radiation belt recovers. In all the cases studied, the Earth's radiation belts exhibited variations for several days.

\subsubsection{Solar Wind}

The solar wind is tenuous magnetized plasma, a mixture of ions and electrons flowing away from the Sun. The solar wind and the IMF carried with it prove to be a key link between the solar atmosphere and the Earth's magnetosphere. On average, the solar wind at Earth orbit has a mean density of about $4 \mathrm{~cm}^{-3}$, a mean velocity $V_{\mathrm{SW}}$ of about $400 \mathrm{~km} / \mathrm{s}$, and a mean interplanetary magnetic field magnitude of $5 \mathrm{nT}$. The average direction of the interplanetary magnetic field along the Parker spiral in the ecliptic plane is at an angle of about $45^{\circ}$ from the radial direction (Hundhausen 1972; Hundhausen 1997) (see Fig. 2b).

Geomagnetic activity is primarily driven by magnetic reconnection between the IMF and the terrestrial magnetic field. The orientation of the IMF plays a dominant role for the geoeffectiveness of solar wind disturbances. As the dipole is close to being perpendicular to the ecliptic plane, it is primarily dependent on the southward component of the IMF, i.e. a negative value of $B_{Z}$, and the reconnection rate is proportional to the $y$-component of the motional electric field $\left(E=-V_{\mathrm{SW}} \times B_{\mathrm{IMF}}\right)$ of the solar wind (Vasyliunas 1975; Dungey 1981; Lakhina and Tsurutani 1998; Priest and Forbes 2000). Figure 7 shows a schematic of the solar-interplanetary-magnetosphere coupling during solar maximum years. Different field components and their flow directions are shown (Singh 2003). During reconnection, a significant transfer of energy, momentum and mass from the solar wind to the Earth's magnetosphere takes place. Coherent solar wind structures containing a southward magnetic field and high velocities are thus the most efficient drivers of dayside magnetopause reconnection and hence of space weather events.

High speed solar wind streams encountering the Earth most often originate from low latitude coronal holes (mostly during the quiet Sun) having funnel-shape magnetic structures, expanding in the corona with a narrow neck in the photosphere (Gabriel 1974). The solar wind plasma is considered to be supplied by plasma emanating from the many small magnetic loops forming and crowding the funnel and extending to a few $1,000 \mathrm{~km}$ in height above the photosphere. Through magnetic reconnection, plasma is fed from all sides 


\section{EARTH'S MAGNETOSPHERE}

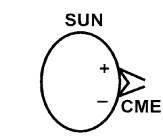

CME: CORONAL

MASS

EJECTION
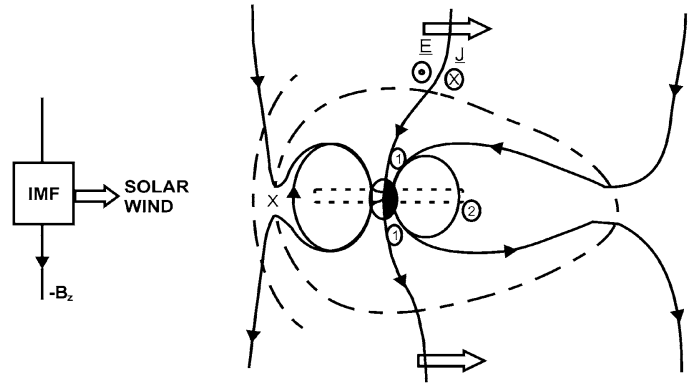

IMF: INTERPLANETARY MAGNETIC FIELD

$-B_{z}$ : SOUTHWARD COMPONENT OF IMF

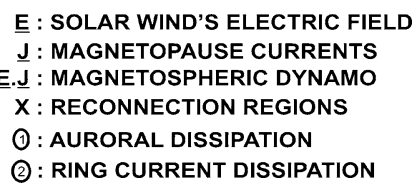

E : SOLAR WIND'S ELECTRIC FIELD

J : MAGNETOPAUSE CURRENTS

(1) : AURORAL DISSIPATION

(2): RING CURRENT DISSIPATION

Fig. 7 Schematic of the solar-interplanetary-magnetosphere coupling during solar maximum years, during which a coronal mass ejection is the most important source for interplanetary and magnetospheric disturbances (credit: Singh 2003)

to the funnel, where it is accelerated and finally forms the fast solar wind ( Tu et al. 2005; Balogh et al. 2008).

The slow solar wind originates from above the more active regions of the Sun. During declining and minimum phases of the solar cycle, the slow solar wind emanates from the solar equatorial region, whereas the fast solar wind comes from the polar regions (McComas et al. 2003). High speed solar wind is often associated with strong Alfvenic fluctuations, leading to strong fluctuation of IMF $B_{z}$ and the solar wind velocity. These waves are effective drivers of medium-level magnetic activity in the high latitude magnetosphere and in the ring current (Tsurutani and Gonzalez 1987). Sometimes density enhancements and magnetic field compression coincide with the start of the increase of wind speed (Tsurutani et al. 2006). During the declining phase of a solar cycle, the coronal holes extend to low latitudes, sometimes even reaching the ecliptic plane.

The CMEs traveling through the interplanetary medium are transient disturbances which happen at random intervals. These disturbances are superimposed on the background solar wind. This is the persistent flow of ionised solar plasma and a remnant of the solar magnetic field that spreads out in the Parker (1957) spiral pattern. Although it is always present, the solar wind does not always flow at the same speed; different parts travel faster than others. As a result the faster parts catch up with the preceding slower parts, so that alternating regions of compressed plasma and fields, and less dense regions, are formed. The passage of these regions called corotating interaction regions (CIRs) as they sweep by the Earth's magnetosphere induces geomagnetic activity through variations of the solar wind pressure and magnetic field orientation at the Earth's magnetopause (Singh 2003).

When the high speed solar wind emanating from a coronal hole runs into the slower solar wind, there is a compression of the plasma and the magnetic field; this forms a Corotating Interaction Region (CIR) (Crooker et al. 1999). A schematic diagram showing the structure of a typical high speed stream with the embedded CIR is shown in Fig. 8 
Fig. 8 Schematic showing the structure of a typical high-speed stream including the embedded corotating interaction region (CIR) (credit: Borovsky and Denton 2009)

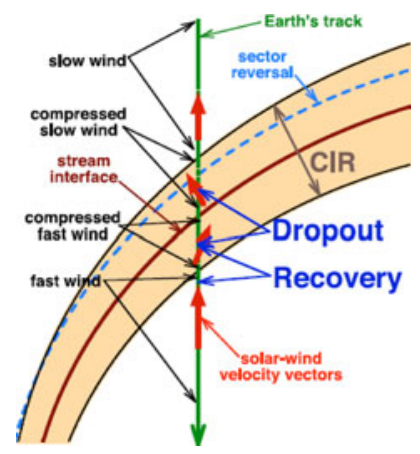

(Borovsky and Denton 2009). The CIRs seldom have fast shocks or continuous, strongly southward interplanetary magnetic field (IMF) and thus drive only moderate geomagnetic activity (Scholer 1999; Alves et al. 2006; Borovsky and Denton 2006). It is generally believed that geomagnetic disturbances recurring at 27 day intervals could be associated with CIRs.

Studies show that both CMEs and CIRs produce magnetic fields significantly larger than the normal IMF (Lindsay et al. 1995). CIRs associated with compressed plasma and fields often produce larger dynamic pressure than the CMEs (Watari 1997). The increase of IMF fluctuations in CIRs is due to the presence of large amplitude Alfven waves within the body of the corotating streams (Tsurutani et al. 1995a), which has been confirmed by Ulysses' observations (Tsurutani et al. 1995b). Ulysses observations showed the tilted latitudinal structure of the CIRs; the tilt of the CIRs decreases with increasing distance from the Sun (Gosling et al. 1993). Thus, high speed solar wind emanating from out of the ecliptic plane, particularly in the vicinity of magnetic sector boundaries (where a CIR is easily formed) can also become effective in producing geomagnetic disturbances (Gosling et al. 1976; Scholer 1999).

\subsection{Interplanetary Space}

The sudden ejection of plasma and magnetic field structures from the Sun's atmosphere in the form of CMEs influences the Earth's environment to produce space weather hazards. In addition, galactic cosmic rays, originating from far beyond the solar system are scattered by the IMF and are at maximum at solar minimum and at minimum at solar maximum.

Beyond the protective shield of the Earth's atmosphere and magnetosphere, there are sources of radiation that can be a serious hazard to humans and electronic equipment. High energy charged particles in space include the sudden intense bursts of the solar energetic particles (SEPs) that can last several days. Studies show that pre-existing energetic particles, preceding CMEs and the density structure of the extended corona all have an influence on the production of SEP (Kahler and Vourlidas 2005). The many processes involved in the development of SEP events include the acceleration and transport of particles in a time dependent system formed by the propagating CME-driven shock, the associated evolving magnetic field topology and the generation of magnetic field fluctuations (Webb et al. 2001).

Major features of shock-associated SEP events are reproduced by detailed models of the evolving shock properties, wave-particle interactions and particle transport processes (Lee 2005; Vainio and Laitinen 2007, 2008; Ng and Reames 2008; Vainio et al. 2009). 
The distribution of particles streaming along magnetic field lines produces resonant Alfven waves (Melrose 1982). At high particle intensities, the resonant wave intensity becomes sufficiently large to scatter the particles that come behind and reduce their streaming. This process traps the particles near their source and also puts a limit on the outward flow of the streaming particles. These trapped particles around a shock are accelerated and the appearance of their intensity-time profile will depend on the solar longitude of the observer relative to the CME (Kahler 2001). This effect arises because of the spiral nature of the magnetic field. An observer's magnetic connection to the shock swings eastward with time, either approaching or receding from the intense nose of the shock (Reames 2001). The shock strength may also decrease with time as the shock expands radially. The flow of particles from the distant shock is limited in intensity due to scattering of self generated Alfven waves; very large intensity peaks can occur when a strong shock from the central meridian of the Sun passes over the spacecraft. Events with high intensities of $>100 \mathrm{MeV}$ protons at shock peaks are rare, may be once in a decade. But their effect is severe on a long duration space mission. Further, the rarity of such events makes it difficult to study them and to forecast the probability of their occurrence.

The fast CMEs often drive large-scale density waves out into space which eventually steepen to form collisionless shock waves, similar to the bow shock in front of the Earth's magnetosphere. The shock front is the outer boundary of the plasma sheath that results from the compression, deflection and heating of the ambient solar wind by the ensuing ejecta (Tsurutani et al. 1988). The sheath may contain substantial distortion of the interplanetary magnetic field due to field line draping (McComas et al. 1988) around the ejecta cloud pressing from behind.

For about one third of all shocks driven by interplanetary CMEs (ICMEs), the succeeding plasma exhibits to an in situ observer the topology of magnetic clouds (MCs), such as magnetic bottles, tongues or bubbles. MCs are localized regions in interplanetary space having enhanced magnetic field strength, lower proton temperature (as compared to the background solar wind) and a large scale large-angle smooth rotation of the magnetic field vector (Burlaga 1991). Analyzing data from different interplanetary spacecraft, Burlaga et al. (1981) proposed a locally cylindrical topology with circular magnetic field lines around a common cloud axis. Figure 9 shows a sketch of the MC shape as a cylindrical flux-rope (Watermann et al. 2009b). The simple flux-rope topology of MCs makes them useful for MHD modeling (Osherovich and Burlaga 1997). As the density inside the MCs

(a)

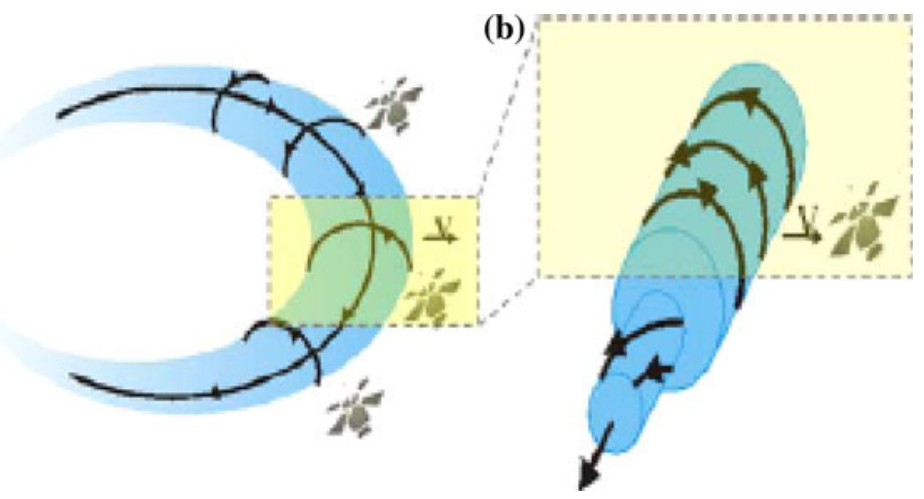

Fig. 9 Topology of magnetic clouds as cylindrical flux ropes, a global view b local view (credit: Watermann et al. 2009b) 
is often extremely low, the force-free configuration proposed by Goldstein (1983) and developed by Feng et al. (2006) could be more useful in identifying the configuration and boundaries of MCs. Non-force free models have also been developed. All of these considered a cylindrical cloud with circular (Cid et al. 2002) or elliptical (Mulligan and Russell 2001) cross sections. Vandas et al. (2006) proposed an elliptical cross section model with a force-free configuration. To date, there is no single model which can produce all the observed features of MCs.

CMEs originating from the solar disk and propagating directly along the Sun-Earth line appear as halo CMEs (Howard et al. 1982, 1985). Full halo CMEs have an apparent angular width of $360^{\circ}$, and, if coming from the front-side of the Sun, give an early warning of possible geomagnetic disturbance. Associated activities on the solar disk in the form of post-eruptive loops, disappearing filaments (erupting prominences) or flare signatures have been observed (Tripathi et al. 2004). The ICME corresponding to a front-side halo CME detected in the field view of SOHO/ LASCO placed on the Sun-Earth line may take a few days to reach the magnetosphere of the Earth (Brückner et al. 1995). However, not every halo CME will reach the Earth. Schwenn et al. (2005) studied 181 front-side halo CMEs and showed that $15 \%$ did not show any in situ discernable signature. The longitudinal location of the source is of vital importance. Events occurring close to the limb and on the back side of the Sun have little probability of arrival at the Earth, whereas those originating close to the Sun-Earth line have a high probability. Even if halo CMEs originating near the limb reach the magnetosphere, then they tend to be less geoeffective and only a shock may be observed (Manoharan et al. 2004), because the angular extent of the shock is larger than that of the corresponding CME (Akasofu and Fry 1986).

Rodriguez et al. (2009) analyzed three front-side full halo CMEs that occurred on April 4, 2000, June 6, 2000 and September 12, 2000 (close to the maximum solar activity period) and showed their speeds to be higher than $1,100 \mathrm{~km} \mathrm{~s}^{-1}$. Out of the three events, one originated close to the limb and produced a severe geomagnetic storm, whereas the other two started off close to the central meridian but produced weak and moderate geomagnetic storms. This non-typical geomagnetic response has been explained in terms of the nonradial direction of the eruption derived from the mass distribution in the halo (Moon et al. 2005), the passage of the Earth through a leg of an interplanetary flux-rope, and strong compression at the eastern flank of a propagating interplanetary CME during its interaction with the ambient solar wind (Rodriguez et al. 2009).

\subsection{Magnetosphere}

A magnetosphere (see Fig. 10 which illustrates the Earth's magnetosphere) is the cavity formed by the interaction of the solar wind and the interplanetary magnetic field with the intrinsic magnetic field or ionized upper atmosphere of a planetary body. In the terrestrial case, the interaction is dominated by the strong intrinsic quasi-dipolar geomagnetic field. While the magnetospheric field in general excludes the solar wind plasma from the magnetosphere, at times the solar wind can gain entry into the magnetosphere through the magnetopause. This occurs when the IMF and the magnetospheric fields are antiparallel and magnetic "reconnection" creates an "open magnetosphere" configuration (Dungey 1961). Plasma entry is strongly modulated by the orientation of the interplanetary magnetic field, and is enhanced during periods of southward IMF (Russell and McPherron 1973). While the plasma enters mostly through the dayside magnetopause, some of the particles gain entry through the nightside magnetopause boundary layers at low latitudes (Paschmann 1997). During northward IMF, high latitude reconnection over the magnetic 


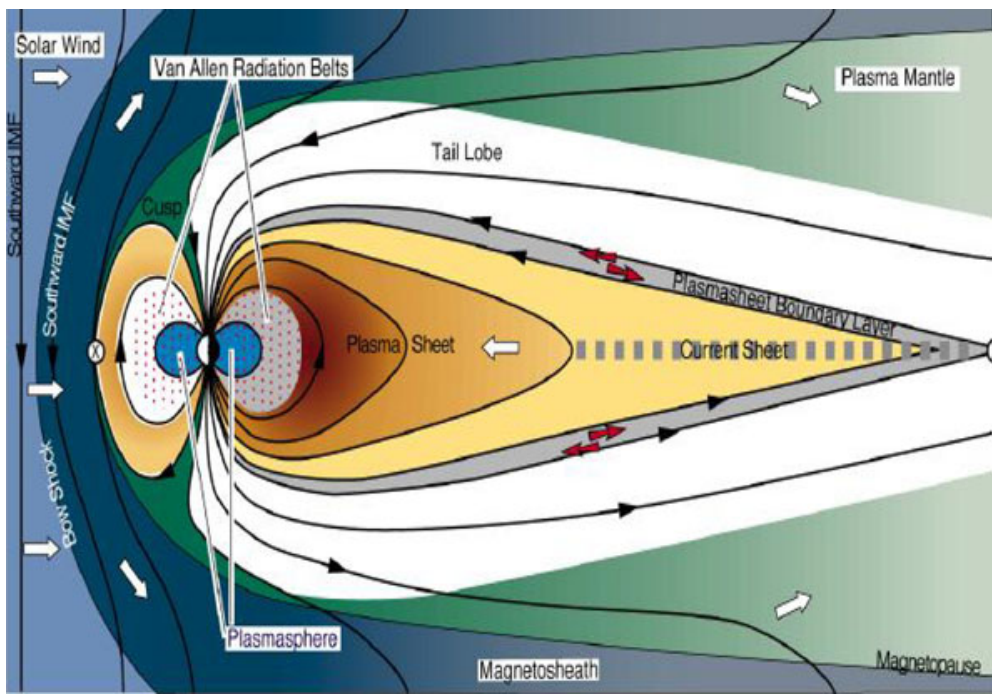

Fig. 10 Schematic diagram of the magnetosphere (http://space.rice.edu)

pole allows for particle entry at the high-latitude plasma mantle. A minor portion of the energy, estimated to be about $10 \%$, enters via viscous interaction processes (Axford and Hines 1961).

The solar wind plasma, consisting mostly of protons and a small percentage of doubly charged helium and other heavier ions, populates much of the outer magnetosphere through these processes. The IMF and solar wind pressure also control both the cusp position and its extent in the dawn-dusk and North-South directions (Dubinin et al. 2002). The cusps are believed to be another place of transport of plasma into the magnetosphere. Analyzing the POLAR spacecraft data, Chen et al. (1998) presented a Cusp Energetic Particle (CEP) event having (a) a decrease in magnetic field magnitude in the dayside cusp, (b) a more than one order of magnitude increase in intensity of 1-10 keV ions, and (c) a substantial (more than $3 \sigma, \sigma$ being the standard deviation of the measured data) increase in intensity above background for $>40 \mathrm{keV}$ ions (dominated by protons). These CEP events have shed light on the origin of the energetic particles in the magnetosphere (Fritz et al. 2003 a, b) and in upstream ion events (Chen and Fritz 2002a, b).

The origin of energetic particles in the CEP events was debated to be (i) locally energized in the high-altitude cusp (Sheldon et al. 1998; Chen et al. 1998), (ii) energized particles from the ring current source in the geomagnetic tail associated with substorms and drifting into the cusp (Blake 1999; Antonova et al. 2000; Chen and Fritz 2001), or (iii) particles energized at the quasi-parallel bow shock and subsequently transported into the cusp (Chang et al. 2000). The solar wind plasma in the high altitude dayside cusp regions causes extremely large diamagnetic cavities, which are identified by a combination of low magnetic field strength and high solar wind plasma intensity (Chen et al. 1998). These cavities are associated with particles of energies from $20 \mathrm{keV}$ up to $10 \mathrm{MeV}$. Turbulent electric fields $\sim 10 \mathrm{mV} / \mathrm{m}$ were also observed in the cusp (Chen and Fritz 1999). Observational facts reveal that cusps are regions of turbulent magnetic fields filled with shocked solar wind plasma and, occasionally, with plasma of ionospheric origin (Lemaire and Gringauz 1998; Chen and Fritz 1999). 
Fu et al. (2005) analyzed RAPID instrument data collected by Cluster spacecraft; they showed a continuous sunward flow which could be a result of high latitude reconnection when the IMF was northward. The high speed of flow might be a consequence of acceleration of the plasma in the presence of a strong magnetic field during the reconnection process (Dungey 1961). This sunward flow is termed reverse convection (Crooker et al. 1992). High latitude reconnection can also open up a path for charged particles to enter or escape from the magnetosphere (Zong et al. 2002). Lavraud et al. (2005) have presented an overview of Cluster observations in the high latitude cusp region, showing that it is filled with magnetosheath plasma of solar wind origin. Both precipitating and mirroring components were observed. However, the dayside plasma sheet was mainly populated with ions of magnetospheric origin. Usually, the plasma flow during northward IMF is such that the plasma mantle may not form (Sckopke et al. 1976). Observations (Lavraud et al. 2005) support to this hypothesis. The presence or absence of the plasma mantle region strongly depends on the nature of the IMF and the subsequent large scale plasma convection in the cusp regions.

The above discussion and observations suggest that convection in the cusp is strongly linked with the interplanetary conditions. Therefore, in order to understand exactly how solar wind plasma enters the magnetosphere, one has to know precisely the global structure, the magnetic field topology and dynamics of the high-latitude cusp region and its surroundings. It is suggested that numerical simulations including input from Cluster experiments should be performed to understand the dynamics of the cusp regions.

In addition to the solar wind, the Earth's upper atmosphere contributes both to the neutral and charged particle density in the magnetosphere. The neutral exosphere, or the upward extension of the atmosphere, fills the near-Earth space with gas of an exponentially decreasing density (Tinsley et al. 1986). While the neutral atoms and molecules are not influenced by the electromagnetic forces, the geocorona (envelope of ionized gases, especially hydrogen, surrounding the Earth at the outer limit of the atmosphere) is significant to space physics processes in at least two ways-Coulomb collisions and chargeexchange processes with neutral atoms are significant loss mechanisms for energetic charged particles in the inner magnetosphere (Fok et al. 1995), which contribute to the recovery to quiet conditions after a magnetic storm. Further, the slow neutral atoms charge exchanging with fast charged particles create a population of high-speed neutrals and low speed ions.

During active times, when the flux of energetic charged particles is sufficiently high, this mechanism provides a means of imaging the inner parts of the magnetosphere (Bertaux et al. 1989; Williams et al. 1992). The extreme ultraviolet (EUV) imager (Sandel et al. 2001) onboard the Imager for Magnetopause-to-Aurora Global Exploration (IMAGE) satellite produces full global images of the Earth's plasmasphere by remote-sensing solar $30.4 \mathrm{~nm}$ light wave resonantly scattered by $\mathrm{He}^{+}$ions in the plasmasphere. Plasmaspheric images are obtained every 10 min with a spatial resolution of $0.1 R_{E}$ (Goldstein 2006). Hence plasmaspheric dynamics can be followed from storm onset and erosion through recovery and refilling. Such results have complemented more than 40 years of in situ and ground observations (Darrouzet et al. 2009, Singh et al. 2010).

\subsubsection{Magnetospheric Dynamics}

Magnetospheric dynamics play a major role in space weather studies (Pulkkinen 2007). Solar wind mass, momentum and energy enter the magnetosphere via magnetic reconnection at the magnetospheric boundaries in regions where the interplanetary and terrestrial 
fields are antiparallel (Dungey 1961), by via viscous interactions along the boundary (Axford and Hines 1961), and via the magnetospheric cusps (Fritz and Fung 2005). In a quiescent situation, the energy inflow is gradual, the energy is dissipated in the ionosphere, and magnetic flux opened at the dayside reconnection process is closed by a quasi-static reconnection process in the distant magnetotail at up to about 100-200 $\mathrm{R}_{\mathrm{E}}$ from the Earth.

Energy transfer is most efficient when the reconnection process takes place at the dayside magnetopause, which occurs during periods when the interplanetary field points southward and is thus antiparallel to the intrinsic geomagnetic field (Akasofu 1981; Laitinen et al. 2006). Variability in the North-South component of the interplanetary magnetic field causes episodic energy loading dissipation cycles, termed magnetospheric substorms (Baker et al. 1996; Sergeev et al. 1996a). Magnetospheric substorms and geomagnetic storms are the most commonly occurring response to enhanced solar wind driving, but the magnetosphere can have other dynamic states. Steady convection intervals are periods of steadily southward IMF and steady, relatively slow solar wind flow that drives continuous, low level auroral activity without any evident substorm expansion phase (Sergeev et al. 1996b). Stronger levels of driving may lead to saw tooth oscillations, which are large scale substorms associated with strong, longitudinally extended, quasi-simultaneous injections at geostationary orbit every 2-3 h (Henderson et al. 2006; Pulkkinen et al. 2006).

Substorms typically occur at a rate of four to five per day, each lasting typically $2 \mathrm{~h}$ (Akasofu 1968; Kintner et al. 2008; Daglis 2001). They are initiated by enhanced dayside reconnection and hence increased energy input to the magnetosphere. This causes a configuration change in the magnetosphere including the enhancement of the magnetospheric current systems and the formation of a thin and intense current sheet from near-geostationary distance outward. It can be centered at local times far from midnight, can apparently be localized to one meridian, and can have multiple onsets centered at different local times (McPherron 1970; Pulkkinen et al. 1992). The current sheet at the centre of the magnetotail has a thickness of only a few $100 \mathrm{~km}$; the magnetic field component normal to the current sheet becomes very small, only a few nT (Sergeev et al. 1993). As this thin current sheet grows unstable, a rapid dissipation process quickly expands to a large scale reconnection event whose effects are observable over a large portion of the coupled magnetosphere-ionosphere system (Hones 1979). Because the reconnection region is limited in the cross-tail direction, the flow shear between the fast outflow from the reconnection region and the ambient eastward plasma flow within the plasmasheet creates a pair of field-aligned current sheets, where the current flows to the ionosphere at the eastern edge of the reconnection region and out from the ionosphere at the western edge (McPherron et al. 1973). On the ground at high latitudes, magnetospheric substorms create disturbances in the geomagnetic field caused by the field-aligned current systems and auroral electrojet currents in the ionosphere where they close (Baumjohann et al. 1981).

Geomagnetic storms are largest magnetospheric disturbances which are associated with many space weather phenomena. While substorms can occur without magnetic storms, almost all storms include substorm activity. The storm occurrence is normally indicated by a large decrease of the $D_{\text {st }}$ index which measures the variations in the geomagnetic NorthSouth component at equatorial stations. It is constructed using the horizontal component of the geomagnetic field observed at low to mid latitudes (Surgiara 1965; Sugiara and Kamei 1991). It was introduced as a measurement of the intensity of the ring current (Surgiara 1965). $D_{\text {st }}$ is always negative during storms. A larger negative $D_{\text {st }}$ means a more intense storm and generally a more intense ring current. However, $D_{\text {st }}$ is not a perfect measure of ring current intensity because it is influenced by other magnetospheric currents, such as the 
cross-tail current (Daglis et al. 1999). Intense geomagnetic storm events, typically characterized by a peak $D_{\text {st }}<-100 \mathrm{nT}$ (Gonzalez et al. 1994), have the potential to be the most disastrous to the near-Earth space environment.

As mentioned earlier, the major interplanetary drivers of intense magnetic storms are CMEs (Echer et al. 2006; Gonzalez et al. 2007). Corotating interaction regions (CIRs) also lead to storm development. However, the CIR-driven storms exhibit a weaker intensity than the CME-driven ones; they mainly develop during the declining phase of the solar cycle (Tsurutani et al. 2006; Gonzalez et al. 2007). The interaction of the inner magnetosphere storm-associated processes with the magnetotail substorm-associated processes are highly complex, and presently under active study. Whether the substorm-associated magnetic field variations and their associated electric fields are necessary for the buildup of the ring current energy density is debatable (McPherron 1997; Daglis and Kamide 2003). Some arguments indicate that convection alone can account for the ring current increase (Ebihara and Ejiri 2003), whereas others have concluded that an energization process leading to charged particles with energies exceeding $100 \mathrm{keV}$ needs smaller-scale, time varying electric fields (Ganushkina et al. 2005). The theory of wave-particle interactions in the magnetosphere has been reviewed recently by Trakhtengerts and Rycroft (2008). A probabilistic assessment on likely future storm occurrence is one of the top priorities for space weather forecasting (Tsubouchi and Omura 2007).

\subsection{Magnetosphere-Ionosphere Dynamics}

The terrestrial ionosphere produced and maintained by solar extreme ultraviolet (EUV) radiation and X-radiation extends from $70 \mathrm{~km}$ altitude to several thousand kilometers (Rees 1989; Kivelson and Russell 1995; Prolss 2004; Kintner et al. 2008; Schunk and Nagy 2009). The ionosphere poses an interesting challenge for many radio systems that make use of it for signal transmission. Being a magneto-ionic medium imbedded in a background neutral atmosphere, it exhibits very interesting properties, including refraction, anisotropy, dispersion and dissipation. An understanding of ionospheric properties (Bailey et al. 2000; Denton et al. 2009) provides information about a wide range of solar-terrestrial interactions, and has significant space weather implications.

Once the interplanetary energy has coupled to the magnetosphere, magnetospheric plasma convection is enhanced and geomagnetic field-aligned currents accelerate plasma from the magnetotail into the polar ionosphere. The precipitation of charged particles into the polar atmosphere excites visual auroras, and increases the conductivity of the lower ionosphere ( $\mathrm{D}$ and $\mathrm{E}$ regions) in the auroral electrojet. Magnetospheric energy introduced into the polar region heats the thermosphere, changing the distribution of atmospheric constituents and hence the ionospheric electron density profile (Denton et al. 2009). This generally appears as reduced ionospheric critical frequencies (i.e. reduced plasma frequencies, for the ordinary mode of propagation) and increased heights of maximum electron density (Ondoh and Marubashi 2001; Hunsucker and Hargreaves 2003; Denton et al. 2009).

Geomagnetic storms represent the single most important space weather phenomenon and also are an exciting and rewarding topic that covers an interesting spectrum of ionosphere-magnetosphere interactions. Until the early 1970s, there was no evidence of the now well known fact that the storm time ring current is replete with oxygen ions of ionospheric origin (Shelley et al. 1972; Balsiger et al. 1980; Daglis et al. 1999), whereas previously they were thought to be of solar origin. Based on the experimental and analytical evidence of $\mathrm{O}^{+}$injection into the ring current, Daglis (1997a) concluded that the 
larger the storm is (as quantified by the $D_{\mathrm{st}}$ index) the larger the $\mathrm{O}^{+}$contribution to the ring current; the $D_{\text {st }}$ magnitude and the $\mathrm{O}^{+}$contribution to the ring current increase at same time. Daglis (1997b) suggested that the role of $\mathrm{O}^{+}$(the major outflowing ionospheric ion) in the evolution of intense storms is to cause the rapid final enhancement of the ring current at storm maximum and to induce an equally rapid initial decay of the ring current. This may be the cause of the two steps observed in the development (Kamide et al. 1998) and recovery (Noël 1997) of intense storms.

The enhanced ring current and varying auroral currents cause large variations in the time rate of change of the geomagnetic field, which in turn induces electric potential differences across large areas of the Earth's surface (considering it to be a conductor) and across cables (used for communication systems, or systems that are grounded to the Earth such as power grids, etc). The enhanced earth potential may lead to overruns of the compensating voltage swings that are designed in power supply systems (Anderson et al. 1974) that are being used to power the signal repeaters and regenerators used in optical fibre transmissions. Ring current enhancements can directly produce potential drops depending upon the nature and the orientation of underground Earth conductivity structures.

The decrease in $D_{\mathrm{st}}$ index is linearly related to the total particle energy injected from the ionosphere into the inner magnetosphere driving the ring current and ionization changes. Thus magnetic activity indices may also be considered as drivers of the ionospheric perturbations expressed by a positive phase (increasing electron and ion population densities) followed by a negative phase (depletion of ionization) (Gulyaeva and Stanislawaka 2010). Thus geomagnetic storms are related to the perturbations in ionospheric ionization. However, not all the perturbations in the ionospheric ionization are associated with geomagnetic storms, because variations in solar UV and EUV energy flux will also cause variations in ionospheric ionization.

\section{Space Weather Effects}

CMEs and ICMEs are the main causes of severe space weather at Earth which damages technological infrastructures in space as well as on the ground. Space and terrestrial technologies are increasingly sensitive to solar storms and the resulting changes to the Earth's radiation environment in the magnetosphere (Lilensten 2007; Bothmer and Daglis 2007). Here, we summarize some major effects of space weather. Table 2 (Koskinen et al. 2001) lists some space weather effects and their relations to the space environment.

\subsection{Ionospheric Effects}

The ionosphere significantly affects the propagation of radio frequency (RF) signals which pass through it or are reflected by it (Cannon 1994a, b). The effects are many and varied, but include absorption, refraction, retardation and scintillation. At frequencies above $\sim 1 \mathrm{MHz}$, the lower $\mathrm{D}$ region causes absorption and the higher $\mathrm{E}$ and $\mathrm{F}$ regions cause other effects. The diverse set of affected systems includes ground-ground high frequency (HF) communications, ground-space radio communications (e.g., for telemetry or altimetry), global positioning system (GPS)—particularly single or multiple frequency navigation systems, and space based radars (Goodman and Aarons 1990). HF communications and radar systems rely on the ionosphere for their operation but also have to contend with its effects. The performance of most systems is degraded by ionospheric variations, but for 
Table 2 Lists some space weather effects and their relations to the space environment (credit: Koskinen et al. 2001)

\begin{tabular}{lll}
\hline Space weather effect & Space weather parameters & Material/object parameters \\
\hline Drag & Total density & Cross-sectional frontal area \\
& Fluid velocity & $\begin{array}{l}\text { Mass } \\
\text { Relative velocity }\end{array}$ \\
& Shape \\
Atom displacement & Particle flux & Shielding, material, geometry \\
Dark current increase & Particle flux & Shielding \\
& Photon flux $(\mathrm{UV}, \mathrm{X}, \gamma)$ & Absorption \\
Surface charging & Electron flux $(<300 \mathrm{keV})$ & Secondary emission \\
& Proton flux $(<8 \mathrm{MeV}) \mathrm{UV}$ flux & $\begin{array}{l}\text { Photoelectric emission } \\
\text { Geometry }\end{array}$ \\
Internal charging & Electron flux $(>300 \mathrm{keV})$ & Shielding, material, geometry \\
SEEs & Ion flux $(>100 \mathrm{MeV})$ & Technology (CMOS) \\
& Proton flux $(>10 \mathrm{MeV})$ & Cell nature (eyes, gonads, bone marrow) \\
Human effects & Ion flux $(>100 \mathrm{MeV})$ & Cross-sectional area, optical \\
Orbital elements & Proton flux $(>10 \mathrm{MeV})$ & properties, shape \\
& Atmospheric scale height & Relative velocity, size, type of surface \\
Electrostatic discharges & Meteoroids and debris & Technology, type of materials \\
& UV radiation, plasma &
\end{tabular}

certain specialist systems detailed ionospheric knowledge can be of great benefit (Schunk and Sojka 1996; Bilitza 2001; Song et al. 2001; Scherer et al. 2005).

\subsubsection{Effects on Communications}

A century after Marconi's feat of trans-Atlantic radio communication, the ionosphere remains both a facilitator and a disturber in numerous communication applications. The military, as well as police and emergency agencies in many nations, continue to rely on wireless links that make extensive use of radio frequencies from several $\mathrm{kHz}$ to hundreds of $\mathrm{MHz}$ that use the ionosphere as a reflector. At higher frequencies ( $\sim$ few GHz, typical operating frequencies for mobile telephone systems) the production of bubbles in the ionosphere, prevalent in equatorial and auroral regions of the Earth, are a prime source of scintillations in satellite-to-ground signals. Distributed ionospheric currents during geomagnetic active periods can also be the cause of considerable problems, at all geomagnetic latitudes, for navigation or communication systems.

The basic physical chain of events behind the production of large potential differences across the Earth's surface begins with greatly increased and time varying electrical currents flowing in the magnetosphere and ionosphere. The temporal and spatial variations of these increased currents then cause large variations in the time rate of change of the magnetic field at Earth's surface. The time variations in the field in turn induce potential differences across large areas of the surface that are spanned by cable communication systems, so inducing currents in them (Pirjola 1998; Boteler et al. 1998; Pirjola 2007). Telecommunications cable systems use the Earth itself as a ground return for their circuits, and these 
cables thus provide highly conducting paths for concentrating the electrical currents that flow between these newly established, but temporary, Earth batteries (Lanzerotti 2001).

The precise effects of these anomalous electrical currents depend upon the technical system to which the long conductors are connected. In case of long telecommunication lines, the Earth potentials can cause overruns of the compensating voltage swings that are designed into the power supplies (Anderson et al. 1974). At present, the time variations and spatial dependencies of these currents are not well understood or predictable from one geomagnetic storm to the next. This is of considerable importance since the induced Earth potentials are very much dependent upon the conductivity structure of the Earth underlying the affected ionospheric regions. This area of research involves a close interplay between space plasma geophysics and solid Earth geophysics.

\subsubsection{Effects on Navigation Systems}

Navigation systems are adversely affected by space weather. Aeroplanes and ships use very low frequency (VLF) radio signals from transmitters to determine their positions. During solar events and geomagnetic storms, the system may give navigators information that is inaccurate by as much as several km (Filjar 2008; www.swpc.noaa.gov/primer). If navigators are alerted that a geomagnetic storm is in progress they can switch to other systems. Global positioning system (GPS) devices are affected by the sudden variations in ionospheric density caused by space weather because a radio receiver's position is computed using code and carrier phase information contained in the satellites' RF signals. Since 1993, 24 or more GPS satellites have provided worldwide coverage and positioning capabilities (Skone and Yousuf 2007).

\subsection{Effects on Satellites}

Space weather has severe impacts on satellites (Tribble 1995; Hastings and Garrett 1996), rocket launchers (Boscher et al. 1998; Bourdarie and Bourrieau 1999) and GPS/navigation systems (Goodman and Aarons 1990; Wellenhof et al. 2001). Radiation effects depend on the energy of the radiation, from galactic cosmic rays, solar energetic particles and radiation trapped in the Earth's Van Allen belts (Bourrieau et al. 1996; Crosby et al. 2006). A number of spacecraft anomalies and failures following significantly enhanced fluxes of high energy radiation during interplanetary disturbances have been identified (Baker et al. 1996, Reeves et al. 1998; Barbieri and Mahmot 2004 and references therein). Dorman et al. (2005) found a clear difference between satellite anomaly probability and various physical characteristics of the interplanetary space, geomagnetic field and energetic particles of different energy and type.

The magnetic disturbances associated with space weather events may produce changes in the local magnetic field, leading to altitude determination problems with satellites and spacecraft that use magnetic guidance. The effect can be particularly large, e.g., when a geostationary satellite may enter the solar wind when extreme solar wind pressure compresses the dayside magnetopause severely.

The low energy (few eV and above) components interacting with the surfaces of the satellite can produce surface charging on the material used to encase the satellite (Garrett 1981). The plasma flux can be highly variable in time and intensity, which may result in different charging levels. In the absence of good electrical connection between the various surface materials, differential charging on the surface can produce very high voltages 
( $\sim 800 \mathrm{~V}$ ) leading to breakdown discharges (Lanzerotti 2007). These discharges may damage components and subsystems of the spacecraft (Koons 1980; Vampola 1987).

The cross-magnetosphere electric field during enhanced geomagnetic activity convects the plasma sheet towards the Earth, to within the geosynchronous orbit. In such a case surface charging effects may occur, especially in the local midnight to dawn sector of the orbit (Mizera 1983).

High energy $(>\mathrm{MeV})$ charged particles can penetrate through a satellite skin deep into its interior. The intensities of these particles may change by many orders of magnitude over the course of minutes. The penetrating charged particles produce transient electrical pulses leading to upsets and latchups in the signal and control electronics of satellites (Crosby et al. 2006). Such particles may also cause the build up of electrical charges on insulating (dielectric) materials such as those used in coaxial cables. Such a charge build up may ultimately result in electrical breakdown and cause not only electromagnetic interference but also damage to the electronic components (Lanzerotti 2007).

Low Earth orbit (LEO) satellites are strongly affected by the atmospheric density which determines the drag acting on the satellite and hence the lifetime of the satellite. The upper atmospheric density responds to the solar activity, depending on season, local time, latitude and magnetic storm conditions. During periods of high solar activity, the upper atmosphere expands. If magnetic activity is also triggered then intense electric currents produce additional heating in these regions and cause drag on LEO satellites (Flury and Contant 2001; Klinkrad 2007).

The impact of light meteoroids and space debris left in orbit from space launches and from satellites that may breakup can disorient or even may cause a total loss of a satellite (Daglis 2001; Carlowicz and Lopez 2002). The US Air Force systematically tracks a large number of pieces of space debris that are circling the Earth, most of which are in LEO (Lanzerotti 2007). Such hazards are not usually classified as space weather hazards.

Table 3 (Koskinen et al. 2001) categorizes some components of space environment and their effects on spacecraft.

\subsection{Space Radiation Effects}

With the increasing use of microelectronics of ever diminishing size, space systems are becoming increasingly susceptible to space weather events due to the highly ionizing interactions of cosmic rays and solar energetic particles. Humans in space are normally well protected by the effective shielding in space stations. However, flashes in the astronaut's eyes due to high energy charged particles have been seen when they are inside the Space Shuttle (Allen and Wilkinson 1993). During extra vehicular activities (EVAs), effects similar to those experienced inside space vehicles are possible, but with the shielding being much less (Lemaignen 1988). Charging effects can also appear when astronauts cross the boundary between sunlight and shadow (Koskinen et al. 2001). The charged particle radiation (over the entire range of energies) that permeates the near-Earth space environment remains a difficult problem for the design and operations of these and other space based systems (Shea and Smart 1998; Koons et al. 1999).

The fluxes of low energy (few eV to few keV) charged particles in the Earth's magnetosphere are highly variable in time, and can produce different levels of surface charging on the materials that encase a satellite (Garrett 1981). A number of spacecraft anomalies, and even failures, have been identified as having occurred following many days of significantly elevated fluxes of several MeV energy electrons (Baker et al. 1987, 1996; Reeves et al. 1998). These very energetic particles can produce upsets and errors in space-borne 
Table 3 Categorization of components for space environment and their effects on spacecrafts (credit: Koskinen et al. 2001)

\begin{tabular}{|c|c|c|c|}
\hline $\begin{array}{l}\text { Components of the } \\
\text { space environment }\end{array}$ & Effects & System affected & Other problems \\
\hline Neutrals & $\begin{array}{l}\text { Drag } \\
\text { Oxidization } \\
\text { Contamination }\end{array}$ & $\begin{array}{l}\text { Orbit control } \\
\text { Temperature control } \\
\text { Shields }\end{array}$ & Glow \\
\hline Photons & $\begin{array}{l}\text { Heating } \\
\text { Photo-emission } \\
\text { Surface ageing } \\
\text { Background noise increase } \\
\text { Positive voltage }\end{array}$ & $\begin{array}{l}\text { Temperature control } \\
\text { Sensors }\end{array}$ & Contamination \\
\hline Plasmas & $\begin{array}{l}\text { Surface ageing } \\
\text { Current closure } \\
\text { Surface charging ESDs } \\
\text { EM noise }\end{array}$ & $\begin{array}{l}\text { High-voltage systems } \\
\text { Temperature control } \\
\text { RF systems }\end{array}$ & Contamination \\
\hline Particle radiation & $\begin{array}{l}\text { Ageing } \\
\text { Atom displacements } \\
\text { Internal charging ESDs } \\
\text { Background noise increase SEEs } \\
\text { DNA damage } \\
\text { Cell destruction }\end{array}$ & $\begin{array}{l}\text { Temperature control } \\
\text { Electronic components } \\
\text { Solar cells } \\
\text { Star trackers } \\
\text { Detector background } \\
\text { Living organisms }\end{array}$ & $\begin{array}{l}\text { Genetics, cancer } \\
\text { Death }\end{array}$ \\
\hline Meteoroids & $\begin{array}{l}\text { Impact } \\
\text { Induced neutral and plasma } \\
\text { environment }\end{array}$ & $\begin{array}{l}\text { Partial destruction } \\
\text { Attitude control } \\
\text { Coating erosion }\end{array}$ & $\begin{array}{l}\text { ESDs } \\
\text { Contamination }\end{array}$ \\
\hline Debris & $\begin{array}{l}\text { Impact } \\
\text { Induced neutral and plasma } \\
\text { environment }\end{array}$ & $\begin{array}{l}\text { Partial destruction } \\
\text { Attitude control } \\
\text { Coating erosion }\end{array}$ & $\begin{array}{l}\text { ESDs } \\
\text { Contamination }\end{array}$ \\
\hline Magnetic field & Local change & Magnetic attitude control & \\
\hline
\end{tabular}

electronic instruments (Todd 2000). There is no realistic shielding possible for systems in space that are bombarded by Galactic Cosmic Rays ( $\sim 1 \mathrm{GeV}$ and greater).

\subsection{Effects on Ground-Based Systems}

Large electric currents are continuously flowing in the magnetosphere and ionosphere; a disturbance in the solar wind changes to the current system. The variations of magnetospheric and ionospheric currents are seen as geomagnetic disturbances or storms at the Earth's surface. The auroral electrojet system is of particular importance concerning geomagnetic disturbances; similar effects are also experienced at lower latitudes (Rastogi 1999). According to electromagnetic theory, a geomagnetic variation is accompanied by a geoelectric field (Weaver 1994). The Earth consists of conducting material, so the geoelectric field drives currents within the Earth.

The geoelectric field implies the existence of voltages between different points on the Earth's surface which produce geomagnetically induced currents (GICs). Rapid temporal changes of the geomagnetic field produce GICs in technological conductor systems like electric power systems, oil and gas pipelines, telecommunications cables and railway equipment (Lanzerotti et al. 1999; Boteler 2003; Pirjola 2007; Huttunen et al. 2008).

Liu et al. (2009) have calculated GICs in mid- to low- latitudes power grids based on plane wave methods. However, this plane wave method is relatively rough, especially for 
applications to large areas and to sharp conductivity variations such as occur at coastal regions. More elaborate models should be studied, developed and used in further research.

\subsubsection{Power Line Systems}

The problems of geomagnetically induced currents in power lines and flowing in high voltage transformers have been recognized at least since the early 1970s (Albertson et al. 1974), but were brought into serious consideration by the widespread failure of the HydroQuebec power grid (Canada) resulting from the severe geomagnetic storm on March 13-14, 1989 (Blais and Metsa 1993; Bolduc et al. 1998). The estimated peak electrical power lost exceeded 20,000 MW and rendered millions of people problems for about $9 \mathrm{~h}$.

The problem occurs when quasi-DC (frequency range 0.01-0.0001 Hz) geomagnetically induced currents (GICs) flow between the high voltage lines and the Earth through transformer windings. The GICs can saturate the transformer core reducing their efficiency dramatically, perhaps damaging the transformer due to increased heating or triggering safety systems that remove the transformer from the circuit, and possibly creating knockon effects in the rest of the grid (Clark 2001; Crosby et al. 2006). It is not only the geoelectric field that dictates the GIC magnitude in power systems but also their geometrical and structural details have a significant influence (Molinski 2002; Trivedi et al. 2007). Usually GICs vary greatly from site to site in a power system, and model calculations may reveal those sites that are probably prone to the largest GIC magnitudes (Viljanen and Pirjola 1994; Wik et al. 2008). The severity of the effects on power grids also depends on the latitude of the grid, and on the conductivity of the underlying surrounding land or sea.

One of the most important factors determining the effects of GICs on a transmission system is transformer design, and hence space weather models can play an important role in specifying design parameters. An issue for further research is the effect of long term wear on transformers which have been exposed to saturating GICs over many years (Pirjola 2007; Liu et al. 2009).

\subsubsection{Pipe Line Systems}

Pipelines, particularly those that are buried or under water, may experience rapid corrosion due to GICs flowing through them. The problem is especially severe if they are not properly protected from corrosion. That is done by maintaining them at a negative electrical potential with respect to the ground (Clark 2001; Crosby et al. 2006). During a magnetic storm the electric field induced along the pipeline may counteract the applied potential, removing the corrosion protection and possible even accelerating corrosion (Pirjola et al. 1999; Trichtchenko and Boteler 2001).

To avoid corrosion, pipelines are equipped with a cathodic protection system (Von Baeckman et al. 1997). This tries to keep the pipeline at a negative potential with respect to the soil which inhibits current flow from the pipe to the soil. Different harmful processes may take place if the negative potential becomes too large; therefore the potential has to be carefully adjusted. This is an engineering solution to the space weather effect, to provide an active protection to cope with the most severe expected magnetic storms, rather than relying on a space weather prediction service (Viljanen et al. 2006). Space weather models may help in determining and specifying the design parameters. Reliable forecasts of geomagnetic activity will be useful in planning service schedules, when testing may be disrupted by geomagnetic activity. 


\subsubsection{Other Effects of Space Weather}

The first observations of space weather effects on technological systems were made in telegraph equipment more than 150 years ago (Barlow 1849). Many times since then, systems have suffered from overvoltages, interruptions in the operations and even fires caused by GICs flowing through the equipment (Boteler et al. 1998). Records of disturbances also offer a possibility of estimating the magnitude of geoelectric field by knowing the maximum overvoltage which the equipment can stand. It has been suggested that currents induced in railway signaling systems can be responsible for malfunctions of the system. During a magnetic storm in July 1982, such an effect made traffic lights turn red without any train coming, in Sweden (Wallerius 1982).

Submarine telephone cables lying on the ocean floors form a special category of systems affected by geomagnetic disturbances (Root 1979). Their length implies that the voltages induced on such cables during geomagnetic storms may reach some hundreds or even thousands of Volts, leading to possible problems (Lanzerotti et al. 1995). These voltages, which are also affected by the flow of seawater, are monitored on trans-Atlantic and transPacific cables and on some shorter ocean cables as well.

In exploration geophysics, magnetic surveys are conducted to obtain information about subsurface rocks. Measurements include temporal changes of the field arising from ionospheric and magnetospheric effects, so there is the problem of separating these variations from the desired spatial variations (Lanzerotti 1979). If surveys are made over small areas on land then the effect of the time dependent field can be removed by running a magnetometer at the base station and subtracting the observed field from the survey data. This method does not yield correct results if the survey region is far away from the base station and the region to be surveyed is quiet large. This is because the base data may not be representative of the external field variations all over the survey area.

If surveys from aircraft or ships are made, then care has to be taken that the survey is carried out during quiet periods. Because of cost implications, reliable forecasts of geomagnetic activity should be used in planning survey operations. As the intensity of the effect of a magnetic storm is latitude dependent, surveys in auroral regions are more affected than those in low latitude regions. It is clear that the issue here is one of designing systems to be free from geomagnetically induced electric fields. Space weather models may be of value in specifying system parameters during the design phase.

Another consequence of space weather is its effect on humans and biological systems in space and on aircraft (Baker et al. 2006). Solar proton events (SPEs) can knock electrons from cell molecules and damage them, especially from the skin, eye and blood-forming organs. These damaged cells are unrepairable (Crosby et al. 2006). If DNA (deoxyribo nucleic acid) is damaged then cell reproduction is hampered and even the effect could be passed to the next generations. Biological effects can also be in the form of severe burns, sterilization, cancer and damage to other organs.

Astronauts and cosmonauts on space missions are susceptible to the biological effects posed by energetic charged particles. Astronauts performing extra vehicular activity (EVA) in their spacesuits are less protected than those in their metallic space capsule or inside the Space Shuttle. A $10 \mathrm{~g} \mathrm{~cm}^{-2}$ aluminum shelter should provide adequate protection. Computer simulations show that inside a spacecraft an astronaut would absorb lethal doses of radiation in $10 \mathrm{~h}$ after the start of a major event (Turner 1996; Hanslmeier 2003). However, a number of studies have concluded that a properly shielded astronaut would not be exposed to radiation levels above the recommended limit (Wilson et al. 1997, 1999). Efforts are being made to study the response of exposure of biological material to the space 
environment and to measure the charged particles and solar extra-terrestrial radiation with a multi-channel dosimeter (Häder and Dachev 2003; Miroshnichenko 2008).

Galactic cosmic rays and transient solar proton events (SPEs) are the principal sources of natural radiation at aircraft altitudes $(\sim 10 \mathrm{~km})$. The radiation dose increases with altitude by a factor of $10^{3}$ from sea level up to $\sim 25 \mathrm{~km}$. On the ground, the dose is a factor of two higher near the poles than at the equator, and with solar cycle the value is similar (Shea and Smart 2000a, b). The crew and passengers on board aircraft flying at greater altitudes and on polar routes are more susceptible to radiation hazards than those flying at lower altitudes on non-polar routes (Jones et al. 2001). With increasing air traffic, aircraft are bound to fly at higher altitudes and, as a consequence, the possibility of harmful effects of radiation will be enhanced.

The variations of cosmic radiation at aircraft altitudes are being studied jointly by the University College London's Mullard Space Science Laboratory and Virgin Atlantic Airways. In flight, measurements are being made using Tissue Equivalent Proportional Counters. Details are available on the website http://www.mssl.ucl.ac.uk/cosrad/index.htm.

\section{Predictability of Space Weather}

Forecasting space weather is the specification of the state of the space environment at a future time; it is a major challenge (Singer et al. 2001; Schwenn et al. 2005; Weigel et al. 2006). This is because, in addition to formulating and implementing a test of a model or theory, it is burdened with the complications of issuing a predictive statement with limited information and in advance of the event (Vassiliadis 2007). The trustworthiness and accuracy of space weather forecasting, even for large solar wind events, i.e. flares and CMEs, and their impacts are still poor (Watermann et al. 2009a, b). Space weather predictions involve forecasting the time of occurrence and arrival at the Earth of CMEs at the outer boundary of the magnetosphere and their interaction with it. They depend on our understanding of all the relevant phenomena, their consequences and the development of technological capabilities to monitor precisely their features. Thus, one challenge of making predictions of space weather depends strongly on how the prediction will be used. The problem is complicated by the fact that a device in space can be affected in many different ways.

Solar wind effects on the magnetosphere range from a compression of the magnetopause caused by solar wind dynamic pressure to heating of neutral atmosphere through processes including Joule heating. The impact of violent solar wind perturbations on geospace is also felt through geomagnetic storms (large deviations of the magnitude and orientation of geomagnetic field), particle storms (enhanced radiation levels in the magnetosphere) and ionospheric storms (increased and highly variable ionization of the upper atmosphere and sometimes even the middle atmosphere). Geomagnetic storms during the later declining and minimum phases of the solar cycle are caused mostly by CIRs whereas intense storms at solar maximum and the early declining phases of the cycle are caused by ICMEs (Cid et al. 2004; Denton et al. 2006). Borovsky and Denton (2006) have listed significant differences between CME driven and CIR driven storms and their effects on space-borne and ground based technological systems. They have also discussed the influence of storms on magnetospheric convection and energetic charged particle fluxes.

The plasma particle distribution in the cusp region and outer radiation belt tend to vary in a relatively coherent way under the influence of major external drivers like solar wind streams and CMEs. Thus it is possible to use the overall behavior of the outer zone electron radiation belt population and the day to day variations for a single satellite or a few 
satellites only. Specification models that use magnetic activity indices can characterize the state of the outer radiation belt (Moorer and Baker 2001). Prediction schemes have been developed based on a combination of earlier values of radiation belt fluxes, and past and present solar wind parameters (Watermann et al. 2009a, b).

Coronal mass ejections are routinely recorded by the SOHO instruments (Yashiro et al. 2004) and important information about CMEs, including their travel direction, determined from solar coronagraph data (Yashiro et al. 2004) made available to the science community through the website: http://cdaw.grfc.nasa.gov/CME_list/. Space weather effects will be produced by those CMEs events that propagate in a direction which may lead to an encounter with the near-Earth space environment. Only such events require space weather warnings because they can produce space weather effects. CME events that are not propagating towards the Earth will not interact with the Earth's space environment and hence in those cases space weather warnings are not required.

The prediction of geomagnetic activity comes down to the prediction of the state of the solar wind at the magnetopause, which depends on solar flare eruptions, CMEs, halo CMEs and SEP events. No model currently exists that can predict the onset of CMEs at the Sun (Messerotti 2008) based on solar parameters. We can only track bipolar regions as potential sources of CMEs from the Sun's East-to-West limb and monitor CME onset in the white light and the source region activity at EUV wavelengths (Tripathi et al. 2004). It is difficult to measure the real speed of CMEs and halo CMEs because of projection effects (Cremades and Bothmer 2004). However, to do that is essential to forecasting its arrival at the Earth's orbit. Also it is difficult to quantify the geoeffective (in terms of severity of geomagnetic activity) parameters of CMEs (i.e. their speed, intensity and duration of associated southward directed magnetic field) in advance.

Several authors have used the plane-of-sky speed for forecasting (Gopalswami et al. 2001; Cane and Richardson 2003; Michalek et al. 2003); the plane-of-sky speed is oriented perpendicular to the Sun-Earth line. Schwenn et al. (2005) have developed an empirical relationship between the CMEs travel time to $1 \mathrm{AU}$ and its expansion speed in the SOHO coronagraph's field of view. Their empirical formula yields arrival times with a 95\% error margin of $24 \mathrm{~h}$. For a CME observed near the Sun, it takes about 1-4 days for the associated ICME to reach 1 AU. The observed error in arrival time of an ICME may be due to travel through a very different type of ambient solar wind. Also it might have undergone acceleration or deceleration depending on the relative solar wind speeds.

The majority of intense geomagnetic storms $\left(D_{\mathrm{st}} \leq-100 \mathrm{nT}\right)$ are generated by halo CMEs (Cid et al. 2004), but many frontside halo CMEs are not followed by intense storms. Hence forecasting magnetic storms only on the basis of their occurrence will lead to false alarms (St Cyr et al. 2000). Akasofu and Fry (1986) analyzed more than 200 halo CMEs and showed that only one event originating at longitude larger than $30^{\circ}$ from the solar central meridian resulted in a magnetic storm with $D_{\text {st }} \leq-200 \mathrm{nT}$. Gopalswamy et al. (2007) analyzed 378 halo CMEs and proposed that halos originating at longitudes $>45^{\circ}$ from the central meridian could generally produce moderate storms $\left(-50 \mathrm{nT} \geq D_{\mathrm{st}} \geq\right.$ $-100 \mathrm{nT}$ ), whereas those originating closer to disk centre (longitudes $<45^{\circ}$ ) may generate stronger storms $\left(D_{\mathrm{st}} \leq-100 \mathrm{nT}\right)$. This was considered to be a consequence of the angular width of a typical CME being around $50-60^{\circ}$ (St Cyr et al. 2000).

Recently Rodriguez et al. (2009) presented an analysis of three front side full halo CMEs, which have non typical geomagnetic responses. All of them had plane-of-sky speeds higher than 1,100 km/s and two of them (observed on September 12, 2000 and June $6,2000)$ originated closer to the central meridian but resulted in a weaker geomagnetic response $\left(D_{\mathrm{st}}=-34 \mathrm{nT}\right.$ and $-90 \mathrm{nT}$, respectively). The third one was initiated farther 
away from the disk (Observed on March 4, 2000) and produced the strongest geomagnetic disturbance $\left(D_{\mathrm{st}}=-288 \mathrm{nT}\right)$. This unusual behavior is explained using measurements from the Advanced Composition Explorer (ACE) spacecraft positioned at the L1 point (1.5 million km upstream of Earth) (Skone and Cannon 1998). The halo CME (September $12,2000)$ was highly asymmetric with its bulk going towards the South. The associated post-shock solar wind could not produce strong $-B_{\mathrm{z}}$, leading to only a moderate geomagnetic storm $\left(D_{\mathrm{st}}=-39 \mathrm{nT}\right)$.

This result is in agreement with that of Moon et al. (2005) who concluded that the mass distribution in the CME correlates well with the geoeffectiveness of the CME. The ICME corresponding to the halo CME of June 6, 2000 had a $B_{\mathrm{z}}=-20 \mathrm{nT}$ in the post-shock sheath region but the duration of this enhancement was too short to produce a strong geomagnetic storm; there was only a moderate storm with $D_{\text {st }} \sim-90 \mathrm{nT}$. This shows that enhanced southward magnetic field for a long duration can produce a strong geomagnetic storm. In the case of the halo CME on June 6, 2000, the strong shock arrived at the Earth, produced strong and long lasting negative $B_{\mathrm{z}}$ and resulted in an intense geomagnetic storm with the minimum $D_{\text {st }}=-288 \mathrm{nT}$. In this case also, an asymmetric mass distribution was observed. Rodriguez et al. (2009) proposed that this event might have been the result of a strong and fast CME overtaking a previous slower one. They also discussed another possibility, i.e., the strong compression at the eastern flank of the sheath and eastward deflection of the fast ICME by the magnetic tension force from the IMF in the ambient slow solar wind flow might have led to strong geoeffectiveness. These discussions show that forecasting the geoeffectiveness of a CME is still far away and requires a more precise understanding of the physical phenomena involved.

Information about CMEs, their velocity and their place of origin on the solar surface enables geomagnetic activity to be forecast 1-3 days in advance (Srivastava 2005; Gleisner and Watermann 2006a, b), the source of the most severe geomagnetic disturbances being CMEs with the highest velocity (Tsurutani 2001; Kim et al. 2005). However, the effects in the near-Earth environment critically depend on whether the interplanetary magnetic field in the post-shock solar wind rotates from North to South or vice versa. From solar observations alone, it is impossible to detect either the solar wind speed in interplanetary space or the structure of the magnetic field, and hence the intensity and duration of the geoeffective southward field direction (Bothmer and Rust 1997). All intense magnetic storms $\left(D_{\mathrm{st}} \leq-100 \mathrm{nT}\right)$ of the $23 \mathrm{rd}$ solar cycle occurred under long-lasting southward IMF conditions (Echer et al. 2008). The most geoeffective interplanetary structures were identified as MCs driving fast shocks, sheath fields sandwiched between ICMEs and associated shocks, or combined ICME, sheath and MC fields and CIRs (Echer et al. 2008).

The SMEI (Solar Mass Ejection Imager) provides better opportunity for forecasting the occurrence of storms by tracking CMEs from near the Sun to Earth (Webb et al. 2009). It can detect a number of geoeffective ICMEs at elongations of $20-30^{\circ}$ from the centre of the Sun. Webb et al. (2009) could produce near-real-time predictions using LASCO and SMEI data for two storms. They have claimed that, on average, SMEI is capable of the first detection of a geoeffective ICME about 1 day in advance. Considering $6 \mathrm{~h}$ to be the time required by a forecaster to run a prediction program for the arrival time after data from SMEI are used, a prediction lead time of $\sim 18 \mathrm{~h}$ can be achieved. Recently Davis et al. (2009) analyzed the CME imaged by Heliospheric Images (HI) onboard the twin STEREO spacecraft during December 15-19, 2008, and showed that a CME impact could have been forecast at least $24 \mathrm{~h}$ in advance. Their analysis showed that the stereoscopic imaging could predict the arrival time at the position of the ACE spacecraft and Earth to an accuracy of a few hours. In the analyzed CME, the shock front was weak and no SEPs were 
observed, except a few energetic helium ions. STEREO observations could provide forecasting of the timing and potential fluxes at least $24 \mathrm{~h}$ in advance, with better precision than for the earlier cases (Webb et al. 2009).

Webb et al. (2009) have discussed the prediction of ICMEs using SMEI observations and the HAFv2 (Hakamada- Akasofu-Fry version 2) 3-D solar wind model (Hakamada and Akasofu 1982; Fry et al. 2001, 2003). They showed that the model could predict the ICME properties with reasonable accuracy. The HAFv2 is a kinematic model which predicts solar wind conditions such as speed, density, dynamic pressure and interplanetary magnetic field at the Earth and elsewhere in the solar system based on observations at the Sun (Webb et al. 2009). Figure 11 represents the forecast of ecliptic plane IMF showing the shock propagating through a pre-existing CIR based on SMEI observations of an Earthward directed halo ICME on May 29, 2003 and the HAFv2 model (Sun et al. 2008). The new results from the STEREO mission (Russell 2008) will help researchers to prove or reject existing theories on CME initiation, three dimensional structure evolution and propagation. Hence, the results from the mission will be a quantum jump for space weather research and predictions.

Hathaway and Wilson (2004) have shown the strong positive correlation between sunspot numbers and $10.7 \mathrm{~cm}$ radio flux, total irradiance, X-ray flares, sunspot area, the base-line level of geomagnetic activity and the flux of galactic cosmic rays. Recently, Watari (2008) suggested a good negative correlation (correlation coefficient $\sim-0.661$ ) between solar cycle length and the maximum monthly sunspot number of the next cycle. On the basis of these correlations, space weather parameters may be predicted. The predicted maximum sunspot number for the 24th cycle would be about 101 and the next solar maximum would occur around July 2012; the length of the cycle would be 11 years.

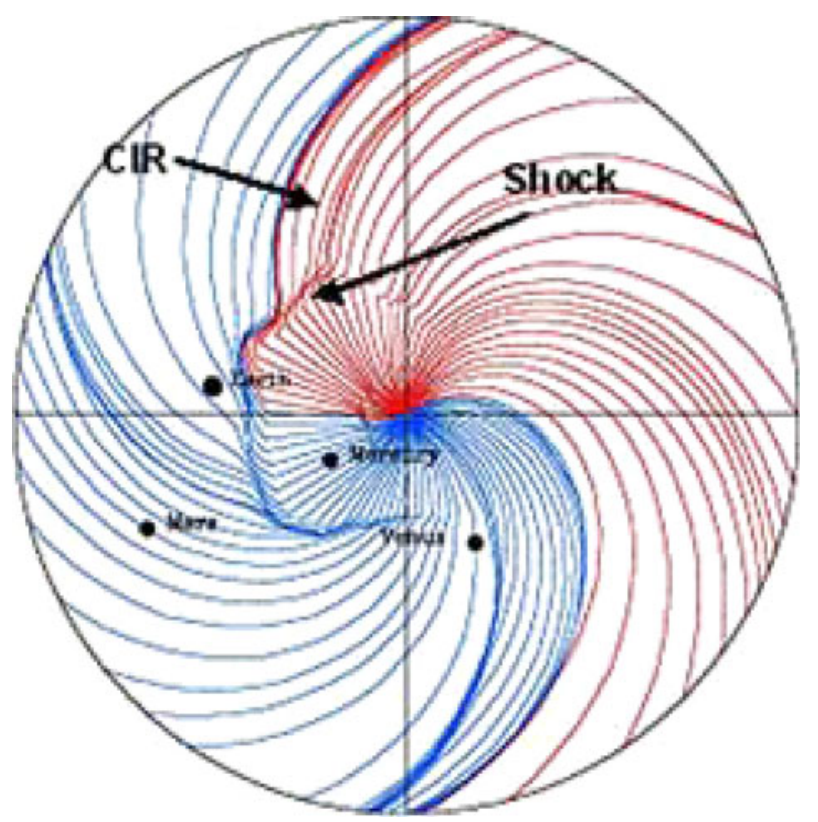

Fig. 11 Simulated results of IMF in ecliptic plane showing the shock propagating through a pre-existing CIR (credit: Webb et al. 2009) 
Recently, Valach et al. (2009), using an artificial neural network model, has tried to predict geomagnetic activity on the basis of solar energetic particle flux enhancements. The model is based on the scheme proposed by Valach et al. (2007). Data concerning X-ray flares and solar radio bursts (type II giving the signature of shock initiation in the solar corona and type IV representing material moving upward in the corona) are used as input parameters for the neural network. They showed that inclusion of information about high energy proton flux data as additional input improves the forecasts. Analysis showed about $51 \%$ forecast response (Valach et al. 2009).

Having the ability to predict such events today should save society hundreds of millions of dollars a year (Baker 2009). As the modern world becomes ever more dependent on technology that can be vulnerable to changes in the near-Earth space environment, the need for accurate forecasting only increases. Accordingly various models are being developed for the study and forecasting of space weather phenomena. Messerotti et al. (2009) have reviewed the attempts made to model sunspots, solar radio bursts, solar flares, coronal holes, coronal mass ejections and solar wind. All these phenomena are governed by the distribution and variability of solar magnetic field. Precise measurements or reconstructions of magnetic field over the whole solar surface have not yet been possible (Messerotti et al. 2009). Precise prediction of space weather requires precise knowledge of the solar magnetic field. Further complications in developing suitable models arise from the stochastic and chaotic behavior of solar plasma processes and the vast ranges of temporal, spatial and energetic scales involved (Watermann et al. 2009a, b).

Groups working at the Center for Space Environment Modeling (CSEM), University of Michigan, and the center for Integrated Space Weather Modeling (CISM), Boston University, USA, are engaged in developing comprehensive modules to study events from the solar corona to the Earth's atmosphere. The details of the models are available at the websites http://butch.engin.umich.edu/ and http://www.bu.edu/cism/. The CSEM developed a Space Weather Modeling Framework (SWFM) modularized scheme (Tóth et al. 2005). It contains various versions and combinations of modules for the different phenomena involved in space weather studies. Similarly CISM also contains different modules. Each module can run separately or in a coupled mode, depending upon the requirements of the phenomena to be studied. Despite the various models currently available, not a single model can predict the onset of a CME, its evolution and time of arrival at the magnetopause, and its consequences as a space weather event.

\section{Summary}

We have discussed the physics, the effects and predictability of space weather, a topic of interest to present day society, which is increasingly relying on space-based infrastructure for communications and national security. There is a need to improve public awareness of the risks which space weather poses. Additionally ambitious programs are being talked about, such as developing colonies on the Moon and Mars, harvesting solar energy in space, harvesting minerals and other precious elements available on other planets or asteroids.

We have briefly discussed the solar wind, solar flares and their interaction with the Earth's magnetosphere. The present knowledge of CMEs including halo CMEs, MCs and CIRs are reviewed because of their important role in the space weather. What is known of their origin, propagation in interplanetary space and their effect in the magnetosphere and atmosphere of the Earth have been considered. Out of various theories proposed for the 
generation of CMEs, the magnetic flux-rope theory seems to explain some features of CMEs. Improvements in knowledge of the photospheric magnetic field structure, and of the connection between small scale and large scale structures are required to understand CME eruption. Understanding the mechanisms that lead to the eruption of CMEs will be essential for making accurate predictions at the Earth. There is a question about the root of magnetic clouds, as to whether they are connected to the solar surface or not. However, many more efforts are required through modeling and refinement of observation techniques to understand the generation and propagation mechanisms of CMEs and ICMEs. The prediction of the geoeffectiveness of ICMEs is at present at a developing stage, because efforts in this field are dependent upon the quality and quantity of observations as well as on the understanding of physical phenomena taking place at the solar surface. Results from the recent STEREO mission may improve the situation in regard to the relevant data.

The solar wind plasma and IMF control the formation, shape and size and location of the northern and southern cusps on the magnetopause, which are the main places where plasma directly enters the magnetosphere. Observations by the RAPID instruments aboard the Cluster spacecraft shed light on high latitude reconnection when the IMF is northward. However, in order to understand convection in the cusp regions and their dynamics, which are linked with the interplanetary conditions, require many more experimental and theoretical/simulation studies.

The effects of space weather on communications, positioning and navigation, electronic instruments aboard satellites, wireless systems, power line systems, pipelines, and biological systems (astronauts, airline crew and passengers) have been discussed. Finally, we have attempted to present current possibilities for the prediction of space weather causes and their consequences. Reliable and accurate prediction requires a precise understanding of the many physical processes involved; here relevant measurements are being used as input parameters. Measurements of solar wind and CME/ICME parameters from space are helpful in improving our understanding of the basic processes, as well as providing input parameters 1 day in advance. Considering the time required for computation, the prediction of the CME/ICMEs arriving at the Earth could be made at least $18 \mathrm{~h}$ in advance.

Many sophisticated modeling efforts of the Sun-Earth system are currently underway, but there is a serious need for more detailed models and improved predictions of intense solar activity because of its severe impact on today's human society.

Acknowledgments AKS is grateful to the Department of Science and Technology (DST), Government of India for providing financial support as a research project (File no. SR/S4/AS/261/06). AKS, DS are also thankful to Indian Space Research Organisation (ISRO) for partial financial support under CAWSESS program. RPS acknowledges the facilities provided by the Head, Department of Physics, BHU, Varanasi. The authors express their thanks to the referee for many important comments and to Prof M. J. Rycroft's suggestions and editorial attention, both of which have greatly improved the quality and the content of the paper.

\section{References}

Abel B, Thorne RM (1998) Electron scattering loss in Earth's inner magnetosphere 1: dominant physical processes. J Geophys Res 103:2385-2395

Afraimovich EL, Zherebtsov GA, Smolkov GYA (2007) Total failure of GPS during a solar flare on December 6 2006. Dokl Earth Sci 417:1231. doi:10.1134/S1028334X07080223

Afraimovich EL, Demyanov VV, Ishin AB, Smolkov GYA (2008) Powerful solar radio burst as a global and free tool for testing satellite broadband radio systems, including GPS-GLONASS-GALILEO. J Atmos Solar Terr Phys 70:1985-1994. doi:10.1016/j.jastp.2008.09.008 
Akasofu SI (1968) Polar and magnetospheric substorms. D. Reidel, Dordrecht, p 280

Akasofu SI (1981) Energy coupling between the solar wind and the magnetosphere. Space Sci Rev 28:121-190

Akasofu SI (2007) Long-standing unsolved problems in solar terrestrial physics. IEEE Trans Plasma Sci 35:751-758

Akasofu SI, Fry CF (1986) A first generation numerical geomagnetic storm prediction scheme. Planet Space Sci 34:77-79. doi:10.1016/0032-0633(86)90105-4

Albert JM (2003) Evaluation of quasi-linear diffusion coefficients for EMIC waves in a multispecies plasma. J Geophys Res 108:1249. doi:10.1029/2002JA009792

Albertson VD, Thorson JM Jr, Miske SA Jr (1974) The effects of geomagnetic storms on electrical power systems. IEEE Trans Power Apparatus Syst PAS-93(4):1031-1044

Allen J, Wilkinson D (1993) Solar-terrestrial activity affecting systems in space and on earth, solar-terrestrial predictions IV. In: Proceedings of a workshop at Ottawa, Canada, 18-22 May, 1992, vol 1. Published by NOAA Environment Research Laboratory, Boulder, p 75

Alves MV, Echer E, Gonzalez WD (2006) Geoeffectiveness of corotating interaction regions as measured by $D_{\text {st }}$ index. J Geophys Res 111:A07S05. doi:10.1029/2005JA011379

Amari T, Luciani JF, Aly JJ (2004) Coronal magnetohydrodynamic evolution driven by subphotospheric conditions. Astrophys J 615:L165-L168

Amsler C et al (2008) Review of particle physics: gauge and higgs bosons. Phys Lett B 667:1

Anderson CW, Lanzerotti LJ, Maclennan CG (1974) Outage of the L-4 system and geomagnetic disturbances on August 4, 1972. Bell Syst Tech J 53:1817

Antonova AE, Gubar YuI, Kropotlin AP (2000) Energetic particle population in the high-latitude geomagnetosphere. Phys Chem Earth (C) 25:47-50

Aschwanden MJ (2004) Physics of the solar corona. Springer, Berlin

Avakyan SV (2008) The physics of Sun-Earth coupling: results, problems, and new approaches. Geomagn Aeron 48:4

Axford WI, Hines CO (1961) A unifying theory of high-latitude geophysical phenomena and geomagnetic storms. Can J Phys 39:1433

Bailey GJ, Denton MH, Heelis RA, Venkatraman S (2000) A modeling study of the latitudinal variations in the nighttime plasma temperatures of the equatorial topside ionosphere during northern winter at solar maximum. Ann Geophys 18:1435-1446. doi:10.1007/s00585-000-1435-6

Baker DN (1998) What is space weather? Adv Space Res 23:1-7

Baker DN (2009) What does space weather cost modern societies? Space Weather 7:S02003. doi: $10.1029 / 2009$ SW000465

Baker DN, Blake JB, Gorney DJ, Higbie PR, Klebesadel RW, King JH (1987) Highly relativistic magnetospheric electrons: a role in coupling to the middle atmosphere? Geophys Res Lett 14:1027

Baker DN, Pulkkinen TI, Angelopoulos V, Baumjohann W, McPherron RL (1996) The neutral line model of substorms: past results and present view. J Geophys Res 101:12975

Baker DN, Kanekal SG, Li X, Monk SP, Goldstein J, Burch JL (2004) An extreme distortion of the Van Allen Belt arising from the 'halloween' solar storm in 2003. Nature 432:878-881

Baker DN, Barby LA, Curtis S, Jokipii JR, Lewis WS, Miller J, Schimmerling W, Singer HJ, Townsend LW, Turner RE, Zurbuchen TH (2006) Space radiation hazard and the vision for space exploration: report of a workshop. The National Academy Press, Washington

Baker DN, Wiltberger MJ, Weigel RS, Elkington SR (2007) Present status and future challenges of modeling the Sun-Earth end-to-end system. J Atmos Solar Terr Phys 69:3-17

Balasubrahmanyan VK, Serlemitsos AT (1974) Solar energetic particle event with He-3/He-4 greater than 1. Nature 252:460-462

Balogh A, Lanzerotti LJ, Suess ST (2008) The heliosphere through the solar activity cycle. Springer, Berlin

Balsiger H, Eberhardt P, Geiss J, Young DT (1980) Magnetic storm injection of 0.9-16 keV/e solar and terrestrial ions into the high altitude magnetosphere. J Geophys Res 85:1649

Barbieri LP, Mahmot RE (2004) October-November 2003's space weather and operations lessons learned. Space Weather 2:S09002. doi:10.1029/2004SW000064

Barlow WH (1849) On the spontaneous electrical currents observed in wires of the electrical telegraph. Phil Trans R Soc Lond 139:61

Barnes G, Leka KD, Schumer EA, Della-Rose DJ (2007) Probabilistic forecasting of solar flares from vector magnetogram data. Space Weather 5:S09002. doi:10.1029/2007SW000317

Barron WR, Cliver EW, Cronin JP, Guidice DA (1985) Solar radio emission. In: Jura AS (ed) Handbook of geophysics and the space environment, Chap. 11, AFGL, USAF

Bastian TS, Benz AO, Gary DE (1998) Radio emission from solar flares. Ann Rev Astron Astrophys 36:131 
Bastian TS, Pick M, Kerdraon A, Maia D, Vourlidas A (2001) The coronal mass ejection of 1998 April 20: direct imaging at radio wavelengths. Astrophys J 558:65-69

Baumjohann W, Pellinen RJ, Opgenoorth HJ, Nielsen E (1981) Joint two dimensional observations of ground magnetic and ionospheric electric fields associated with auroral zone currents: current systems associated with the local break-ups. Planet Space Sci 29:431-447

Bertaux JL, Le Texier H, Goutail F, Lallement R, Kockarts G (1989) Lyman alpha observations of geocoronal and interplanetary hydrogen from Spacelab-1: exospheric temperature and density and hot emission. Ann Geophys 7:549-564

Bieber JW, Evenson P, Dröge W, Pyle R, Ruffolo D, Rujiwarodom M, Tooprakai P (2004) Spaceship Earth observations of the Easter 2001 solar particle event. Astrophys J 601:L103-L106

Bieber JW, Clem J, Evenson P, Pyle R, Ruffolo D, Saiz A (2005) Relativistic solar neutrons and protons on 28 October 2003. Geophys Res Lett 32:L03S02

Biermann L, Haxel O, Schlüter A (1951) Neutrale ultrastrahlungen von der Sonne, Zs. Naturforschung, $6 \mathrm{a}: 47$

Bilitza D (2001) International reference ionosphere 2000. Radio Sci 36:261-275

Blais G, Metsa P (1993) Operating the hydro-Quebec grid under magnetic storm conditions since the storm of 13 March, 1989, in solar-terrestrial prediction IV. In: Hruska J, Shea MA, Smart DF, Heckman G (eds) Proceedings of a workshop at Ottawa, Canada, May 18-20, 1992, NOAA

Blake B (1999) Comment on 'cusp: a new acceleration region of the magnetosphere', by J Chen et al. Czech J Phys 49:675

Blake JB, Kolasinski WA, Fillius RW, Mullen EG (1992) Injection of electrons and protons with energies of tens of MeV into L less than 3 on 24 March 1991. Geophys Res Lett 19:821-824

Blanc M, Horwitz JL, Blake JB, Daglis IA, Lemaire JF, Moldwin MB, Orsini S, Thorne RM, Wolfe RA (1999) Source and loss processes in the inner magnetosphere. In: Hultqvist B, Øieroset M, Paschmann G, Treumann R (eds) Magnetospheric plasma sources and losses, Space Science Series of the International Space Science Institute, vol 6. Kluwer, Dordrecht, pp 137-206

Bolduc L, Langlois P, Boteler D, Pirjola R (1998) A study of geoelectromagnetic disturbances in Québec, 1. General results. IEEE Trans Power Deliv 13:1251

Borovsky JE, Denton MH (2006) Differences between CME-driven storms and CIR-driven storms. J Geophys Res 111:A07S08. doi:10.1029/2005JA011447

Borovsky JE, Denton MH (2009) Relativistic electron dropouts and recovery: a superposed epoch study of the magnetosphere and the solar wind. J Geophys Res 114:A02201. doi:10.1029/2008-A013128

Boscher D, Bougeret JL, Breton J, Lantos P, Prado JY, Romero M (1998) Météorologie de l'environnement spatial : rapport final du groupe d'évaluation des besoins”, RF CNES/DP/CM 98-252, octobre 1998. (English version provided by ESA)

Boteler DH (2003) Geomagnetic hazards to conducting networks. Nat Hazards 28:537-561

Boteler DH, Pirjola RJ, Nevanlinna H (1998) The effects of geomagnetic disturbances on electrical systems at the earth's surface. Adv Space Res 26:17-27

Bothmer V, Daglis IA (eds) (2007) Space weather: physics and effects. Springer, Dordrecht

Bothmer V, Rust DM (1997) The field configuration of magnetic clouds and the solar cycle. In: Crooker N, Joselyn JA, Feynman J (eds) Coronal mass ejections, vol 99 of Geophysical Monograph, pp 137-146. American Geophysical Union, Washington

Bothmer V, Schwenn R (1998) The structure and origin of magnetic clouds in the solar wind. Ann Geophys 16:1-24

Bothmer V, Zhukov A (2006) The Sun as the prime source of space weather. In: Bothmer V, Daglis Y (eds) Space weather-physics and effects. Springer/Praxis, Berlin, pp 31-102 2006

Bourdarie S, Bourrieau J (1999) Evaluation des taux d'événements singuliers induits par les protons piégés-Tirs AR5-XMM, CELESTRI et GTO+, RF/473900 ONERA/DESP, 1999

Bourrieau J, David JP, Levy L (1996) Environnement spatial, Notes de cours", SUPAERO course

Brückner GE (1974) The Behaviour of the outer solar corona $\left(3 R_{\odot}\right.$ to $10 R_{\odot}$ during a large solar flare observed from OSO-7 in White Light. In: Newkirk G Jr (ed) Coronal disturbances IAU Symposium no. 57, held at Surfers Paradise, Queensland, 7-11 Sep 1973, pp 333-334. Reidel, Dordrecht

Brückner GE, Howard RA, Koomen MJ, Korendyke CM, Michels DJ, Moses JD, Socker DG, Dere KP, Lamy PL, Llebaria A, Bout MV, Schwenn R, Simnett GM, Bedford DK, Eyles CJ (1995) The large angle spectroscopic coronagraph (LASCO). Sol Phys 162:357-402. doi:10.1007/BF00733434

Bučík R, Kudela K, Kuznetsov SN (2006) Satellite observations of lightning-induced hard X-ray flux enhancements in the conjugate region. Ann Geophys 24:1969-1976

Burkepile JT, Hundhausen AJ, Stanger AL, St Cyr OC, Seiden JA (2004) Role of projection effects on solar coronal mass ejection properties: 1. A study of CMEs associated with limb activity. J Geophys Res 109:3103 
Burlaga LF (1991) Magnetic clouds. In: Schwenn R, Marsch E (eds) Physics of the inner heliosphere, vol II: particles, waves and turbulence, vol 20 of physics and chemistry in space, pp 1-22. Springer, Berlin

Burlaga LF, Sittler E, Mariani F, Schwenn R (1981) Magnetic loop behind an interplanetary shock: Voyager, Helios, and IMP 8 observations. J Geophys Res 86:6673-6684

Burton RK, McPherron RL, Russell CT (1975) An empirical relationship between interplanetary conditions and $D_{\text {st. }}$ J Geophys Res 80:4204-4214. doi:10.1029/JA080i031p04204

Cane HV, Erickson WC (2005) Solar type II radio bursts and IP type II events. Astrophys J 623:1180-1194

Cane HV, Richardson IG (2003) Interplanetary coronal mass ejections in the near-Earth solar wind during 1996-2002. J Geophys Res 108:1156

Cannon PS (1994a) Propagation in the ionosphere (A). In: Propagation modelling and decision aids for communications, radar and navigation systems, pp 1A1-1A10. NATO-AGARD

Cannon PS (1994b) Propagation in the ionosphere (A). In: Propagation modelling and decision aids for communications, radar and navigation systems, pp 1B1-1B17. NATO-AGARD

Cargill PJ (2009) Coronal magnetism: difficulties and prospects. Space Sci Rev 144:413-421. doi: $10.1007 / \mathrm{s} 11214-008-9446-9$

Cargill PJ, Harra LK (2007) Coronal mass ejection. In: Kamide Y, Chian A (eds) Handbook of the solar terrestrial environment. Springer, Berlin, pp 118-132

Carlowicz MJ, Lopez RE (2002) Storms from the Sun. Joseph Henry Press, Washington

Carrington RC (1859) Description of a 'singular appearance' seen in the Sun on September 1, 1859. Mon Not R Astron Soc 20:13-15

Celsius A, Hiorter OP (1747) Om Magnet-nalens Atskillige andreingar. Kongle Swen Wetenskaps Acad Handlingar, pp 27-43

Cerruti AP, Kintner PM, Gary DE, Lanzerotti LJ, De Paula ER, Vo HB (2006) Observed solar radio burst effects on GPS/wide area augmentation system carrier-to-noise ratio. Space Weather 4:S10006. doi: $10.1029 / 2006$ SW000254

Chan Y, Reeves GD, Friedel RHW (2007) The energization of relativistic electrons in the outer Van Allen radiation belt. Nature Phys 3:614

Chang SW, Scudder JD, Fennell JF, Friedl R, Lepping RP, Russell CT, Trattner KJ, Fuselier SA, Peterson WK, Spence HE (2000) Energetic magnetosheath ions connected to the earth's bow shock: possible source of cusp energetic ions. J Geophys Res 105:5471

Chen J, Fritz TA (1999) Features of the cusp energetic particle events. Adv Space Res 24:103-107

Chen J, Fritz TA (2001) Energetic oxygen ions of ionospheric origin observed in the Cusp. Geophys Res Lett 28:1459-1462

Chen J, Fritz TA (2002a) Multiple spacecraft observations of energetic ions during a major geomagnetic storm. Adv Space Res 30:1749-1756

Chen J, Fritz TA (2002b) The global significance of the CEP events. In: Wang HN, Xu RL (eds) Solarterrestrial magnetic activity and space environment. COSPAR Colloquia Series, vol 14, pp 239-249

Chen J, Fritz TA, Sheldon RB, Spence HE, Spjeldvik WN, Fennell JF, Livi S, Russell CT, Pickett JS, Gurnett DA (1998) Cusp energetic particle events: implications for a major acceleration region of the magnetosphere. J Geophys Res 103:69-78

Chen Z, Gao Y, Liu Z (2005) Evaluation of solar radio bursts' effect on GPS receiver signal tracking within international GPS service network. Radio Sci 40:RS3012. doi:10.1029/2004RS003066

Chupp EL (1988) Solar neutron observations and their relation to solar flare acceleration problems. Solar Phys 118:137

Chupp EL, Forrest DJ, Ryan JM, Heslin J, Reppin C, Pinkau K, Kanbach G, Rieger E, Share GH (1982) A direct observation of solar neutrons following the 01:18 UT flare on 1982 June 3. Astrophys J 318:913-925

Chupp EL, Debrunner H, Flückiger EO, Forrest DJ, Golliez F, Kanbach G, Vestrand WT, Cooper J, Share G (1987) Solar neutron emissivity during the large flare on 1982 June 3. Astrophys J 318:913

Cid C, Hildago MA, Nieves-Chinchilla T, Sequeiros J, Viñas AF (2002) Plasma and magnetic field inside magnetic clouds: a global study. Sol Phys 207:187-198

Cid C, Hildago MA, Saiz E, Cerrato Y, Sequeiros J (2004) Sources of intense geomagnetic storms over the rise of solar cycle 23. Sol Phys 223:231-243

Clark TDG (2001) A review of effects of space weather on ground based technology. In: Proceedings of the workshop on space weather, ESTEC

Cliver EW, Svalgaard L (2004) The 1859 solar-terrestrial disturbance and the current limits of extreme space weather activity. Sol Phys 224:407-422

Cliver EW, Webb DF, Howard RA (1999) On the origin of solar metric type II bursts. Solar Phys 187:89-114 
Cliver EW, Nitta NV, Thompson BJ, Zhang J (2004) Coronal shocks of November 1997 revisited: the CME Type II timing problem. Sol Phys 225:105-139

Colak T, Qahwaji R (2009) Automated solar activity prediction: a hybrid computer platform using machine learning and solar imaging for automated prediction of solar flares. Space Weather 7:S06001. doi: 10.1029/2008SW000401

Contarino L, Zuccarello F, Romano P, Spadaro D, Guglielmino SL, Battiato V (2009) Flare forecasting based on sunspot-groups characteristics. Acta Geophys 57:52-63. doi:10.2478/s11600-008-0067-1

Cooke DJ, Humble JE, Shea MA, Smart DF, Lund N, Rasmussen IL, Byrnak B, Goret P, Petrou N (1991) On cosmic-ray cutoff terminology. Il Nuovo Cimento 14C:213-234

Cremades H, Bothmer V (2004) On the three dimensional configuration of coronal mass ejections. Astron Astrophys 422:307-322

Cremades H, Bothmer V, Tripathi D (2006) Properties of structured coronal mass ejections in solar cycle 23. Adv Space Res 38:461-465

Cronin J, Gaisser TK, Swordy SP (1997) Cosmic rays at the energy frontiers. Sci Am 276:44

Crooker NU, Cliver EW, Tsurutani BT (1992) The semi-annual variation of great geomagnetic storms and the post shock Russell-McPherron effect preceding coronal mass ejecta. Geophys Res Lett 19:429

Crooker NU, Gosling JT, Bothmer V, Forsyth RJ, Gazis PR, Hewish A, Horbury TS, Intriligator DS, Jokipii JR, Kota J, Lazarus AJ, Lee MAI, Lucek E, Marsch E, Posner A, Richardson IG, Roelof EC, Schmidt JM, Siscoe GL, Tsurutani BT, Wimmer-Schweingruber RF (1999) CIR morphology, turbulence, discontinuities, and energetic particles: report of working group 2. Space Sci Rev 89:179-220

Crosby NB (2007) Major radiation environments in the heliosphere and their implications for interplanetary travel. In: Space weather-physics and effects, pp 131-171

Crosby NB, Rycroft MJ, Tulunay Y (2006) Overview of a graduate course delivered in Turkey, emphasizing solar-terrestrial physics and space weather. Surv Geophys 27:319-364

Daglis IA (1997a) The role of magnetosphere-ionosphere coupling in magnetic storm dynamics. In: Tsurutani BT, Gonzelez WD, Kamide Y, Arballo JK (eds) Geophysical Monograph Series, vol 98. AGU, Washington

Daglis IA (1997b) Terrestrial agents in the realm of space storms: Missions study oxygen ions. Eos Trans AGU 24:245

Daglis IA (ed) (2001) Space storms and space weather hazards. Kluwer, Dordrecht, p 482

Daglis IA, Kamide Y (2003) The role of magnetosphere-ionosphere coupling in magnetic storm dynamics. In: Sharma AS, Kamide Y, Lakhina GS (eds) Disturbances in geospace: the storm-substorm relationship, vol. 142 of Geophysical Monograph, pp 119-129. American Geophysical Union, Washington

Daglis IA, Thorne RM, Baumjohann W, Orisini S (1999) The terrestrial ring current: origin, formation and decay. Rev Geophys 37:407-438

Daly E, Lemaire J, Heynderickx D, Rodgers D (1996) Problems with models of the radiation belts. IEEE Trans Nucl Sci NS-43:403

Darrouzet F, Gallagher DL, André N, Carpenter DL, Dandouras I, Décréau PME, Keyser JD, Denton RE, Foster JC, Goldstein J (2009) Plasmaspheric density structures and dynamics: properties observed by the CLUSTER and IMAGE missions. Space Sci Rev 145:55-106

Davies JA, Harrison RA, Rouillard AP, Sheeley NR Jr, Perry CH, Bewsher D, Davis CJ, Eyles CJ, Crothers SR, Brown DS (2009) The stereo mission. Geophys Res Lett 36:L02102. doi:10.1029/2008GL036182

Davis CJ, Davies JA, Lockwood M, Rouillard AP, Eyles CJ, Harrison RA (2009) Stereoscopic imaging of an Earth-impacting solar coronal mass ejection: a major milestone for the STEREO mission. Geophys Res Lett 36:L08102. doi:10.1029/2009GL038021

Demirkol M, Inan US, Bell T, Kanekal S, Wilkinson D (1999) Ionospheric effects of relativistic electron enhancement events. Geophys Res Lett 26:3557

Denton MH, Borovsky JE, Skoug RM, Thomsen MF, Lavraud B, Henderson MG, McPherron RL, Zhang JC, Liemohn MW (2006) Geomagnetic storms driven by ICME and CIR-dominated solar wind. J Geophys Res 111:A07S07. doi:1029/2005JA011436

Denton MH, Ulich T, Turunen E (2009) Modification of midlatitude ionospheric parameters in the F2 layer by persistent high-speed solar wind streams. Space Weather 7:S04006. doi:10.1029/2008SW000443

Desorgher L, Flückiger EO, Gurtner M, Moser MR, Bütikofer R (2005) Atmocosmics: a GEANT4 code for computing the interaction of cosmic rays with the Earth's atmosphere. Int J Mod Phys A 20: 6802-6804. doi:10.1142/S0217751X05030132

Desorgher L, Kudela K, Flückiger E, Bütikofer R, Storini M, Kialegee V (2009) Comparison of Earth's megnetospheric magnetic field models in the context of cosmic ray physics. Acta Geophys 57:75-87

DeVore CR, Antiochos SK (2008) Homologous confined filament eruptions via magnetic breakout. Astrophys J 680:740-756 
Dorman LI, Belov AV, Eroshenko EA, Gromova LI, Iucci N, Levitin AE, Parisi M, Ptitsyna NG, Pustilnik LA, Tyasto MI, Vernova ES, Villoresi G, Yanke VG, Zukerman IG (2005) Different space weather effects in anomalies of the high and low orbital satellites. Adv Space Res 36:2530-2536

Dubinin E, Skalsky A, Song P, Savin S et al (2002) Polar-Interball co-ordinated observations of plasma and magnetic field characteristics in the regions of the northern and southern distant cusps. J Geophys Res 107:1053. doi:10.1029/2001JA900068

Dungey JW (1961) Interplanetary magnetic field and the auroral zones. Phys Rev Lett 6:47-48

Dungey JW (1981) Magnetospheric plasmas. Phil Trans R Lond 300:489-496

Ebihara Y, Ejiri M (2003) Numerical simulation of the ring current: review. Space Sci Rev 105:377-452

Echer W, Gonzalez WD, Alves MV (2006) On the geomagnetic effects of solar wind interplanetary magnetic structures. Space Weather 4:S06001. doi:10.1029/2005SW000200

Echer E, Gonzalez WD, Tsurutani BT (2008) Interplanetary conditions leading to superintense geomagnetic storms $\left(D_{\text {st }} \leq-250 \mathrm{nT}\right)$ during solar cycle 23. Geophys Res Lett 35:6. doi:10.1029/2007GL031755

Elkington SR, Hudson MK, Chan AA (1999) Acceleration of relativistic electrons via drift-resonant interaction with toroidal-mode Pc-5 ULF oscillations. Geophys Res Lett 26:3273

Evenson P, Meyer P, Yanagita S, Forrest DJ (1984) Electron-rich particle events and the production of gamma rays by solar flares. Astrophys J 283:439

Eyles CJ, Simnett GM, Cooke MP, Jackson BV, Buffington A, Hick PP, Waltham NR, King JM, Anderson PA, Holladay PE (2003) The solar mass ejection imager (SMEI). Sol Phys 217:319-347. doi: 10.1023/B:SOLA.0000006903.75671.49

Fahr HJ, Neutsch W, Grzedzielski S, Macek W, Ratkiewicz-Landowska R (1986) Plasma transport across the heliopause. Space Sci Rev 43:329-381

Falconer D, Moore R, Gray A (2007) Forecasting solar coronal mass ejections from MDI magnetograms. Am Astron Soc Meet 210:2702

Fan Y, Gibson SE (2007) Onset of coronal mass ejections due to loss of confinement of coronal flux ropes. Astrophys J 668:1232-1245

Farrugia CJ, Burlaga LF, Lepping RP (1997) Magnetic clouds and the quiet-storm effect at Earth. In: Tsurutani BT, Gonzalez WD, Kamide Y, Arballo JK (eds) Magnetic storms, vol 98 of Geophysical Monograph. American Geophysical Union, Washington, pp 91-106

Feldman WC, Symbalisty EMD, Roussel-Dupré RA (1996) Hard X ray survey of energetic electrons from low-Earth orbit. J Geophys Res 101:5195

Feng HQ, Wu DJ, Chao JK (2006) Identification of configuration and boundaries of interplanetary magnetic clouds. J Geophys Res 111:A07S90. doi:10.1029/2005JA011509

Filjar R (2008) A study of direct severe space weather effects on GPS ionospheric delay. J Navig 61:115-128 (Cambridge University Press)

Flury W, Contant JM (2001) The updated IAA position paper on orbital debris. In: Huguette S-L (ed) Proceedings of the 3rd European conference on space debris, 19-21 March 2001, Darmstadt, Germany, ESA SP-473, vol 2, pp 841-849

Fok MC, Moore TE, Kozyra JU, Ho GC, Hamilton DC (1995) Three-dimensional ring current decay model. J Geophys Res 100:9619-9696

Forbes TG, Linker JA, Chen J, Cid C et al (2006) CME theory and models. Space Sci Rev 123:251

Forsyth RJ, Bothmer V, Cid C, Crooker NU et al (2006) ICMEs in the inner heliosphere: origin, evolution and propagation effects. Space Sci Rev 123:383-416

Friedel RHW, Reeves GD, Obara T (2002) Relativistic electron dynamics in the inner magnetosphere-a review. J Atmos Solar Terr Phys 64:265

Fritz TA, Fung SF (eds) (2005) The magnetospheric cusps: structure and dynamics. Surv Geophys 26:95-133

Fritz TA, Chen J, Siscoe GL (2003a) Energetic ions, large diamagnetic cavities, and Chapman-Ferraro Cusp. J Geophys Res 108:1028-1036

Fritz TA, Alothman M, Bhattacharjya J, Matthews D, Chen J (2003b) Butterfly pitch angle distributions observed by ISEE-1. Planet Space Sci 51:205-219

Fry CD, Sun W, Deehr CS, Dryer M, Smith Z, Akasofu SI, Tokumaru Maud Kojima M (2001) Improvements to the HAF solar wind model for space weather predictions. J Geophys Res 106:20985-21001

Fry CD, Dryer M, Deehr CS, Smith Z, Sun W, Akasofu SI, Smith Z (2003) Forecasting solar wind structure and shock arrival times using an ensemble of models. J Geophys Res 108:1070

Fu SY, Zong QG, Pu ZY, Xiao CJ, Korth A, Daly PW, Reme H (2005) Energetic particles observed in the Cusp region during a storm recovery phase. Surv Geophys 26:241-254. doi:10.1007/s10712-005-1881-5

Funk S (2008) VHE Gamma-ray supernova remnants. Adv Space Res 41(3):464-472

Gabriel AH (1974) A magnetic model of the chromosphere-corona transition region. In: Athay RG (ed) Chromospheric fine structure, IAU symposium, vol 56, pp 295-298. D. Redel Publ. Company, Dordrecht 
Gaisser TK (1990) Cosmic rays and particle physics. Cambridge University Press, Cambridge

Ganushkina NY, Pulkkinen TI, Fritz T (2005) Role of substorm-associated impulsive electric fields in the ring current development during storms. Ann Geophys 23:579-591

Garcia HA (2004) Forecasting methods for occurrence and magnitude of proton storms with solar hard X-rays. Space Weather, 2:S06003, 10. doi:10.1029/2003SW000035

Garrett HB (1981) The charging of spacecraft surfaces. Rev Geophys 19:577

Gary DE, Keller CU (2004) Solar and space weather radiophysics. Springer, Heidelberg

Gary DE, Lanzerotti LJ, Nita GM, Thomson DJ (2005) In: Effects of space weather on technology infrastructure, NATO Sci Series, vol 176. Springer, Amsterdam, p 203. doi:10.1007/1-4020-2754-0_11

George JS, Lave KA, Wiedenbeck ME, Binns WR, Cummings AC, Davis AJ, de Nolfo GA, Hink PL, Israel MH, Leske RA, Mewaldt RA, Scott LM, Stone EC, von Rosenvinge TT, Yanasak NE (2009) Elemental composition and energy spectra of galactic cosmic rays during solar cycle 23 . Astrophys $\mathbf{J}$ 698:1666-1681

Georgoulis MK, Rust DM (2007) Quantitative forecasting of major solar flares. Astrophys J Lett 661:L109

Giacalone J, Jokipii JR, K'ota J (2002) Particle acceleration in solar wind compression regions. Astrophys J 573:845-850

Glauert SA, Horne RB (2005) Calculation of pitch angle and energy diffusion coefficients with the PADIE code. J Geophys Res 110:A04206. doi:10.1029/2004JA010851

Gleisner H, Watermann J (2006a) Concepts of medium-range (1-3 days) geomagnetic forecasting. Adv Space Res 37:1116-1123

Gleisner H, Watermann J (2006b) Solar energetic particle flux enhancement as an indicator of halo coronal mass ejection geoeffectivness. Space Weather 4:S06006. doi:10.1029/2006SW000220

Goldstein H (1983) Solar wind fire. NASA Conf. Pub. CP-2280, 731

Goldstein J (2006) Plasmasphere response: tutorial and review of recent imaging results. Space Sci Rev 124. doi:10.1007/s11214-006-9105-y

Gonzalez WD, Joselyn JA, Kamide Y, Kroehl HW, Rostoker G, Tsurutani BT, Vasyliunas VM (1994) What is geomagnetic storm? J Geophys Res 99:5771-5792

Gonzalez WD, Echer E, Clua-Gonzalez G, Tsurutani BT (2007) Interplanetary origin of intense geomagnetic storms $\left(D_{\mathrm{st}}<-100 \mathrm{nT}\right)$ during solar cycle 23. Geophys Res Lett 34:L06101. doi: $10.1029 / 2006$ GL028879

Goodman JM (2005) In space weather \& telecommunications. Springer, New York, p 1

Goodman JM, Aarons J (1990) Ionospheric effects on modern electronic systems. Proc IEE 78:512-528

Gopalswami N, Lara A, Yashiro S, Kaiser MI, Howard RA (2001) Predicting the 1-AU arrival times of coronal mass ejections. J Geophys Res 106:29207-29218

Gopalswamy N (2004) A global picture of CMEs in the inner heliosphere. In: Poletto G, Suess ST (eds) The sun and the heliosphere as an integrated system, vol 317 of Astrophysics and Space Science Library. Kluwer, Dordrecht, pp 201-251

Gopalswamy N (2009) The sun and earth's space environment. In: Proceeding of the 2009 international conference on space science and communication 26-27 Oct, Port Dickson, Negeri Sembilan, Malaysia, pp 5-10

Gopalswamy N, Kaiser ML, Lepping RP, Kahler SW, Ogilvie K, Berdichevesky D, Kondo T, Isobe T, Akioka M (1998) Origin of coronal and interplanetary shocks-a new look with WIND spacecraft data. J Geophys Res 103:307-316

Gopalswamy N, Yashiro S, Krucker S, Stenborg G, Howard RA (2004) Intensity variation of large solar energetic particle events associated with coronal mass ejections. J Geophys Res 109:A12105. doi: 10.1029/2004JA10602

Gopalswamy N, Yashiro S, Akiyama S (2007) Geoeffectiveness of halo coronal mass ejections. J Geophys Res 112:A06112. doi:10.1029/2006JA012149

Gosling JT (1993) The solar flare myth. J Geophys Res 98:18937-18949. doi:10.1029/93JA01896

Gosling JT, Hildner E, MacQueen RM, Munro RH, Poland AI, Ross CL (1974) Mass ejections from the sun-a view from SKYLAB. J Geophys Res 79:4581-4587

Gosling JT, Hundhausen AJ, Bame SJ (1976) Solar wind stream evolution at large heliocentric distancesexperimental demonstration and the test of a model. J Geophys Res 81:2111-2122

Gosling JT, Thomsen MF, Bame SJ, Zwickl RD (1987) The eastward deflection of fast coronal mass ejecta in interplanetary space. J Geophys Res 92:12399-12406. doi:10.1029/JA092iA11p12399

Gosling JT, Bame SJ, Feldman WC, McComas DJ, Phillips JL, Goldstein BE (1993) Counterstreaming suprathermal electron events upstream of corotating shocks in the solar wind beyond approximately 2 AU: ULYSSES. Geophys Res Lett 20:2335-2338 
Gosling JT, Skoug RM, Haggerty DK, McComas DJ (2005) Absence of energetic particle effects associated with magnetic reconnection exhausts in the solar wind. Geophys Res Lett 32:L14113. doi: 10.1029/2005GL023357

Green LM, Matthews SA, Van Driel-Gesztelyi L, Harra LK, Culhane JL (2002) Multi-wavelength observations of an X-class flare without a coronal mass ejection. Solar Phys 205:325-339

Gulyaeva TL, Stanislawaka I (2010) Magnetosphere-associated storms and the autonomous storms in the ionosphere-plasmasphere environment. J Solar Terr Phys 72:90-96

Häder DP, Dachev T (2003) Measurements of solar and cosmic radiation during spaceflight. Surv Geophys 24:229-246

Haggerty DK, Roelof EC (2002) Impulsive near-relativistic solar electron events: delayed injection with respect to solar electromagnetic emission. Astrophys J 579:841-853

Hakamada K, Akasofu SI (1982) Simulation of three dimensional solar wind disturbances and resultion geomagnetic storms. Space Sci Rev 31:3-70

Halley E (1692) An account of the cause of the change of the variation of the magnetical needle with an hypothesis of the structure of the internal parts of the Earth. Phil Trans, xvi:563-587. The paper was read to the Royal Society on 25 November 1691

Hanslmeier A (2003) Space weather-effects of radiation on manned space missions. Hvar Obs Bull 27:159-170

Hastings D, Garrett H (1996) Spacecraft-environment interactions. Cambridge University Press, Cambridge, p 292

Hathaway DH, Wilson RM (2004) What the sunspot record tells us about space climate. Sol Phys 224:5-19

Henderson MG, Reeves GD, Skoug RM, Thomsen MF, Denton MH, Mende SB, Immel TJ, Brandt PC, Singer HJ (2006) Magnetospheric and auroral activity during the April 18, 2002 sawtooth event. J Geophys Res 111:A01303. doi:10.1029/2005JA011320

Hey J (1946) Solar radiations in the 4-6 meter radio wavelength band. Nature 158:234-236

Hildner E (1977) Mass ejections from the solar corona in to interplanetary space. In: Shea MA, Smart DF, Wu ST (eds) Study of travelling interplanetary phenomena 1977. Proceedings of the L.D. de Feiter memorial symposium held in Tel Aviv, Israel, 7-10 June, vol 71 of Astrophysics and Space Science Library. Reidel, Dordrecht, pp 3-20

Holman GD (2003) The effects of low- and high-energy cutoffs on solar flare microwave and hard x-ray spectra. Astrophys J 586:606. doi:10.1086/367554

Hones EW Jr (1979) Plasma flow in the magnetotail and its implications for substorm theories. In: Akasofu SI (ed) Dynamics of the magnetosphere. Proceedings of the A.G.U. Chapman conference 'magnetospheric substorms and related plasma processes', held at Los Alamos Scientific Laboratory, Los Alamos, 9-13, Oct 1978, vol 78 of Astrophysics and Space Science Library, p 545

Horne RB (2002) The contribution of wave particle interactions to electron loss and acceleration in the Earth's radiation belts during geomagnetic storms. Review of Radio Science 1999-2002. Wiley, New York, pp 801-828

Horne RB (2007) Plasma astrophysics: acceleration of killer electrons. Nature Phys 3:590

Horne RB, Thorne RM (1998) Potential waves for relativistic electron scattering and stochastic acceleration during magnetic storms. Geophys Res Lett 25:3011-3014

Horne RB, Meredith NP, Thorne RM, Heynderickx D, Iles RHA, Anderson RR (2003a) Evolution of energetic electron pitches angle distributions during storm time electron acceleration to megaelectronvolt energies. J Geophys Res 108:1016. doi:10.1029/2002JA0099165

Horne RB, Glauert SA, Thorne RM (2003b) Resonant diffusion of radiation belt electrons by whistler-mode chorus. Geophys Res Lett 30:1493. doi:10.1029/2003GL016963

Horne RB, Thorne RM, Shprits YY, Meredith NP, Glauert SA, Smith AJ, Kanekal SG, Baker DN, Engebretson MJ, Posch JL, Spasojevic M, Inan US, Pickett JS, Decreau PME (2005) Wave acceleration of electrons in the Van Allen radiation belts. Nature 437:227-230. doi:10.1038/nature03939

Horne RB, Meredith NP, Glauert SA, Varotsou A, Boscher D, Thorne RM, Shprits YY, Anderson RR (2006) Recurrent magnetic storms: corotating solar wind streams. In: Tsurutani BT, McPherron RL, Gonzalez WD, Lu G, Sobral JHA, Gopalswamy N (eds) Geophys Monogr Series, vol 167. AGU, Washington, p 151

Hovestadt D, Klecker B, Scholer M, Gloeckler G, Ipavich FM, Fan CY, Fisk LA, Ogallagher JJ (1978) Evidence for solar wind origin of energetic heavy ions in the Earth's radiation belt. Geophys Res Lett 5:1055-1057

Howard RA, Michels DJ, Sheeley NR Jr, Koomen MJ (1982) The observation of a coronal transient directed at earth. Astrophys J 263:L101-L104

Howard RA, Sheeley NR Jr, Michels DJ, Koomen MJ (1985) Coronal mass ejections-1979-1981. J Geophys Res 90:8173-8191 
Howard RA et al (2008) Sun Earth connection coronal and heliospheric investigation (SECCHI). Space Sci Rev 136:67-115

Hudson MK, Kotelnikov AD, Li X, Roth I, Temerin M, Wygant J, Blake JB, Gussenhoven MS (1995) Simulation of proton radiation belt formation during the March 24, 1991 SSC. Geophys Res Lett 22:291

Hundhausen AJ (1972) Coronal expansion and solar wind, vol 5 of Physics and Chemistry in Space. Springer, Berlin

Hundhausen AJ (1997) Coronal mass ejections. In: Jokipii JR, Sonett CP, Giampapa MS (eds) Cosmic winds and the heliosphere. University of Arizona Press, Tucson, pp 259-296

Hunsucker RD, Hargreaves JK (2003) The high-latitude ionosphere and its effects on radio propagation. Cambridge University Press, Cambridge, p 617

Huttunen KEJ, Koskinen HEJ (2004) Importance of post-shock streams and sheath region as drivers of intense magnetospheric storms and high-latitude activity. Ann Geophys 22:1729-1738

Huttunen KEJ, Kilpua SP, Pulkkinen A, Viljanen A, Tanskanen E (2008) Solar wind drivers of large geomagnetically induced currents during the solar cycle 23. Space Weather 6:S10002. doi: 10.1029/2007SW000374

Iles RHA, Meredith NP, Fazakerley AN, Horne RB (2006) Phase space density analysis of the outer radiation belt energetic electron dynamics. J Geophys Res 111:A03204. doi:10.1029/2005JA011206

Isliker H, Benz AO (1994) Catalogue of 1-3 GHz solar flare radio emission. Astron Astrophys Suppl Ser 104:145

Iucci N, Parisi M, Storini M, Villoresi G (1979) Forbush decreases: origin and development in the interplanetary space. Nuovo Cimento 2C:1-52

Jackson BV, Buffington A, Hick PP et al (2004) The solar mass ejection imager (SMEI): the mission. Sol Phys 225:177-207. doi:10.007/s11207-004-2766-3

Jacobs C, Poedts S, Van der Holst B (2006) The effect of the solar wind on CME triggering by magnetic foot point shearing. Astron Astrophys 450:793-803

Jensen JM, Lundstedt H, Thompson MJ, Pijpers FP, Rajaguru SP (2004) Application of local-area helioseismic methods as predicters of space weather. In: Danesy D (ed) Helio- and asteroseismology: towards a golden future. Proceedings of SOHO 14/GONG + 2004 Meeting, ESA SP-559, pp 497-500

Jones JBL, Bentley RD, Hunter R, Iles RHA, Taylor GC, Thomas DJ (2001) The practical issues of utilizing a European space weather programme for airline operations. In: Proceedings of ESA space weather workshop, ESA WPP-194

Kahler SW (1992) Solar flares and coronal mass ejections. Annu Rev Astron Astrophys 30:113-141

Kahler SW (2001) Origin and properties of solar energetic particles in space. In: Song P et al (eds) Space weather, vol 125 of Geophysical Monograph. American Geophysical Union, Washington, pp 109-122

Kahler SW, Vourlidas A (2005) Fast coronal mass ejection environments and the production of solar energetic particle events. J Geophys Res 110:A12S01. doi:10.1029/2005JA011073

Kaiser ML, Kucera TA, Davila JM, St. Cyr OC, Guhathakurta M, Christian E (2008) The STEREO mission: an introduction. Space Sci Rev 136:5-16

Kamide Y, Yakoyama N, Gonzalez WD, Tsuruthani BT, Brekke A, Masuda S (1998) Two step development of geomagnetic storms. J Geophys Res 103:6917

Kennewell JA (1989) In: Proceedings of international conference on antennas and propagation (ICAP 89), Part 2 (A90-27776 11-32). NJ, Institution of Electrical Engineers, London, p 334

Kim RS, Cho KS, Moon YJ, Kim YH, Yi Y, Dryer M, Bong SC, Park YD (2005) Forecast evaluation of the coronal mass ejection (CME) geoeffectiveness using halo CMEs from 1997 to 2003. J Geophys Res 110:A11104. doi:10.1029/2005JA011218

Kintner PM, Coster AJ, Fuller-Rowell T, Mannucci AJ, Mendillo M, Heelis R (eds) (2008) Midlatitude ionospheric dynamics and disturbances. American Geophysical Union, Washington, p 327

Kivelson MG, Russell CT (eds) (1995) Introduction to space physics. Cambridge University Press, Cambridge, p 568

Klassen A, Bothmer V, Mann G, Reiner MJ, Krucker S, Vourlidas A, Kunow H (2002) Solar energetic electron events and coronal shocks. Astron Astrophys 385:1078

Klassen A, Krucker S, Kunow H, Müller-Mellin R, Wimmer-Schweingruber R, Mann G, Posner A (2005) Solar energetic electrons related to the October 28, 2003 flare. J Geophys Res 110:A09S04. doi: 10.1029/2004JA010985

Klecker B (2009) Energetic particles in the heliosphere, invited talk. In: Proceedings of 21st ECRS, Kosice, 2008, pp 27-38

Klinkrad H (2007) On-orbit risk reduction—collision avoidance. J Aerosp Eng 221:955-962

Klobuchar JA, Kunches JM, VanDierendonck AJ (1999) Eye on the ionosphere: potential solar radio burst effects on GPS Signal to noise. GPS Solut 3(2):69-71 
Klos Z, Rothkaehl H, Zbyszyski Z, Kuznetsov S, Gregorian O, Budko NI, Prutensky IS, Pulinets SA (1997) In: Sadowski M, Rothkaehl H (eds) Plasma 97: research and applications of plasmas, p 1

Klotz I (2010) A new era of space weather forecasting. Space Weather 8:S05003. doi:10.1029/2010SW 000587

Koons HC (1980) Characteristics of electrical discharges on the P78-2 satellite (SCATHA). In: 18th Aerospace sciences meeting, AIAA 80-0334, Pasadena

Koons HC, Mazur JE, Selesnick RS, Blake JB, Fennel JF, Roeder JL, Anderson PC (1999) The impact of the space environment on space systems, Engineering and Technology Group, The Aerospace Corp., Report TR-99(1670), El Segundo

Koskinen H, Tanskanenl E, Pirjola R, Pulkkinen A, Dyer C, Rodgers D, Cannon P, Mandeville JC, Boscher D (2001) Space weather effects (catalogue: http://www.esa-spaceweather.net/spweather)

Krucker S, Oakley PH, Lin RP (2009) Spectra of solar impulsive electron events observed near Earth. Astrophys J 691:806-810

Kudela K (2009) On energetic particles in space. Acta Phys Slovaca 59:537-652

Kudela K, Brenkus R (2004) Cosmic ray decreases and geomagnetic activity: list of évents 1982-2002. J Atmos Solar Terr Phys 66:1121-1126

Kudela K, Storini M, Hofer MY, Belov A (2000) Cosmic Rays in Relation to Space Weather. Space Sci Rev 93:153-174

Kudela K, Bǔc'1k R, Bob'1k P (2008) On transmissivity of low energy cosmic rays in disturbed magnetosphere. Adv Space Res 42:1300-1306

Kuznetsov SN, Kurt VG, Yushkov BY, Myagkova IN, Kudela K, Kǎšsovicov'a J, Slivka M (2005) Proton acceleration during 20 January 2005 solar flare: CORONAS-F observations of high-energy gamma emission and GLE. In: Proceedings of 29th ICRC, Pune, vol 1, pp 49-52

Kuznetsov SN, Myagkova IN, Yushkov BY, Denisov YI, Muraveva EA, Kudela K (2007) Dynamics of the earth radiation belts during strong magnetic storms based on CORONAS-F data. Solar Syst Res 41:338-347

Laitinen TV, Janhunen P, Pulkkinen TI, Palmroth M, Koskinen HEJ (2006) On the characterization of magnetic reconnection in MHD simulations. Ann Geophys 24:3059-3069

Lakhina GS, Tsurutani BT (1998) Explosive energy release by disruption of current sheets. Phys Scr 67. doi: 10.1088/0031-8949/1998/T74/013

Lanzerotti LJ (1979) Impacts of ionospheric/magnetospheric processes on terrestrial science and technology. In: Solar system plasma physics, vol III. Noth-Holland Publishing Co., p 319

Lanzerotti LJ (2001) Space weather effects on communications. In: Daglis IA (ed) Space storms and space weather hazards, NATO Science series. Kluwer, Dordrecht

Lanzerotti LJ (2007) Space weather effects on communications. In: Bothmer V, Daglis IA (eds) Space weather-physics and effects. Springer, Praxis Publishing, Chichester, pp 247-268

Lanzerotti L, Medford L, Maclennan C, Thomson D (1995) Studies of large-scale earth potentials across oceanic distances. AT \& T Tech J (May/June):73

Lanzerotti L, Thomson D, Maclennan C (1999) Engineering issues in space weather, Modern Radio Science. Published for the International Union of Radio Science by Oxford University Press

Lanzerotti LJ, Gary DE, Nita GM, Thomson DJ, McIennan CG (2005) Noise in wireless systems from solar radio bursts. Adv Space Res 36:2253

Laurenza M, Cliver EW, Hewitt J, Storini M, Ling AG, Balch CC, Kaiser ML (2009) A technique for shortterm warning of solar energetic particle events based on flare location, flare size, and evidence of particle escape. Space Weather 7. doi:10.1029/2007SW000379

Lavraud B, Reme H, Dunlop MW, Bosqued JM, Dandouras I, Sauvaud JA, Keling A, Phanr TD, Lundin TD, Cargil PJ, Escoubet CP, Carlson CW, Mcfadden JP, Parks GK, Moebius E, Kistler LM, Amata E, Bavassano-Cattaneo MB, Korth A, Klecker B, Balogh A (2005) Cluster observes the high-altitude cusp region. Surv Geophys 26:135-175

Lazutin LL, Logachev YI (2009) Contribution of solar cosmic rays to the formation of the Earth's proton radiation belt. Cosmic Res 47:374-377

Lean J (1989) Contribution of ultraviolet irradiance variations to changes in the sun's total irradiance science. Science 244:197. doi:10.1126/science.244.4901.197

Lean J (1991) Variation in the sun's radiative output. Rev Geophys 29:505-535

Lee MA (2005) Coupled hydromagnetic wave excitation and ion acceleration at an evolving coronal/ interplanetary shock. Astrophys J Suppl 158:38-67

Lemaignen L (1988) Study of biological effects and radiation protection to future European manned space flights. ESA contract 6988/86/NL/PP (SC), AMD-BA Report, 323

Lemaire JF, Gringauz KI (1998) The earth's plasmasphere. Cambridge University Press, Cambridge, p 350 
Li Y, Luhmann J (2006) Coronal magnetic field topology over filament channels: implication for coronal mass ejection initiations. Astrophys J 648:732-740

Li X, Temerin MA (2001) The electron radiation belt. Space Sci Rev 95:569-580

Li Y, Luhmann JG, Hoeksema JT, Zhao XP, Arge CN (2001) Visualizing CMEs and predicting geomagnetic storms from solar magnetic fields. In: Song P, Singer HJ, Siscoe G (eds) Space weather, Geophysics Monograph Series, vol 125. AGU, Washington, pp 177-182

Lilensten J (ed) (2007) Space weather: research towards applications in Europe, Astrophysics and Space Science Library, vol 344. Springer, Dordrecht, p 330

Lilensten J, Belehaki A (2009) Developing the scientific basis for monitoring, modelling and predicting space weather. Acta Geophys 57:1-14

Lin RP (1980) Energetic particles in space. Solar Phys 67:393-399

Lin RP (1985) Energetic solar electrons in the interplanetary medium. Solar Phys 100:537-561

Lin RP, Mewaldt RA, Van Hollebeke MAI (1982) The energy spectrum of $20 \mathrm{keV}-20 \mathrm{MeV}$ electrons accelerated in large solar flares. Astrophys J 253:949-962

Lindsay GM, Rusell CT, Luhmann (1995) Coronal mass ejection and stream interaction region characteristics and their potential geomagnetic effectiveness. J Geophys Res 100:16999

Liu CM, Liu LG, Pirjola R, Wang ZZ (2009) Calculation of geomagnetically induced currents in mid to low-latitude power grids based on the plane wave method: a preliminary case study. Space Weather 7:S04005. doi:10.1029/2008SW000439

Lockwood JA (1971) Forbush decreases in the cosmic radiation. Space Sci Rev 12:658-715

Looper MD, Blake JB, Mewaldt RA (2005) Response of the inner radiation belt to the violent Sun-Earth connection events of October-November 2003. Geophys Res Lett 32:L03S06. doi:10.1029/2004GL 021502

Lopez RE, Baker DN, Allenet JE (2004) Sun unleashes Hallowe'en storm. Eos Trans AGU 85:105-108

Lorentzen KR, Mazur JE, Looper MD, Fennell JF, Blake JB (2002) Multisatellite observations of MeV ion injections during storms. J Geophys Res 107:1231. doi:10.1029/2001JA000276

Low BC (2001) Coronal mass ejections, magnetic flux ropes, and solar magnetism. J Geophys Res 106 : 25141-25164

Lynch BJ, Antiochos SK, DeVore CR, Luhmann JG, Zurbuchen TH (2008) Topological evolution of a fast magnetic breakout CME in three dimensions. Astrophys J 683:1192

Mann G (2005) Monitoring of the solar activity by LOFAR. Astron Nachr 326:618

Manoharan PK et al (2004) Influence of coronal mass ejection interaction on propagation of interplanetary shock. J Geophys Res 109:A06109. doi:10.1029/2003JA010300

Marusek JA (2007) Solar storm threat analysis. Impact 1-29. URL: http://www.breadandbutterscience.com/ SSTA

Mathie RA, Mann IR (2000) A correlation between extended intervals of ULF wave power and storm-time geosynchronous relativistic electron flux enhancements. Geophys Res Lett 27:3261

McAllister AH, Crooker NU (1997) Coronal mass ejections, corotating interaction regions, and geomagnetic storms. In: Crooker N, Joselyn JA, Feynman J (eds) Coronal mass ejections, Geophysics Monograph Series, vol 99. AGU, Washington, pp 279-289

McComas DJ, Gosling JT, Winterhalter D, Smith EJ (1988) Interplanetary magnetic field draping about fast coronal mass ejecta in the outer heliosphere. J Geophys Res 93:2519-2526

McComas DJ, Elliott HA, Schwadron NA, Gosling JT, Skoug V, Goldstein BE (2003) The three-dimensional solar wind around solar maximum. Geophys Res Lett 30:1-24. doi:10.1029/2003GL017136

McCracken KG, Smart DF, Shea MA, Dreschhoff GAM (2001) 400 years of large fluence solar proton events. Conf Pap Int Cosmic Ray Conf 8:3209-3212

McCracken KG, McDonald FB, Beer J, Raisbeck G, Yiou F (2004) A phenomenological study of the longterm cosmic ray modulation, 850-1958 AD. J Geophys Res 109:A12103. doi:10.1029/2004JA010685

McKenna-Lawlor SMP (2008) Predicted interplanetary shocks/particles at Mars compared with in-situ observations: an overview. Planet Space Sci 56:1703-1712

McPherron RL (1970) Growth phase of magnetospheric substorms. J Geophys Res 75:5592

McPherron RL (1997) The role of substorms in generation of magnetic storms. In: Tsurutani BT, Gonzalez WD, Kamide Y, Arballo JK (eds) Magnetic storms, Geophysical Monograph Series, vol 98. American Geophysical Union, Washington, p 131

McPherron RL, Russell CT, Aubry MP (1973) Satellite studies of magnetospheric substorms on August 15, 1968. J Geophys Res 78:3131

Melrose DB (1982) Plasma Astrophysics. Gordon \& Breach, London

Meredith NP, Horne RB, Anderson RR (2001) Substorm dependence of chorus amplitudes: implications for the acceleration of electrons to relativistic energies. J Geophys Res 106:13165-13178 
Meredith NP, Horne RB, Thorne RM, Anderson RR (2003) Favored regions for chorus-driven electron acceleration to relativistic energies in the Earth's outer radiation belt. Geophys Res Lett 30:1871. doi: 10.1029/2003GL017698

Meredith NP, Horne RB, Clilverd MA, Horsfall D, Thorne RM, Anderson RR (2006) Origin of plasmaspheric hiss. J Geophys Res 111:A09217. doi:10.1029/2006JA011707

Messerotti M (2008) Observing, modelling and predicting the effects of solar radio bursts on radio communications. In: Exploring the solar system and the universe. AIP Conference Proceedings, vol 1043, pp 277-283

Messerotti M, Zuccarello F, Guglielmino SI, Bothmer V, Lilensten J, Noci G, Storini M, Lundstedt H (2009) Solar weather event modelling and prediction. Space Sci Rev. doi:10.1007/s11214-009-9574-X

Messerschmidt W (1933) Über Schwankungsmessungen der Ultrastrahlung II. Zeitschrift für Physik 85:332-335

Mewaldt RA, Cohen CMS, Labrador AW, Leske RA, Mason GM, Desai MI, Looper MD, Mazur JE, Selesnick RS, Haggerty DK (2005) Proton, helium, and electron spectra during the large solar particle events of October-November 2003. J Geophys Res 110:A09S18. doi:10.1029/2005JA011038

Michałek G, Gopalswamy N, Yashiro S (2003) A new method for estimating widths, velocities, and source location of halo coronal mass ejections. Astrophys J 584:472-478

Miki'c Z, Linker J (1994) Disruption of coronal magnetic field arcades. Astrophys J 430:898-912

Miroshnichenko LI (2008) Solar cosmic rays in the system of solar-terrestrial relations. J Atmos Solar Terr Phys 70:450-466

Miyoshi Y, Morioka A, Misawa H (2000) Long term modulation of low altitude proton radiation belt by the earth's atmosphere. Geophys Res Lett 27:2169

Mizera PF (1983) A summary of spacecraft charging results. J Spacecraft Rockets 20:438

Molinski TS (2002) Why utilities respect geomagnetically induced currents. J Atmos Solar Terr Phys 64:1765-1778

Moon YJ, Cho KS, Dryer M, Kim YH, Bong SC, Chae J, Park YD (2005) New geoeffective parameters of very fast halo coronal mass ejections. Astrophys J 624:414-419

Moorer DR, Baker DN (2001) Specification of energetic magnetospheric electrons. In: Song P, Singer HJ, Siscoe GL (eds) Space weather, vol 125, Geophysical Monograph. American Geophysical Union, Washington, pp 3321-3328

Moses D, Droge W, Meyer P, Evenson P (1989) Characteristics of energetic solar flare electron spectra. Astrophys J 346:523-530

Mulligan T, Russell CT (2001) Multi-spacecraft modeling of the flux rope structure of interplanetary coronal mass ejections: cylindrically symmetric versus non-symmetric topologies. J Geophys Res 106: 10581-10596

Ng CK, Reames DV (2008) Shock acceleration of solar energetic protons: the first 10 minutes. Astrophys J Lett 686:L123-L126

Noël S (1997) Decay of the magnetospheric ring current: a Monte Carlo simulation. J Geophys Res 102:2301-2308

Noyes RW, Withbore GL (1972) The solar EUV emitting plasma. Space Sci Rev 13:612-637

O'Brien K (2005) The theory of cosmic-ray and high-energy solar-particle transport in the atmosphere. In: Proceedings of the 7th international symposium on the natural radiation environment, pp 29-44

O'Sullivan D (2007) Exposure to galactic cosmic radiation and solar energetic particles. Radiat Prot Dosim 125:407-411

Ondoh T, Marubashi K (eds) (2001) Science of space environment. Ohmsha IOS Press, Japan, p 302

Osherovich V, Burlaga LF (1997) Magnetic clouds. In: Crooker N, Joselyn JA, Feynman J (eds) Coronal mass ejections, vol 99, Geophysical Monograph. American Geophysical Union, Washington, pp 157-168

Parker EN (1957) Newtonian development of the dynamical properties of ionized gases of low density. Phys Rev 107:924

Paschmann G (1997) Observational evidence for transfer of plasma across the magnetopause. Space Sci Rev 80:217-234

Pick M, Klein KL, Trottet G (1990) Meter-Decimeter and Microwave Radio Observations of Solar Flares. Astrophys J Supl 73:165

Pirjola R (1998) Modeling the electric and magnetic field at the Earth surface due to an auroral electrijet. J Atmos Solar Terr Phys 60:1139-1148

Pirjola R (2007) Space weather effects on power grids. In: Bothmer V, Daglis L (eds) Space weather: physics, effects. Springer, Berlin, pp 269-288

Pirjola R, ViIjanen A, Amm O, Pulkkinen A (1999) Power and pipelines (ground systems). In: Proceedings of a workshop on space weather, Nov 1998, ESA WPP-155 
Pokhotelov D, Lotko W, Streltsov AV (2004) Simulations of resonant Alfvén waves generated by artificial HF heating of the auroral ionosphere. Ann Geophys 22:2943

Priest ER, Forbes TG (2000) Magnetic reconnection: MHD theory and applications. Cambridge University Press, Cambridge

Priest ER, Forbes TG (2002) The magnetic nature of solar flares. Astron Astrophys Rev 10:313-377. doi: $10.1007 / \mathrm{S} 001590100013$

Priest ER, Parnell CE, Martin SF (1994) A converging flux model of an X-ray bright point and an associated canceling magnetic feature. Astrophys J 427:459-474

Prolss GW (2004) Physics of the Earth's space environment. Springer, New York, p 514

Pulkkinen T (2007) Space weather: terrestrial perspective. Living Rev Solar Phys 4:1. http://www.ava.fmi.fi

Pulkkinen TI, Baker DN, Pellinen RJ, Bückner J, Koskinen HEJ, Lopez RE, Dyson RL, Frank LA (1992) Particle scattering and current sheet stability in the geomagnetic tail during the substorm growth phase. J Geophys Res 97:19283

Pulkkinen TI, Ganushkina NY, Tanskanen EI, Kubyshkina MV, Reeves GD, Thomsen MF, Russell CT, Singer HJ, Slavin JA, Gjerloev JW (2006) Magnetospheric current systems during stormtime sawtooth events. J Geophys Res 111:A07S08. doi:10.1029/2005JA011447

Qahwaji R, Colak T (2007) Automatic short-term solar flare prediction using machine learning and sunspot associations. Solar Phys 241:195-211. doi:10.1007/s11207-006-0272-5

R'egnier S, Priest ER (2007) Nonlinear force-free models for the solar corona: I. Two active regions with very different structure. Astronomy Astrophys 468:701-709

Ramaty R, Murphy RJ, Kozlovsky B, Lingenfelter RE (1983) Gamma ray lines and neutrons from solar flares. Solar Phys 86:395

Ramaty R, Mandzhavidze N, Hua X (eds) (1996) High energy solar physics, Proceedings of the conference held in Greenbelt, Aug 1995, vol 374 of AIP Conference proceedings. American Institute of Physics, Woodbury

Rastogi RG (1999) Geomagnetic storm effects at low latitude. Ann Geophys 17:438

Reames DV (1999) Particle acceleration at the sun and in the heliosphere. Space Sci Rev 90:413-491

Reames DV (2001) SEP: Space weather hazard in interplanetary space. In: Song PS, Singer HJ, Siscoe GL (eds) Space weather, Geophysical Monograph, vol 125. American Geophysical Union, Washington, p 101

Reames DV, Barbier LM, Ng CK (1996) The spatial distribution of particles accelerated by coronal mass ejection-driven shocks. Astrophys J 466:473-486. doi:10.1086/177525

Rees MH (1989) Physics and chemistry of the upper atmosphere. Cambridge University Press, Cambridge, p 289

Reeves GD, Baker DN, Belian RD, Blake JB et al (1998) The global response of relativistic radiation belt electrons to the January 1997 magnetic cloud. Geophys Res Lett 25:3265-3268

Richardson IG, Zwickl RD (1984) Low energy ions in corotating interaction regions at 1 AU: observations. Planet Space Sci. 32:1179-1193

Rodger CJ, Cho M, Clilverd MA, Rycroft MJ (2001) Lower ionospheric modification by lightning-EMP: simulation of the night ionosphere over the United States. Geophys Res Lett 28:199-202

Rodriguez L, Zhukov AN, Cid C, Cerrato Y, Saiz E, Cremades H, Dasso S, Menvielle M, Aran A, Mandrini C, Poedts S, Schmieder B (2009) Three frontside full halo coronal mass ejections with a nontypical geomagnetic response. Space Weather 7:S06003. doi:10.1029/2008SW000453

Root HG (1979) Earth-current effects on communication-cable power subsystems. IEEE Trans Electromagn Compat EMC-21:87

Rothkaehl H, Klos Z (2003) Broadband HF emissions as an indicator of global changes within the ionosphere. In: Plasma processes in the near-earth space: Interball and beyond, vol 31, pp 1371-1376

Rothkaehl H, Parrot M (2005) Electromagnetic emissions detected in the topside ionosphere related to the human activity. J Atmos Solar Terr Phys 67:821-828

Rottman G (1999) Solar ultraviolet irradiance and its temporal variation. J Atmos Solar Terr Phys 61:37-44

Roussev II, Sokolov IV (2006) Models of solar eruptions: recent advances from theory and simulations. In: Solar eruptions and energetic particles, Geophysics Monograph Series, vol 165. American Geophysical Union, Washington, p 89

Russell CT (2008) The STEREO mission. Space Sci Rev 136:1-4

Russell CT, McPherron RL (1973) Semiannual variation of geomagnetic activity. J Geophys Res 78:92

Ryan JM, Lockwood JA, Debrunner H (2000) Solar energetic particles. Space Sci Rev 93:35-53

Rycroft MJ, Odzimek A (2010) Effects of lightning and sprites on the ionospheric potential, and threshold effects on sprite initiation, obtained using an analog model of the global atmospheric electric circuit. J Geophys Res 115:A00E37. doi:10.1029/2009JA014758 
Rycroft MJ, Odzimek A, Arnold NF, Fullekrug M, Kulak A, Neubert T (2007) New model simulations of the global atmospheric electric circuit driven by thunderstorms and electrified shower clouds: the roles of lightning and sprites. J Atmos Solar Terr Phys 69:2485-2509. doi:10.1016/j.jastp.2007.09.004

Sandel BR, King RA, Forrester WT, Gallagher DL, Broadfoot AL, Curtis CC (2001) Initial results from the IMAGE extreme ultraviolet imager. Geophys Res Lett 28:1439

Scherer K, Fichtner H, Heber B, Mall U (eds) (2005) Space weather: the physics behind the slogan. Springer, Dordrecht, p 300

Scholer M (1999) Origin, injection, and acceleration of CIR particles: theory, report of working group. Space Sci Rev 89:369-399

Schrijver CJ (2007) A characteristic magnetic field pattern associated with all major solar flares and its use in flare forecasting. Astrophys J Lett 655:L117. doi:10.1086/511857

Schunk R, Nagy A (2009) Ionospheres: physics, plasma physics and chemistry, 2nd edn. Cambridge University Press, Cambridge, p 628

Schunk RW, Sojka JJ (1996) Ionosphere-thermosphere space weather issues. J Atmos Terr Phys 58: 1527-1574

Schwadron NA, Fisk LA, Gloeckler G (1996) Statistical acceleration of interstellar pick-up ions in corotating interaction regions. Geophys Res Lett 23:2871-2874. doi:10.1029/96GL02833

Schwenn R (2006a) Solar wind sources and their variations over the solar cycle. Space Sci Rev 124:51-76

Schwenn R (2006b) Space weather: the solar perspective. Living Rev Solar Phys 3, lrsp-2006-2. http://www.ava.fmi.fi

Schwenn R, Lago AD, Huttunen E, Gonzalez WD (2005) The association of coronal mass ejections with their effects near the earth. Ann Geophys 23:1033-1059

Sckopke N, Paschmann G, Rosenbauer H, Fairfield DH (1976) Geophys Res Lett 81:2687

Selesnick RS, Looper MD, Mewaldt RA (2007) A theoretical model of the inner proton radiation belt. Space Weather 5:S04003. doi:10.1029/2006SW000275

Sergeev VA, Mitchell DG, Russell CT, Williams DJ (1993) Structure of the tail plasma/current sheet at 11 Re and its changes in the course of a substorm. J Geophys Res 98:227-230

Sergeev VA, Pellinen RJ, Pulkkinen TI (1996a) Steady magnetospheric convection: a review of recent results. Space Sci Rev 75:551-604

Sergeev VA, Pulkkinen TI, Pellinen RJ (1996b) Coupled-mode model for the magnetospheric dynamics. J Geophys Res 101:13047

Shea MA, Smart DF (1998) Space weather: the effects on operation in space. Adv Space Res 22:29

Shea MA, Smart DF (2000a) Fifty years of cosmic radiation data. Space Sci Rev 93:229-262

Shea MA, Smart DF (2000b) Cosmic ray implications for human health. Space Sci.Rev 93:187-205

Sheldon RB, Spence HE, Sullivan JS, Fritz TA, Chen J (1998) The discovery of trapped energetic electrons in the outer cusp. Geophys Res Lett 25:1825-1828

Shelley EG, Johnson RG, Sharp RD (1972) Satellite observations of energetic heavy ions during a geomagnetic storm. J Geophys Res 77:6104-6110

Shih AY, Lin RP, Smith DM (2009) RHESSI observations of the proportional acceleration of relativistic $>0: 3 \mathrm{MeV}$ electrons and $>30 \mathrm{MeV}$ protons in solar flares. Astrophys J Lett 698:L152-L157

Shprits YY, Thorne RM, Horne RB, Glauert SA, Cartwright M, Russell CT, Baker DN, Kanekal SG (2006) Acceleration mechanism responsible for the formation of the new radiation belt during the 2003 Halloween solar storm. Geophys Res Lett 33:L05104. doi:10.1029/2005GL024256

Shprits Y, Kondrashov D, Chen Y, Thorne R, Ghil M, Friedel R, Reeves G (2007) Reanalysis of relativistic radiation belt electron fluxes using CRRES satellite data, a radial diffusion model, and a Kalman filter. J Geophys Res 112:A12216. doi:10.1029/2007JA012579

Siingh D, Singh RP (2010) The role of cosmic rays in the earth's atmospheric processes. Pramana J Phys 74:153-168

Siingh D, Gopalakrishnan V, Singh RP, Kamra AK, Singh S, Pant V, Singh R, Singh AK (2007) The atmospheric global electric circuit: an overview. Atmos Res 84:91-110

Singer SF (1958) Radiation belt and trapped cosmic-ray albedo. Phys Rev Lett 1:171

Singer HJ, Heckman GR, Hirman JW (2001) Space weather forecasting: a grand challenge. In: Song P et al (eds) Space weather, vol 125, Geophysical Monograph. American Geophysical Union, Washington, pp 23-29

Singh AK (2003) Solar wind and space weather. In: Singh RP, Singh R, Singh AK (eds) Solar terrestrial environment: space weather. Allied Publishers, New Delhi, pp 57-68

Singh AK, Singh RP (2003) Space weather-causes, consequences and predictions. Indian J Phys 77B(6): 611-616

Singh DK, Singh RP, Kamra AK (2004) The electrical environment of the earth's atmosphere: a review. Space Sci Rev 113:375-408 
Singh AK, Singh RP, Siingh D (2010) State study of earth's plasmasphere: a review. Planet Space Sci (submitted)

Skone S, Cannon ME (1998) Detailed analysis of auroral zone WADGPS ionosphere grid accuracies during magnetospheric substorm event. In: Proceedings of the ION GPS-98, Nashville

Skone S, Yousuf R (2007) Performance of satellite based navigation for marine users during ionospheric disturbances. Space Weather 5:S01006. doi:10.1029/2006SW000246

Song P, Singer HJ, Siscoe GL (eds) (2001) Space weather. American Geophysical Union, Washington, p 440

Southworth GC (1945) Microwave radiation from the sun. J Franklin Inst 239:285-297

Srivastava N (2005) A logistic regression model for predicting the occurrence of intense geomagnetic storms. Ann Geophys 23:2969-2974

St Cyr OC, Plunkett SP, Michels DJ, Paswaters SE, Koomen MJ, Simnett GM, Thompson BJ, Gurman JB, Schwenn R, Webb DF, Hildner E, Lamy PL (2000) Properties of coronal mass ejections: SOHO LASCO observations from January 1996 to June 1998. J Geophys Res 105:18169-18186

Stone EC, Cohen CMS, Cook WR, Cummings AC, Gauld B, Kecman B, Leske RA, Mewaldt RA, Thayer MR, Dougherty BL, Grumm RL, Milliken BD, Radocinski RG, Wiedenbeck ME, Christian ER, Shuman S, Trexel H, Von Rosenvinge TT, Binns WR, Crary DJ, Dowkontt P, Epstein J, Hink PL, Klarmann J, Lijowski M, Olevitch MA (1998) The cosmic-ray isotope spectrometer for the Advanced Composition Explorer. Space Sci Rev 86:285-356

Sugiara M, Kamei T (1991) Equatorial $D_{\text {st }}$ index 1957-1986. IAGA Bull. No. 40. ISGI Publ. Office, France, $246 \mathrm{pp}$

Summers D, Thorne RM (2003) Relativistic electron pitch-angle scattering by electromagnetic ion cyclotron waves during geomagnetic storms. J Geophys Res 108:1143. doi:10.1029/2002JA009489

Sun W, Deehr CS, Fry CD, Dryer M, Smith Z, Akasofu SI (2008) Simulation of SMEI and STEREO-like views of the solar wind following the solar flares of 27-29 May 2003. Space weather 6:S03006. doi: 10:1029/2006SW000298

Surgiara M (1965) Ann Int Geophys Year 35:9-45

Svestka Z (1976) Solar flares, Geophysics and Astrophysics Monographs Series, vol 8. D. Reidel, Dordrecht

Svestka Z, Simon P (eds) (1976) Catalog of solar particle events 1955-1969, Astrophysics and Space Science Library. Reidel, Dordrecht

Tascione TF (1994) Introduction to the space environment, 2nd edn. Krieger Publishing Company, Florida, p 164

Thernisien AFR, Howard RA, Vourlidas A (2006) Modeling of flux rope coronal mass ejections. Astrophys J 652:763

Tinsley BA, Hodges RR Jr, Rhorbaugh RP (1986) Monte Carlo models for the terrestrial exosphere over a solar cycle. J Geophys Res 91:13631

Todd D (2000, March 6) Letter to Space News, p 12

Tóth G, Sokolov IV, Gombosi TI, Chesney DR, Clauer CR, De Zeeuw DL, Hansen KC, Kane KJ, Manchester WB, Oehmke RC, Powell KG, Ridley AJ, Roussev II, Stout QF, Volberg O (2005) Space weather modeling framework: a new tool for the space science community. J Geophys Res 110. doi: 10.1029/2005JA011126

Trakhtengerts VY, Rycroft MJ (2008) Whistler and Alfven mode cyclotron masers in space. Cambridge University Press, Cambridge, p 354

Tribble AC (1995) The space environment: implications for spacecraft design. Princeton University Press, Princeton, p 204

Trichtchenko L, Boteler DH (2001) Specification of geomagnetically induced electric fields and currents in pipelines. J Geophys Res 106:21039-21048

Tripathi DK, Bothmer V, Cremades H (2004) The basic characteristics of EUV post-eruptive arcades and their role as tracers of coronal mass ejection source regions. Astron Astrophys 422:337

Trivedi NB, Vitorello I et al (2007) Geomagnetically induced currents in an electric power transmission system at low latitudes in Brazil: a case study. Space Weather 5:S04004. doi:10.1029/2006SW000282

Tsubouchi K, Omura Y (2007) Long term occurrence probabilities of intense geomagnetic storm events. Space Weather 5:S12003. doi:10.1029/2007SW000329

Tsurutani BT (2001) The interplanetary causes of magnetic storms, substorms and geomagnetic quiet. In: Daglis IA (ed) Space storms and space weather hazards. Kluwer, Dordrecht, pp 103-130

Tsurutani BT, Gonzalez WD (1987) The cause of high-intensity long-duration continuous AE activity (HILDCAAs): interplanetary Alfvén wave trains. Planet Space Sci 35:40

Tsurutani BT, Smith EJ, Gonzalez WD, Tang F, Akasofu SI (1988) Origin of interplanetary southward magnetic fields responsible for major magnetic storms near solar maximum (1978-1979). J Geophys Res 93:8519-8531 
Tsurutani BT, Gonzalez WD, Gonzalez ALC, Tang F, Arballo JK, Okada M (1995a) Interplanetary origin of geomagnetic activity in the declining phase of the solar cycle. J Geophys Res 100:21717

Tsurutani BT, Ho CM, Arballo JK, Goldstein BE, Balogh A (1995b) Large amplitude IMF fluctuations in corotating interaction regions: Ulysses at mid latitudes. Geophys Res Lett 22:3397

Tsurutani BT, Gonzalez WD, Gonzalez ALC, Guarnieri FL, Gopalswamy N, Grande M, Kamide Y, Kasahara Y, Lu G, Mann I, McPherron R, Soraas F, Vasyliunas V (2006) Corotating solar wind streams and recurrent geomagnetic activity. J Geophys Res 111:A07S01. doi:10.1029/2005JA011273

Tu CY, Zhou C, Marsch E, Xia LD, Zhao L, Wang JX et al (2005) Solar wind origin in coronal funnels? Science 308:519. doi:0.1126/science.1109447

Turner R (ed) (1996) Foundations of solar particle event risk management strategies, in findings of the risk management workshop for solar particle events, publication NASA Grant NAGW-4166. ANSER, Arlington

Tverskaya LV, Pavlov NN, Blake JB, Selesnick RS, Fennell JF (2003) Predicting the L position of the storm-injected relativistic electron belt. Adv Space Res 31:1039-1044

Tyasto MI, Danilova OA, Dorman LI, Dvornikov VM, Sdobnov VE (2008) On the possibility to check the magnetosphere's model by CR: the strong geomagnetic storm in November 2003. Adv Space Res 42:1556-1563

Ugarte-Urra I, Warren HP, Winebarger AR (2007) The magnetic topology of coronal mass ejection sources. Astrophys J 662:1293-1301

Vainio R, Laitinen T (2007) Monte Carlo simulations of coronal diffusive shock acceleration in selfgenerated turbulence. Astrophys J 658:622. doi:10.1086/510284

Vainio R, Laitinen T (2008) Simulations of coronal shock acceleration in self- generated turbulence. J Atmos Solar Terr Phys 70:467

Vainio R, Desorgher L, Heynderickx D, Storini M, Flückiger E, Horne RB, Kovaltsov GA, Kudela K, Laurenza M, McKenna-Lawlor S, Rothkaehl H, Usoskin IG (2009) Dynamics of the earth's particle radiation environment. Space Sci Rev 147:187-231. doi:10.1007/s11214-009-9496-7

Valach F, Hejda P, Bochnı'ček J (2007) Geoeffectiveness of XRA events associated with RSP II and/or RSP IV estimated using the artificial neural network. Stud Geophys Geod 51:551-562

Valach F, Revallo M, Bochnicek J, Hejda P (2009) Solar energetic particle flux enhancement as a predictor of geomagnetic activity in a neural network-based model. Space Weather 7:S04004. doi: $10.1029 / 2008$ SW000421

Vampola A (1987) The aerospace environment at high altitudes and its implications for spacecraft charging and communications. J Electrostat 20:21

Van Allen JA, Frank LA (1959) Radiation around the earth to a radial distance of $107400 \mathrm{~km}$. Nature 183:430

Van Allen JA, Ludwig GH, Ray EC, McIlwain CE (1958) Observations of high intensity radiation by satellites 1958 Alpha and Gamma. Jet Propul 28:588-592

Vandas M, Romashets EP, Watari S, Geranios A, Antoniadou E, Zacharopoulou O (2006) Camparison of force-freeflux rope model with observations of magnetic cloud. Adv Space Res 38:441-446

Vashenyuk EV, Balabin YV, Gvozdevsky BB (2009) Characteristics of relativistic solar cosmic rays from GLE modeling studies. In: Proceedings of 31st ICRC, Lodz, paper icrc1304

Vassiliadis D (2007) Forecasting space weather. In: Bothmer V, Daglis IA (eds) Space weather: physics and effects. Springer, Praxis

Vasyliunas VM (1975) Theoretical models of magnetic field line merging. Rev Geophys Space Phys 13:303-336

Velinov PIY, Mishev A (2007) The induced ionization by solar cosmic rays in the earth atmosphereCORSIKA code simulations. C R Acad Bulg Sci 60:493-500

Verma PL, Tiwari RK, Kumar Y, Nigam SK, Sharma AB, Khare N (2009) Halo coronal mass ejections: the cause of large Forbush decreases and Geomagnetic storms. In: Proceedings of 31st ICRC, Lodz, paper icrc0198

Viljanen A, Pirjola R (1994) Geomagnetically induced currents in the Finnish-high voltage power system. Surv Geophys 15:383

Viljanen A, Pulkkinen A, Pirjola R, Pajunpää K, Posio P, Koistinen A (2006) Recordings of geomagnetically induced currents and a nowcasting service of the Finnish natural gas pipeline system. Space Weather 4:S10004. doi:10.1029/2006SW000234

Von Baeckman, Schwenk W, Prinz W (1997) Handbook of Cathodic corrosion protection, 3rd edn. Gulf Publishing Co., Houston

Wagner WJ (1984) Coronal mass ejections. Annu Rev Astron Astrophys 22:267-289

Wallerius A (1982) Solen Gav Sverige en Stromstot. Ny teknik Teknisk tidskrift 29:3 
Walt M (1994) Introduction to geomagnetically trapped radiation. Cambridge University Press, Cambridge, p 168

Watari S (1997) The effect of the high-speed stream following the corotating interaction region on the geomagnetic activities. Ann Geophys 15:662-670. doi:10.1007/s00585-997-0662-5

Watari S (2008) Forecasting solar cycle 24 using the relationship between cycle length and maximum sunspot number. Space Weather 6:S12003. doi:10.1029/2008SW000397

Watermann J, Wintoft P, Sanahuja B, Saiz E et al (2009a) Models of solar wind structures and their interaction with the earth's space environment. Space Sci Rev 147:233-270

Watermann J, Vainio R, Lilensten J, Belehaki A, Messeroti M (2009b) The state of space weather scientific modeling —an introduction. Space Sci Rev 147:111-120

Weaver JT (1994) Mathematical methods for geo-electromagnetic induction. Research Studies Press (Wiley), Taunton

Webb DF, Crooker NU, Plunkett SP, St Cyr OC (2001) SEPs: Space weather hazard in interplanetary space. In: Song P et al (eds) Space weather, vol 125, Geophysical Monograph. American Geophysical Union, Washington, pp 123-141

Webb DF, Mizuno DR, Buffington A et al (2006) Solar mass ejection imager (SMEI) observations of coronal mass ejections (CMEs) in the heliosphere. J Geophys Res 111:A12101. doi:10.1029/2006 JA011655

Webb DF, Howard TA, Fry CD, Kuchar TA, Mizuno DR, Johnston JC, Jackson BV (2009) Studying geoeffective interplanetary coronal mass ejections between the sun and earth: space weather implications of solar mass ejection imager observations. Space Weather 7:S05002. doi:10.1029/2008 SW000409

Weigel RS, Detman T, Rigler EJ, Baker DN (2006) Decision theory and the analysis of rare event space weather forecasts. Space Weather 4:S05002. doi:10.1029/2005SW000157

Wellenhof BH, Lichtenegger H, Collins J (2001) GPS theory and practice, 5th edn. Springer, ISBN 3-21183534-2

White RS (1973) High-energy proton radiation belt. Rev Geophys 11:595-632

Wik M, Viljanen A, Pirjola R, Pulkkinen A, Wintoft P, Lundstedt H (2008) Calculation of geomagnetically induced currents in the $400 \mathrm{kV}$ power grids in southern Sweden. Space Weather 6:S07005. doi: $10.1029 / 2007$ SW000343

Williams DJ, Roelof EC, Mitchell DG (1992) Global magnetospheric imaging. Rev Geophys 30:183-208

Wilson JW et al (eds) (1997) Shielding strategies for human space exploration: a workshop. Johnson Space Center, Houston, 6-8 Dec 1995

Wilson JW, Kim MHY, Shinn JL, Tai H, Cucinotta FA, Badhwar GD, Badavi FF, Atwell W (1999) Solar cycle variation and application to the space radiation environment, NASA/TP-1999-209369

Yashiro S, Gopalswamy N, Michalek G, St Cyr OC, Plunkett SP, Rich NB, Howard RA (2004) A catalog of white light coronal mass ejections observed by the SOHO spacecraft. J Geophys Res 109:A07105. doi: 10.1029/2003JA010282

Zong QG, Fritz TA, Wilken B, Daly PW (2002) Energetic ions in the high latitude boundary layer of the magnetosphere-RAPID/cluster observation. In: Newell PT, Onsager TG (eds) The lower-latitude boundary layer. American Geophysical Union, Washington, pp 101-110

Zurbuchen TH, Richardson IG (2006) In-situ solar wind and magnetic field signatures of interplanetary coronal mass ejections. Space Sci Rev 123:31-43 